Cochrane Database of Systematic Reviews

\title{
Motor neuroprosthesis for promoting recovery of function after stroke (Review)
}

Mendes LA, Lima INDF, Souza T, do Nascimento GC, Resqueti VR, Fregonezi GAF

Mendes LA, Lima INDF, Souza T, do Nascimento GC, Resqueti VR, Fregonezi GAF.

Motor neuroprosthesis for promoting recovery of function after stroke.

Cochrane Database of Systematic Reviews 2020, Issue 1. Art. No.: CD012991.

DOI: 10.1002/14651858.CD012991.pub2.

www.cochranelibrary.com 
TABLE OF CONTENTS

HEADER

ABSTRACT

PLAIN LANGUAGE SUMMARY

SUMMARY OF FINDINGS

BACKGROUND

OBJECTIVES

METHODS

RESULTS

Figure 1.

Figure 2.

Figure 3.

DISCUSSION

AUTHORS' CONCLUSIONS

ACKNOWLEDGEMENTS

REFERENCES

CHARACTERISTICS OF STUDIES

DATA AND ANALYSES

Analysis 1.1. Comparison 1 Motor neuroprosthesis versus another assistive technology device, Outcome 1 Activities involving limbs: walking speed until 6 months of device use.

Analysis 1.2. Comparison 1 Motor neuroprosthesis versus another assistive technology device, Outcome 2 Activities involving limbs: walking speed between 6 and 12 months of device use.

Analysis 1.3. Comparison 1 Motor neuroprosthesis versus another assistive technology device, Outcome 3 Activities involving limbs: walking speed.

Analysis 1.4. Comparison 1 Motor neuroprosthesis versus another assistive technology device, Outcome 4 Activities involving limbs: TUG.

Analysis 1.5. Comparison 1 Motor neuroprosthesis versus another assistive technology device, Outcome 5 Activities involving limbs: mEFAP.

Analysis 1.6. Comparison 1 Motor neuroprosthesis versus another assistive technology device, Outcome 6 Participation scale of HRQoL.

Analysis 1.7. Comparison 1 Motor neuroprosthesis versus another assistive technology device, Outcome 7 Exercise capacity: 6MWT.

Analysis 1.8. Comparison 1 Motor neuroprosthesis versus another assistive technology device, Outcome 8 Balance: BBS. ......

Analysis 1.9. Comparison 1 Motor neuroprosthesis versus another assistive technology device, Outcome 9 Adverse events: number of dropouts during the intervention period.

Analysis 1.10. Comparison 1 Motor neuroprosthesis versus another assistive technology device, Outcome 10 Adverse events: serious adverse events related to intervention/during the intervention period.

Analysis 1.11. Comparison 1 Motor neuroprosthesis versus another assistive technology device, Outcome 11 Adverse events: falls.

ADDITIONAL TABLES

APPENDICES

CONTRIBUTIONS OF AUTHORS

DECLARATIONS OF INTEREST

SOURCES OF SUPPORT

DIFFERENCES BETWEEN PROTOCOL AND REVIEW

INDEX TERMS

1

1

2

4

7

8

8

11

12

14

15

18

20

20

21

29

47 
[Intervention Review]

\section{Motor neuroprosthesis for promoting recovery of function after stroke}

Luciana A Mendes ${ }^{1}$, Illia NDF Lima² ${ }^{2}$ Tulio Souza 3 , George C do Nascimentoㄴ, Vanessa R Resqueti ${ }^{5}$, Guilherme AF Fregonezi ${ }^{6}$

1PneumoCardioVascular Lab, Onofre Lopes University Hospital, Brazilian Company of Hospital Services (EBSERH) \& Department of Biomedical Engineering, Federal University of Rio Grande do Norte, Natal, Brazil. 2Faculty of Health Sciences of Trairi, Federal University of Rio Grande do Norte, Santa Cruz, Brazil. ${ }^{3}$ Department of Physical Therapy, Federal University of Rio Grande do Norte, Natal, Brazil. ${ }^{4}$ Department of Biomedical Engineering, Federal University of Rio Grande do Norte, Natal, Brazil. 5 PneumoCardioVascular Lab, Onofre Lopes University Hospital, Brazilian Company of Hospital Services (EBSERH) \& Department of Physical Therapy, Federal University of Rio Grande do Norte, Natal, Brazil. ${ }^{6}$ PneumoCardioVascular Lab, Onofre Lopes University Hospital, Brazilian Company of Hospital Services (EBSERH), Natal, Brazil

Contact address: Luciana A Mendes, PneumoCardioVascular Lab, Onofre Lopes University Hospital, Brazilian Company of Hospital Services (EBSERH) \& Department of Biomedical Engineering, Federal University of Rio Grande do Norte, Natal, Rio Grande do Norte, 59078-970, Brazil.mendes.luciana.a@gmail.com.

Editorial group: Cochrane Stroke Group.

Publication status and date: New, published in Issue 1, 2020.

Citation: Mendes LA, Lima INDF, Souza T, do Nascimento GC, Resqueti VR, Fregonezi GAF. Motor neuroprosthesis for promoting recovery of function after stroke. Cochrane Database of Systematic Reviews 2020, Issue 1. Art. No.: CD012991. DOI: 10.1002/14651858.CD012991.pub2.

Copyright @ 2020 The Cochrane Collaboration. Published by John Wiley \& Sons, Ltd.

\section{A B S T R A C T}

\section{Background}

Motor neuroprosthesis (MN) involves electrical stimulation of neural structures by miniaturized devices to allow the performance of tasks in the natural environment in which people live (home and community context), as an orthosis. In this way, daily use of these devices could act as an environmental facilitator for increasing the activities and participation of people with stroke.

\section{Objectives}

To assess the effects of MN for improving independence in activities of daily living (ADL), activities involving limbs, participation scales of health-related quality of life (HRQoL), exercise capacity, balance, and adverse events in people after stroke.

\section{Search methods}

We searched the Cochrane Stroke Group Trials Register (searched 19 August 2019), the Cochrane Central Register of Controlled Trials (CENTRAL) (August 2019), MEDLINE (1946 to 16 August 2019), Embase (1980 to 19 August 2019), and five additional databases. We also searched trial registries, databases, and websites to identify additional relevant published, unpublished, and ongoing trials.

\section{Selection criteria}

Randomized controlled trials (RCTs) and randomized controlled cross-over trials comparing MN for improving activities and participation versus other assistive technology device or MN without electrical stimulus (stimulator is turned off), or no treatment, for people after stroke.

\section{Data collection and analysis}

Two review authors independently selected trials, extracted data, and assessed risk of bias of the included studies. Any disagreements were resolved through discussion with a third review author. We contacted trialists for additional information when necessary and performed all analyses using Review Manager 5. We used GRADE to assess the certainty of the evidence. 


\section{Main results}

We included four RCTs involving a total of 831 participants who were more than three months poststroke. All RCTs were of MN that applied electrical stimuli to the peroneal nerve. All studies included conditioning protocols to adapt participants to MN use, after which participants used MN from up to eight hours per day to all-day use for ambulation in daily activities performed in the home or community context. All studies compared the use of MN versus another assistive device (ankle-foot orthosis [AFO]). There was a high risk of bias for at least one assessed domain in three of the four included studies.

No studies reported outcomes related to independence in ADL. There was low-certainty evidence that AFO was more beneficial than MN on activities involving limbs such as walking speed until six months of device use (mean difference (MD) $-0.05 \mathrm{~m} / \mathrm{s}, 95 \%$ confidence interval (CI) -0.10 to $-0.00 ; \mathrm{P}=0.03 ; 605$ participants; 2 studies; $1^{2}=0 \%$; low-certainty evidence); however, this difference was no longer present in our sensitivity analysis (MD $-0.07 \mathrm{~m} / \mathrm{s}, 95 \% \mathrm{Cl}-0.16$ to $0.02 ; \mathrm{P}=0.13 ; 110$ participants; 1 study; $12=0 \%)$. There was low to moderate certainty that $\mathrm{MN}$ was no more beneficial than AFO on activities involving limbs such as walking speed between 6 and 12 months of device use (MD 0.00 $\mathrm{m} / \mathrm{s}, 95 \% \mathrm{Cl}-0.05$ to $0.05 ; \mathrm{P}=0.93 ; 713$ participants; 3 studies; $\mathrm{I}^{2}=17 \%$; low-certainty evidence), Timed Up and Go (MD $0.51 \mathrm{~s}, 95 \% \mathrm{Cl}-4.41$ to 5.43; $\mathrm{P}=0.84 ; 692$ participants; 2 studies; $12=0 \%$; moderate-certainty evidence), and modified Emory Functional Ambulation Profile (MD 14.77 s, $95 \% \mathrm{Cl}-12.52$ to $42.06 ; \mathrm{P}=0.29 ; 605$ participants; 2 studies; $12=0 \%$; low-certainty evidence). There was no significant difference in walking speed when MN was delivered with surface or implantable electrodes (test for subgroup differences $\mathrm{P}=0.09 ; \mathrm{I}^{2}=65.1 \%$ ).

For our secondary outcomes, there was very low to moderate certainty that MN was no more beneficial than another assistive device for participation scales of $\mathrm{HRQ} \mathrm{oL}$ (standardized mean difference $0.26,95 \% \mathrm{Cl}-0.22$ to $0.74 ; \mathrm{P}=0.28 ; 632$ participants; 3 studies; $\mathrm{I}^{2}=77 \%$; very low-certainty evidence), exercise capacity (MD $-9.03 \mathrm{~m}, 95 \% \mathrm{Cl}-26.87$ to $8.81 ; \mathrm{P}=0.32 ; 692$ participants; 2 studies; $\mathrm{I}^{2}=0 \%$; lowcertainty evidence), and balance ( $\mathrm{MD}-0.34,95 \% \mathrm{Cl}-1.96$ to $1.28 ; \mathrm{P}=0.68 ; 692$ participants; 2 studies; $\mathrm{I}=0 \%$; moderate-certainty evidence). Although there was low- to moderate-certainty evidence that the use of $\mathrm{MN}$ did not increase the number of serious adverse events related to intervention (risk ratio (RR) $0.35,95 \% \mathrm{Cl} 0.04$ to 3.33; $\mathrm{P}=0.36 ; 692$ participants; 2 studies; $12=0 \%$; low-certainty evidence) or number of falls (RR 1.20, 95\% Cl 0.92 to 1.55; $\mathrm{P}=0.08 ; 802$ participants; 3 studies; $\mathrm{I}^{2}=33 \%$; moderate-certainty evidence), there was low-certainty evidence that the use of $\mathrm{MN}$ in people after stroke may increase the risk of participants dropping out during the intervention (RR $1.48,95 \%$ $\mathrm{Cl} 1.11$ to $1.97 ; \mathrm{P}=0.007 ; 829$ participants; 4 studies; $\mathrm{I}^{2}=0 \%$ ).

\section{Authors' conclusions}

Current evidence indicates that MN is no more beneficial than another assistive technology device for improving activities involving limbs measured by Timed Up and Go, balance (moderate-certainty evidence), activities involving limbs measured by walking speed and modified Emory Functional Ambulation Profile, exercise capacity (low-certainty evidence), and participation scale of HRQoL (very low-certainty evidence). Evidence was insufficient to estimate the effect of MN on independence in ADL. In comparison to other assistive devices, MN does not appear to increase the number of falls (moderate-certainty evidence) or serious adverse events (low-certainty evidence), but may result in a higher number of dropouts during intervention period (low-certainty evidence).

\section{PLAIN LANGUAGE SUMMARY}

\section{Motor neuroprosthesis for improving activities and participation of people in their natural environment after stroke}

\section{Review question}

Is motor neuroprosthesis $(\mathrm{MN})$ effective for improving activities and participation of people in their natural environment after stroke?

\section{Background}

Stroke survivors usually face long-term impairment, activity limitation, and reduced participation. MN consists of electronic devices that electrically stimulate a nervous system structure to help the performance of daily activities in the natural environment in which people live, as an orthosis (a device applied to a body segment to optimize position, or to limit or assist movement). However, the role of MN for improving activities and participation after stroke is unclear.

\section{Study characteristics}

We found four studies of MN involving a total of 831 participants who more than three months poststroke, with mean ages from 53 to 64 years. All participants were able to walk from less than $0.5 \mathrm{~m} / \mathrm{s}$ to more than 0.7 or even $0.9 \mathrm{~m} / \mathrm{s}$. The included studies were published between 2007 and 2015 in the USA and the Netherlands. All included studies applied MN directed to a nerve in the leg (peroneal nerve) to promote the contraction of a muscle at the front of the leg, thus preventing the foot 'dropping' as the leg was swung forward while the participant walked. MN was used from up to eight hours per day to all-day use for walking about in the natural environment in which people live. Three studies used an MN device that interfaces with the nervous system through electrodes positioned over the skin in the projection of the peroneal nerve in the leg. Only one study used a implantable device whose electrical stimulus is released directly on the nerve by electrodes placed under the layer that surrounds the nerve. All studies compared MN versus ankle-foot orthosis (AFO), that is an assistive device usually made of a rigid material and placed externally on the lower leg to hold the foot and ankle to prevent the foot dropping. 


\section{Key results}

There is limited evidence that people after stroke who receive $\mathrm{MN}$ as an orthosis for walking in the home or community context may not improve activities involving limbs such as walking speed between 6 and 12 months of device use (low-certainty evidence), Timed Up and Go (moderate-certainty evidence), and modified Emory Functional Ambulation Profile (low-certainty evidence); as well as participation scale of health-related quality of life (very low-certainty evidence), exercise capacity (low-certainty evidence), and balance (moderate-certainty evidence), compared with people after stroke who receive AFO. There was evidence of an effect that the control intervention (AFO) attained a higher walking speed after six months of device use (low-certainty evidence), but this evidence showed that the improvements were too small to indicate a meaningful change to patients, and when we excluded the study in which the people that assessed the outcomes were aware of the intervention details, this effect was no longer found. There was no difference in effects on walking speed between MN with surface versus MN with implantable electrodes. No study reported outcomes related to independence in activities of daily living.

The majority of studies reported adverse events such as falls and serious adverse events related to device use, which were found to be similar for MN and AFO use (moderate- and low-certainty evidence, respectively). One study considered serious adverse events related to device use as serious falls. More people who received MN withdrew from the studies than did people who received AFO (low-certainty evidence). The results of this review indicate that little is known about the effects of $\mathrm{MN}$ and that further information is required.

It is unknown if people less than three months poststroke could benefit from MN use as an assistive device to perform activities in daily life. The impact of MN applied to the upper limb or MN that uses brain or muscle signals to trigger the stimulation is unknown in people with stroke. We found no evidence evaluating the costs of delivering MN.

\section{Certainty of the evidence}

The certainty of the evidence ranged from moderate to very low. 


\begin{tabular}{|c|c|c|c|c|c|c|c|}
\hline \multicolumn{8}{|c|}{$\begin{array}{l}\text { S U M M A R Y O F F I N D I N G S } \\
\text { Summary of findings for the main comparison. } \\
\text { function after stroke }\end{array}$} \\
\hline \multicolumn{8}{|c|}{ Motor neuroprosthesis compared to another assistive technology device for promoting recovery of function after stroke } \\
\hline \multicolumn{8}{|c|}{$\begin{array}{l}\text { Patient or population: promoting recovery of function after stroke } \\
\text { Setting: home or community context } \\
\text { Intervention: motor neuroprosthesis } \\
\text { Comparison: another assistive technology device }\end{array}$} \\
\hline \multirow{2}{*}{\multicolumn{2}{|c|}{ Outcomes }} & \multicolumn{2}{|c|}{ Anticipated absolute effects* $(95 \% \mathrm{Cl})$} & \multirow{2}{*}{$\begin{array}{l}\text { Relative ef- } \\
\text { fect } \\
(95 \% \mathrm{CI})\end{array}$} & \multirow{2}{*}{$\begin{array}{l}\text { Number of } \\
\text { participants } \\
\text { (studies) }\end{array}$} & \multirow{2}{*}{$\begin{array}{l}\text { Certainty of } \\
\text { the evidence } \\
\text { (GRADE) }\end{array}$} & \multirow[t]{2}{*}{ Comments } \\
\hline & & $\begin{array}{l}\text { Risk with another as- } \\
\text { sistive technology de- } \\
\text { vice }\end{array}$ & $\begin{array}{l}\text { Risk with motor neuro- } \\
\text { prosthesis }\end{array}$ & & & & \\
\hline \multicolumn{2}{|c|}{$\begin{array}{l}\text { Independence in activities of daily } \\
\text { living }\end{array}$} & (No data) & - & - & No studies & $\begin{array}{l}\text { Insufficient } \\
\text { evidence }\end{array}$ & $\begin{array}{l}\text { No trials measured this } \\
\text { outcome. }\end{array}$ \\
\hline \multirow[t]{3}{*}{$\begin{array}{l}\text { Activities in- } \\
\text { volving limbs }\end{array}$} & $\begin{array}{l}\text { Walking speed un- } \\
\text { til } 6 \text { months of de- } \\
\text { vice use }(\mathbf{m} / \mathbf{s}) \\
\text { timed measures } \\
\text { at the end of treat- } \\
\text { ment }\end{array}$ & $\begin{array}{l}\text { The mean walking } \\
\text { speed in the control } \\
\text { group was on average } \\
\mathbf{0 . 5 8} \mathbf{~ m / s .}\end{array}$ & $\begin{array}{l}\mathbf{0 . 0 5} \text { mean difference } \\
\text { lower } \\
(0.1 \text { lower to } 0) \text { on inter- } \\
\text { vention group }\end{array}$ & - & 605 & $\begin{array}{l}\oplus \oplus \oplus \ominus \\
\text { Low }^{\mathrm{a}, \mathrm{b}}\end{array}$ & $\begin{array}{l}\text { Minimal important dif- } \\
\text { ference for comfort- } \\
\text { able walking speed } \\
\text { in chronic stroke par- } \\
\text { ticipant is } 0.2 \mathrm{~m} / \mathrm{s} \\
\text { (Hiengkaew } 2012 \text { ). }\end{array}$ \\
\hline & $\begin{array}{l}\text { Walking speed be- } \\
\text { tween } 6 \text { and } 12 \\
\text { months of device } \\
\text { use }(\mathbf{m} / \mathbf{s}) \\
\text { timed measures } \\
\text { at the end of treat- } \\
\text { ment }\end{array}$ & $\begin{array}{l}\text { The mean walking } \\
\text { speed in the control } \\
\text { group was on average } \\
\mathbf{0 . 6 9} \mathbf{~ m / s .}\end{array}$ & $\begin{array}{l}\text { O mean difference } \\
\text { (0.05 lower to } 0.05 \text { higher) }\end{array}$ & - & $\begin{array}{l}713 \\
\text { (3 RCTs) }\end{array}$ & $\begin{array}{l}\oplus \oplus \ominus \ominus \\
\text { Low }^{\mathrm{a}, \mathrm{c}}\end{array}$ & $\begin{array}{l}\text { Minimal important dif- } \\
\text { ference for comfort- } \\
\text { able walking speed } \\
\text { in chronic stroke par- } \\
\text { ticipant is } 0.2 \mathrm{~m} / \mathrm{s} \\
\text { (Hiengkaew } 2012 \text { ). }\end{array}$ \\
\hline & $\begin{array}{l}\text { TUG (s) } \\
\text { timed measures } \\
\text { at the end of treat- } \\
\text { ment }\end{array}$ & $\begin{array}{l}\text { The mean TUG in the } \\
\text { control group was on } \\
\text { average } \\
\mathbf{2 7 . 5 7} \mathrm{s} \text {. }\end{array}$ & $\begin{array}{l}\mathbf{0 . 5 1} \text { mean difference } \\
\text { higher } \\
\text { ( } 4.41 \text { lower to } 5.43 \text { higher) } \\
\text { on intervention group }\end{array}$ & - & $\begin{array}{l}692 \\
\text { (2 RCTs) }\end{array}$ & $\begin{array}{l}\oplus \oplus \oplus \ominus \\
\text { Moderate }^{a}\end{array}$ & \\
\hline
\end{tabular}




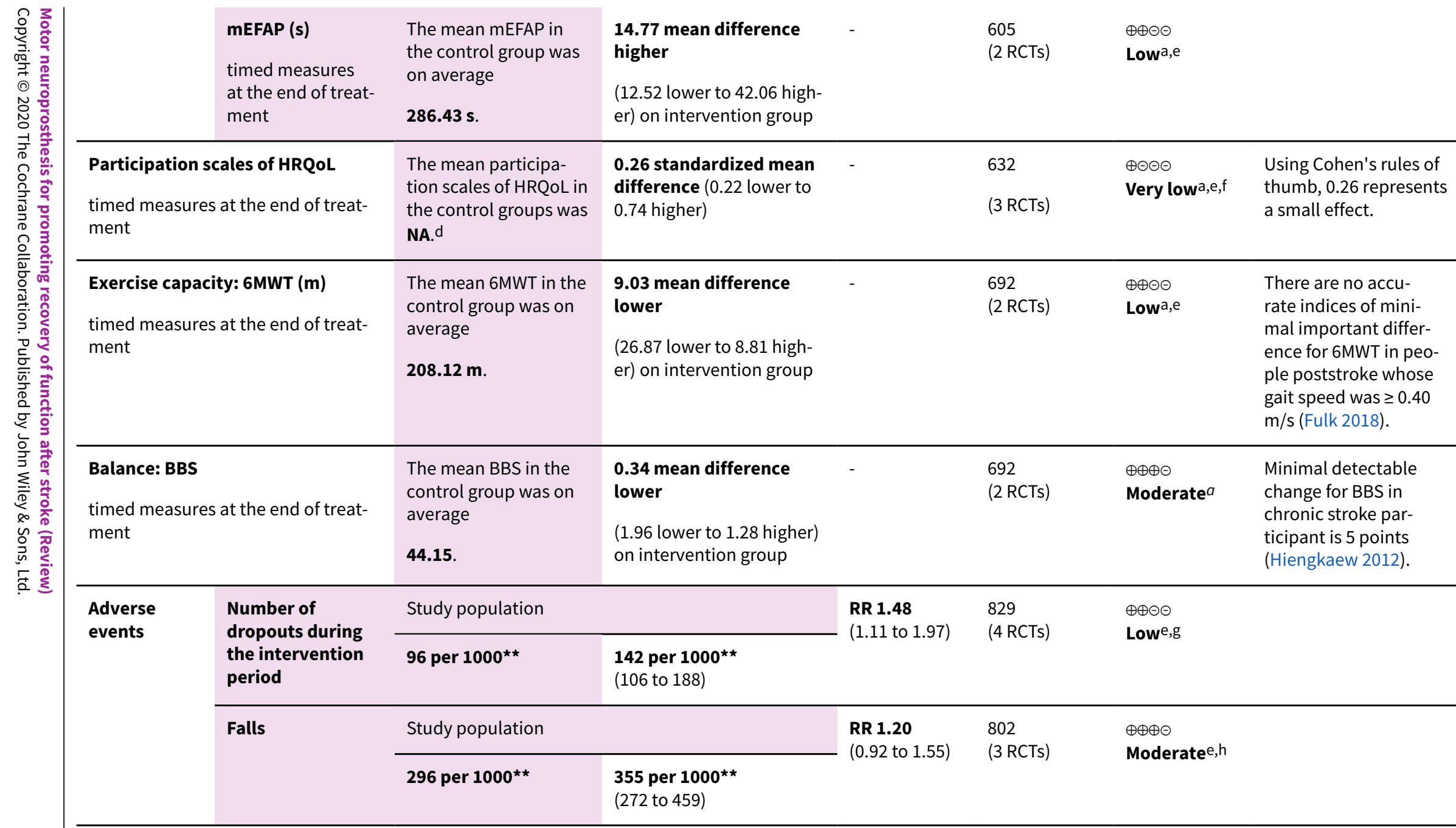

${ }^{*}$ The risk in the intervention group (and its $95 \%$ confidence interval) is based on the assumed risk in the comparison group and the relative effect of the intervention (and its $95 \% \mathrm{Cl}$ ).

**We used the median control group risk across studies.

6MWT: 6-minute walk test; BBS: Berg Balance Scale; Cl: confidence interval; HRQoL: health-related quality of life; mEFAP: modified Emory Functional Ambulation Profile; NA: not applicable; RCT: randomized controlled trial; RR: risk ratio; TUG: Timed Up and Go test.

GRADE Working Group grades of evidence

High certainty: We are very confident that the true effect lies close to that of the estimate of the effect. 
(he are moderately confident in the effect estimate: the true effect is likely to be close to the estimate of the effect, but there is a possibility that it is substantially different.

Low certainty: Our confidence in the effect estimate is limited: the true effect may be substantially different from the estimate of the effect.

Very low certainty: We have very little confidence in the effect estimate: the true effect is likely to be substantially different from the estimate of effect.

aThe outcome assessors were not blinded in the larger study.

The evidence of this effect is removed when sensitivity analysis is performed, suggesting some inconsistency in this finding.

cOne study has high risk of bias for incomplete outcome data.

dNo data can be provided due to the combination of different outcome measures for the same outcome in this analysis.

elmprecise due to confidence intervals that included potential for important harm or benefit.

${ }^{f}$ Considerable heterogeneity between trials.

gAs no study included motor neuroprosthesis (MN) directed to the upper limb, this effect addresses only lower limb MN and not the whole category of MN.

hAlthough two of the three studies were sponsored by the manufacturers, they clearly described fall events. There is no blinding of outcome assessment for the largest study, but this would seem not to interfere with this outcome. 


\section{B A C K G R O U N D}

\section{Description of the condition}

Among the cardiovascular diseases, hemorrhagic and ischemic strokes were considered to be the second and third most common causes of disability-adjusted life-years, respectively, in 2015 (Roth 2017). They present a higher prevalence among individuals aged 74 to 79 years (Roth 2017). Projections indicate that by 2030 there will be 70 million stroke survivors worldwide (Feigin 2014). The stroke survivors will face long-term impairment, activity limitation, and reduced participation that will impact not only on their own lives, but also on the lives of their families (Langhorne 2009). Among them, approximately one-third will have functional dependence during the first year after stroke (de Campos 2017). One of the important factors that contributes to being unable to live independently is motor impairment by hemiparesis, because it leads to difficulties in performing functional activities (Schiemanck 2006). Lower limb impairment typically affects the performance of gait, and it is common to observe foot drop when the individual tries to take a step with the paretic limb (Stein 2008). Upper limb impairment affects the interaction with objects in the environment, involving movements such as grasp, grip, pinch, and others (Lang 2013). In this scenario, the use of contextual factors, such as assistive technology devices (e.g. orthosis), work as a resource to facilitate the performance of daily activities and the recovery of motor function after stroke (Eng 2007).

\section{Description of the intervention}

The first application of electric current to nervous tissue in order to promote movement dates back to the experiment of Galvani in the 1790s (Cambridge 1977). Since then, there have been advances in the use of electrical stimulation of motor neurons to activate paralyzed or paretic muscles, and it is widely used in clinical rehabilitation (Sheffler 2007). This electrical stimulus applied to excite peripheral sensory and motor nerves is known as neuromuscular electrical stimulation (NMES) (Alon 2003b); when the aim is to employ this stimulus to achieve functional tasks, the term used is functional electrical stimulation (FES) (Sheffler 2007). FES is a routine therapeutic approach that physiotherapists use during stroke rehabilitation in a clinical setting to improve strength, upper extremity function, and gait, and to prevent hemiplegic shoulder subluxation (Auchstaetter 2016).

Due to technological advances, especially in electronics, electrical stimulation devices have become increasingly miniaturized and lightweight, and with more refined control and sensor configurations, they can be worn as an orthosis beyond the clinical setting (Melo 2015; Popović 2014). By integrating the electric stimulator with control algorithms and sensors, it is possible to determine the time of delivery of the electrical current in response to the sensor signals (Melo 2015). This integration was implemented for the first time in 1961 when Liberson applied electrical stimuli to the common peroneal nerve to activate the tibialis anterior muscle during the swing phase of gait. He used a heel switch as a sensor to control the timing of the stimulation. The train of stimuli was only released when the heel came off the ground at the end of the stance phase and ended when the heel contacted the ground again at the beginning of the stance phase (Liberson 1961). Since then, much progress has been made, with devices becoming portable, battery powered, and wireless, allowing them to be worn and implemented as an assistive technology device (e.g. an orthosis) that acts as an environmental facilitator for expanded capacity and performance in walking and moving and also carrying and handling objects (Bosch 2014; Cowan 2012). In addition to this direct effect on performance, the orthotic use of the electric current enables people with stroke to experience a greater amount of practice in their current environment. This orthotic use is often referred to as motor neuroprosthesis (MN), which is considered to be an electronic device that interfaces with the nervous system and attempts to restore functions, generally by electrical stimulation (Naik 2014; Ziat 2015).

The activation of neural structures to promote movement through electrical stimulation is used in both MN and FES, meaning there may be overlap between FES and MN concepts (Popović 2014). Although both MN and FES use electrical stimulation, MN has a system technology configuration that allows its use in the actual context in which people live (real-world setting). In this way MN allows the electrical stimulus to be used as an environmental facilitator (e.g. an orthosis) to achieve a greater level of practice, producing effects during the performance of functional abilities in the individual's current environment (Laufer 2009). Several studies and guidelines already consider comparisons of MN with other orthotic devices for decision-making purposes (Bethoux 2015; Bosch 2014; Kluding 2014; NICE 2009). We focused on this perspective within the scope of this review, that is that MN consists of a category that uses stimuli to allow the performance of tasks in the actual context in which a person lives, and is being used daily for increasing the activities and participation of people with stroke, while FES comprises the use of electrical stimulation to enhance function (Martin 2012; Sheffler 2007), and is especially used in the context of the clinical setting. Several Cochrane Reviews have already shown evidence of therapies for improving activities of daily living (ADL) such as virtual reality (Laver 2017), action observation (Borges 2018), and mirror therapy (Thieme 2018). Within the context of rehabilitation, these therapies may be additional and further enhanced by MN daily use.

In order to operate autonomously during the performance of functional activities, MN has a typical architecture composed of a network of sensors, control unit, and a stimulation unit (Melo 2015). The stimulation unit is responsible for generating the electric current that is delivered to the nervous system via electrodes placed in different locations, ranging from the skin surface to directly implanted into different areas of the nervous system (Collinger 2013). Regardless of the location of this interface in the nervous system, all devices that stimulate it electrically for the previously described purposes are considered to be MN. It is possible to use biological signals, such as electromyography, electroencephalography, and electroneurography signals, eye tracking, and voice control, or non-biological signals such as force/pressure and inertial sensors as an input to trigger the electrical stimulus to the desired motor function (Ambrosini 2014). Consequently, there is a need to translate and to adjust the command signal provided by sensors as an input to the stimulation unit, a function of the control unit (Horch 2004; Naik 2014). Besides the described requirements, the device needs to be portable, lightweight, autonomously controlled, and battery powered to be an assistive technology device (Melo 2015).

\section{How the intervention might work}

MN allows people with stroke to enhance the performance of functional activities in the home and community, including the 
manipulation of objects with the paretic upper limb or gait activities with the paretic lower limb (Cowan 2012; Moss 2011; Sheffler 2009). The use of these assistive devices can lead people with stroke to benefit from their orthotic effect, reflecting the direct improvement in tasks while using the MN (Dunning 2015; Kottink 2004; Prenton 2016). Furthermore, the daily use of MN allows people with stroke to perform repetitive activities that lead to a longer-lasting improvement (as an effect of relearning) after the stimulation is turned off (Ambrosini 2011; Dunning 2015; Prenton 2016). This may be explained by plasticity mechanisms from peripheral effects in muscles and central effects from the central nervous system reorganization. It is hypothesized that these devices activate the motor-related areas of the cortex and their residual corticospinal pathways induce neural plasticity in people with stroke (Everaert 2010). Thus far, direct signs of brain injury repair after one year of using the MN in people with chronic stroke were seen by cortical metabolism improvement over the damaged motor areas, leading to recovery of near-to-normal brain metabolism (Thibaut 2017).

\section{Why it is important to do this review}

Some systematic reviews have been conducted on the topic of FES that considered devices with the architecture configuration of MN to promote recovery of function after stroke (Bolton 2004; Dunning 2015; Kottink 2004; Meilink 2008). Only one of these reviews considered daily use of $\mathrm{MN}$ devices in the home or community context as an assistive device; however, this review only analyzed surface MN directed to a specific part of the lower limb, without performing a meta-analysis (Dunning 2015). In order to determine the level of evidence of the effects of the daily use of the whole category of upper limb and lower limb MN for improving activities and participation in the natural environment in which people with stroke live, it was essential to conduct this high-quality systematic review.

Due to the wide variety of $M N$, there is a need to clarify which device has the best evidence for improving activity and participation after stroke, the best phase in which to use the device, the optimal frequency of use, and which target shows the best results. Moreover, to support clinical practice, healthcare managers, policymakers, and consumers, and the acceptability of using MN, costs, and benefits, must be considered. This review aimed to synthesize the evidence for the use of MN for improving activities and participation after stroke and hence to assist clinical decision-making.

\section{O B JE C T IVES}

To assess the effects of motor neuroprosthesis (MN) for improving independence in activities of daily living (ADL), activities involving limbs, participation scales of health-related quality of life (HRQoL), exercise capacity, balance, and adverse events in people after stroke.

\section{METHO D S}

\section{Criteria for considering studies for this review}

\section{Types of studies}

We planned to review published and unpublished randomized controlled trials (RCTs) and randomized controlled cross-over trials. For randomized controlled cross-over trials, we only analyzed the first period as a parallel-group trial. Cross-over trials were only eligible if comparison groups included placebo; the evaluation of outcomes was blinded to allocation; and a minimum period of follow-up was clearly described. Trials reported in abstract form were eligible for inclusion only when adequate information was provided in the abstract or was available from the trial authors. We excluded quasi-RCTs, that is trials in which the method of allocating participants to a treatment is not strictly random (e.g. by date of birth, hospital record number, or alternation). If we included a study that was described as randomized, but while assessing risk of bias we learned that it was a quasi-RCT, we excluded the data from this study from the analysis.

\section{Types of participants}

We included studies whose participants were clinically diagnosed with stroke, were over 18 years of age, of both sexes, at any stage of the disease. A diagnosis of stroke fulfills the clinical criteria of the World Health Organization (WHO); stroke is defined as a "neurological deficit of cerebrovascular cause that lasts more than 24 hours or leads to death within 24 hours" (WHO 1989). A diagnosis of stroke encompasses ischemic and hemorrhagic stroke (including subarachnoid, intraventricular, or intracerebral hemorrhage).

\section{Types of interventions}

This review included studies that used motor neuroprosthesis (MN) devices for improving activities and participation after stroke. Considering that this approach focuses on the use of $\mathrm{MN}$ as an orthosis, we only included studies that used MN in the home or community context and that fulfilled some device requirements, such as working autonomously, being battery powered to ensure its autonomy, and have stimulus triggered by a sensor. We also included studies that used implanted or superficial electrodes whose application is directed to upper or lower limbs, and studies that addressed hybrid MN, which combines an exoskeleton or a mechanical orthosis with an electrical stimulation device. We excluded studies that used sensory stimulation as transcutaneous electrical nerve stimulation (TENS).

We selected studies that included the following comparisons.

- MN with electrical stimulus versus no treatment.

- MN with electrical stimulus versus MN without electrical stimulus, where both groups used the device, but in one group the stimulator was turned off.

- MN versus another assistive technology device (e.g. foot drop stimulator versus ankle foot orthosis, electromyographic (EMG)triggered stimulation versus hand orthosis, etc).

\section{Types of outcome measures}

We included outcome measures falling into the International Classification of Functioning, Disability and Health (ICF) categories for activity and participation (Brehm 2011; Mudge 2007; Sullivan 2013). According to the ICF, 'activity' corresponds to the execution of a task or action by an individual, while 'participation' means the involvement in a life situation (WHO 2001).

\section{Primary outcomes}

- Independence in ADL, e.g. Functional Independence Measure (FIM) (Hamilton 1994), Barthel Index (BI) (Quinn 2011), Motor Assessment Scale (MAS) (Dean 1992). 
- Activities involving limbs, e.g. Jebsen Taylor Hand Function Test (Stern 1992), Wolf Motor Function Test (WMFT) (Wolf 2001), 9Hole Peg Test (9HPT) (Heller 1987), Box \& Blocks Test (BBT) (Mathiowetz 1985), Motor Activity Log (MAL) (Uswatte 2005), Timed Up and Go test (TUG) (Podsiadlo 1991), Rivermead Mobility Index (Collen 1991), Functional Ambulation Categories (FAC) (Holden 1984), Dynamic Gait Index (Jonsdottir 2007), modified Emory Functional Ambulation Profile (mEFAP) (Baer 2001; Wolf 1979), walking speed.

\section{Secondary outcomes}

- Participation scales of HRQoL, e.g. 36-Item Short Form Health Survey (SF-36) (Anderson 1996), Stroke Impact Scale (SIS) (Duncan 1999).

- Exercise capacity, e.g. 6-minute walk test (6MWT) (Seale 2006).

- Balance, e.g. Berg Balance Scale (BBS) and Functional Reach Test (FRT) (Berg 1992; Martins 2012).

- Adverse events, i.e. pain, skin irritation, dropouts, acceptance, number of falls.

\section{Adverse events}

To measure the acceptance of MN we considered the number of withdrawals or dropouts from the study due to any reason during the study period. We used the incidence of serious adverse events related to intervention and number of falls to investigate the safety of $\mathrm{MN}$. We considered the number of falls due to the nature of the use of MN in the home or community context for walking activities.

\section{Search methods for identification of studies}

See the 'Specialized register' information at the Cochrane Stroke Group's website. We searched for trials in all languages and arranged for the translation of relevant articles when necessary.

\section{Electronic searches}

We searched the Cochrane Stroke Group Trials Register (last searched 19 August 2019) and the following electronic bibliographic databases.

- The Cochrane Central Register of Controlled Trials (CENTRAL) (Issue 8 of 12, August 2019) in the Cochrane Library (searched 19 August 2019; Appendix 1)

- MEDLINE Ovid (1946 to 16 August 2019; Appendix 2)

- Embase Ovid (1980 to 2019 Week 33; searched 19 August 2019; Appendix 3)

- CINAHL EBSCO (Cumulative Index to Nursing and Allied Health Literature; 1982 to 19 August 2019; Appendix 4)

- AMED Ovid (Allied and Complementary Medicine Database; 1985 to 19 August 2019; Appendix 5)

- PEDro (Physiotherapy Evidence Database; www.pedro.org.au/; searched 19 August 2019; Appendix 6)

- REHABDATA (www.naric.com/?q=en/REHABDATA; searched 19 August 2019; Appendix 7);

- IEEE (Institute of Electrical and Electronics Engineers; www.ieee.org/; searched 19 August 2019; Appendix 8)

We developed the MEDLINE search strategy with the help of the Cochrane Stroke Group Information Specialist and modified it for the other databases. The search strategy included Cochrane's highly sensitive search strategies for identification of RCTs, as described in the Cochrane Handbook for Systematic Reviews of Interventions (Lefebvre 2011), and the Cochrane Stroke Group's search strategies for the identification of stroke studies in respective databases and other resources.

We also searched the following electronic registries, databases, and websites to identify additional relevant published, unpublished, and ongoing trials.

- US National Institutes of Health Ongoing Trials Register ClinicalTrials.gov (www.clinicaltrials.gov; searched 19 August 2019; Appendix 9)

- World Health Organization (WHO) International Clinical Trials Registry Platform (www.who.int/ictrp/en/; searched 19 August 2019; Appendix 10)

- Stroke Trials Registry (www.strokecenter.org/trials/; searched 21 August 2019; Appendix 11)

- ISRCTN registry (www.isrctn.com/; searched 20 August 2019; Appendix 12)

- Australian New Zealand Clinical Trials Registry (www.anzctr.org.au/; searched 20 August 2019; Appendix 13)

- Health Technology Assessment (HTA) database - Centre for Reviews and Dissemination, University of York (www.crd.york.ac.uk/PanHTA; searched 19 August 2019; Appendix 14)

- OAlster (oaister.worldcat.org/; searched 19 August 2019; Appendix 15)

- The Directory of Open Access Repositories - OpenDOAR (searched using CORE; searched 19 August 2019; Appendix 16)

- British Library Ethos (ethos.bl.uk/; searched 19 August 2019; Appendix 17)

- ProQuest Dissertations \& Theses Global (www.proquest.com/ products-services/pqdtglobal.html; 19 August 2019; Appendix 18)

\section{Searching other resources}

We contacted equipment manufacturers to ask for information about any relevant trials that address MN (Appendix 19). We contacted some original study authors for clarification and further data if trial reports were unclear. Additionally, we used Grey Matters: a practical tool for searching health-related grey literature checklist, published by the Canadian Agency for Drugs and Technologies in Health (CADTH) to conduct additional searches of grey literature (Grey Matters; www.cadth.ca/resources/findingevidence/grey-matters; searched 19 August 2019; Appendix 20).

\section{Data collection and analysis}

\section{Selection of studies}

Two review authors (LM and IN) independently screened the titles and abstracts of the studies identified by the search and removed obviously irrelevant reports. We obtained the full-text of the remaining studies, and the same two review authors selected studies for inclusion according to the predefined inclusion criteria. In the case of any questions on methodology, we contacted the study authors for clarification to determine study eligibility. Two other review authors (VR or TS) evaluated any discrepancies as required and advised in case of disagreement. We recorded the reasons for exclusion and completed a PRISMA flow chart (Liberati 2009). 


\section{Data extraction and management}

Two review authors (LM and IN) independently extracted data from the included studies and summarized trial details on a data extraction template in Covidence that was developed specifically for this review (Covidence). In the case of incomplete or unclear data, we contacted the study authors for clarification. Any disagreements were resolved by discussion or by involving a third review author (VR or TS). We extracted the following information according to the methods described in the Cochrane Handbook for Systematic Reviews of Interventions (Higgins 2011a).

- General information: title of the review, study ID, and contact details.

- Methods: study design, instruments used, study duration, 'Risk of bias' criteria (sequence generation, allocation concealment, blinding, incomplete outcome data, selective outcome reporting), year of study.

- Participants: total number of participants, setting, age, sex, country, motor impairment, type of stroke, phase (acute, subacute, or chronic).

- Intervention: intervention details regarding time (number and duration of exposure, weeks of use, and in the case of follow-up, describe the duration), devices (type of electrode and sensor), and place of application (upper or lower limb); methods used in the control group.

- Outcomes: definition of primary and secondary outcome(s).

- Results: number of participants allocated to each group, number of withdrawals (by group, with reasons), adverse events, overall sample size and methods used to estimate statistical power (regarding the target number of participants to be recruited, the clinical difference to be detected, and the ability of the trial to detect this difference).

- Notes: contact with authors (information obtained or not), article in a language other than English, funding source and noteworthy conflicts of interest of study authors.

\section{Assessment of risk of bias in included studies}

Two review authors (LM and IN) independently assessed the risk of bias for each included study using the Cochrane 'Risk of bias' tool (Higgins 2011b). Any disagreements were resolved by discussion or by involving a third review author (VR or TS). We assessed risk of bias according to the following domains.

- Random sequence generation

- Allocation concealment

- Blinding of participants and personnel

- Blinding of outcome assessment

- Incomplete outcome data

- Selective outcome reporting

- Other bias

We graded the risk of bias for each domain as low, high, or unclear and entered this information along with the reasons for each decision into the 'Risk of bias' table produced for each study. We used Table 8.5.d in the Cochrane Handbook for Systematic Reviews of Interventions, which provides criteria for making judgements regarding risk of bias in each of the seven domains of the tool (Higgins 2011b). We considered the risk of bias of the studies and its contribution to the treatment effect. We then entered the data into Review Manager 5 (Review Manager 2014).

\section{Measures of treatment effect}

We performed the data analysis according to Cochrane guidelines. One review author (LM) entered the quantitative data into Review Manager 5 (Review Manager 2014), which was checked by another review author (IN), and analyzed. We presented the results for each outcome with 95\% confidence intervals (Cls). We measured treatment effects using the risk ratio (RR) for dichotomous outcomes, mean difference (MD) or standardized mean difference (SMD) (if different methods of measurement were used in the studies) and overall effect size (with $95 \% \mathrm{Cl}$ calculated) for continuous outcomes.

\section{Unit of analysis issues}

We considered the number of individual participants as the unit of analysis in this review. As we only identified individually randomized trials, we did not need to analyze for unit of analysis issues as planned in our protocol (Mendes 2018).

\section{Dealing with missing data}

When data were missing, we contacted the original researchers to request these data whenever possible. When this was not possible, and we considered that the missing data might introduce serious bias, we performed a sensitivity analysis to explore the impact of including such studies on the overall assessment of results. We assessed and reported dropout rates for each study, and used intention-to-treat (ITT) analyses (analysis of all participant data according to group allocation). We considered the amount of missing data when determining the risk of bias within included studies.

\section{Assessment of heterogeneity}

We assessed heterogeneity visually by observing the nonoverlapping of $\mathrm{Cl}$ in the forest plots. Once identified, we quantified statistical heterogeneity using the $1^{2}$ statistic. The $1^{2}$ statistic estimates the percentage of total variation across trials due to heterogeneity rather than to chance. We categorized heterogeneity as $1^{2}$ values of $40 \%$ or less as indicating heterogeneity might not be important; $30 \%$ to $60 \%$ may represent moderate heterogeneity; $50 \%$ to $90 \%$ may represent substantial heterogeneity; and $75 \%$ or above indicating considerable heterogeneity (Higgins 2011c).

\section{Assessment of reporting biases}

We intended to perform a funnel plot analysis to assess reporting bias if a sufficient number of studies was identified (i.e. 10 or more). Had asymmetry been present, we would have explored possible causes, including publication bias, poor methodological quality, and true heterogeneity.

\section{Data synthesis}

We planned to perform a random-effects meta-analysis and use the fixed-effect method as a sensitivity analysis.

\section{GRADE and 'Summary of findings' table}

We assessed the certainty of the evidence by creating a 'Summary of findings' table using the following outcomes: independence in ADL, activities involving limbs, participation, 
exercise capacity, balance, and adverse events. We used the five GRADE considerations (study limitations, consistency of effect, imprecision, indirectness, and publication bias) to assess the certainty of a body of evidence as it relates to the studies contributing data to the review for the outcomes (Atkins 2004). We used GRADEpro GDT to prepare the 'Summary of findings' table for the main comparison and to report the certainty of the evidence (GRADEpro GDT).

\section{Subgroup analysis and investigation of heterogeneity}

We planned to examine the following subgroup analyses if data were available.

- Type of effect (first subgroup defined as immediate effect or orthotic effect, i.e. the effect seen while using $M N$; second subgroup defined as relearn effect, i.e. the effect seen after the stimulation is turned off).

- Effect of MN when device was used for varying durations (4 weeks of use, between 5 and 24 weeks of use, 25 weeks of use).

- Effect of MN when used by participants at different phases of disease ( $<3$ months, 3 months).

- Effect of MN with surface or implantable electrodes.

- Effect of MN when applied on lower limb or upper limb.

We planned to use random-effects methods to produce this subgroup analysis for primary outcomes only.

\section{Sensitivity analysis}

We used Cochrane's tool for assessing risk of bias to judge the study methods (Higgins 2011b). We performed sensitivity analyses to assess the robustness of the findings by excluding studies from the analysis that were at high risk of bias in one or more of these three domains: random sequence generation, allocation concealment, and blinding of outcome assessors.

\section{RES U LTS}

\section{Description of studies}

See Characteristics of included studies; Characteristics of excluded studies; Characteristics of studies awaiting classification; Characteristics of ongoing studies.

\section{Results of the search}

Our electronic searches and searches of other resources identified a total of 18,579 references. After removal of 4881 duplicates, a total of 13,698 references remained for title and abstract screening. Of these, we excluded 13,616 references as irrelevant. We obtained the full text of 82 reports, and from these identified and included four studies (9 reports) in the review. The results of the search are summarized in the study flow diagram (Figure 1). 
Figure 1. Study flow diagram.

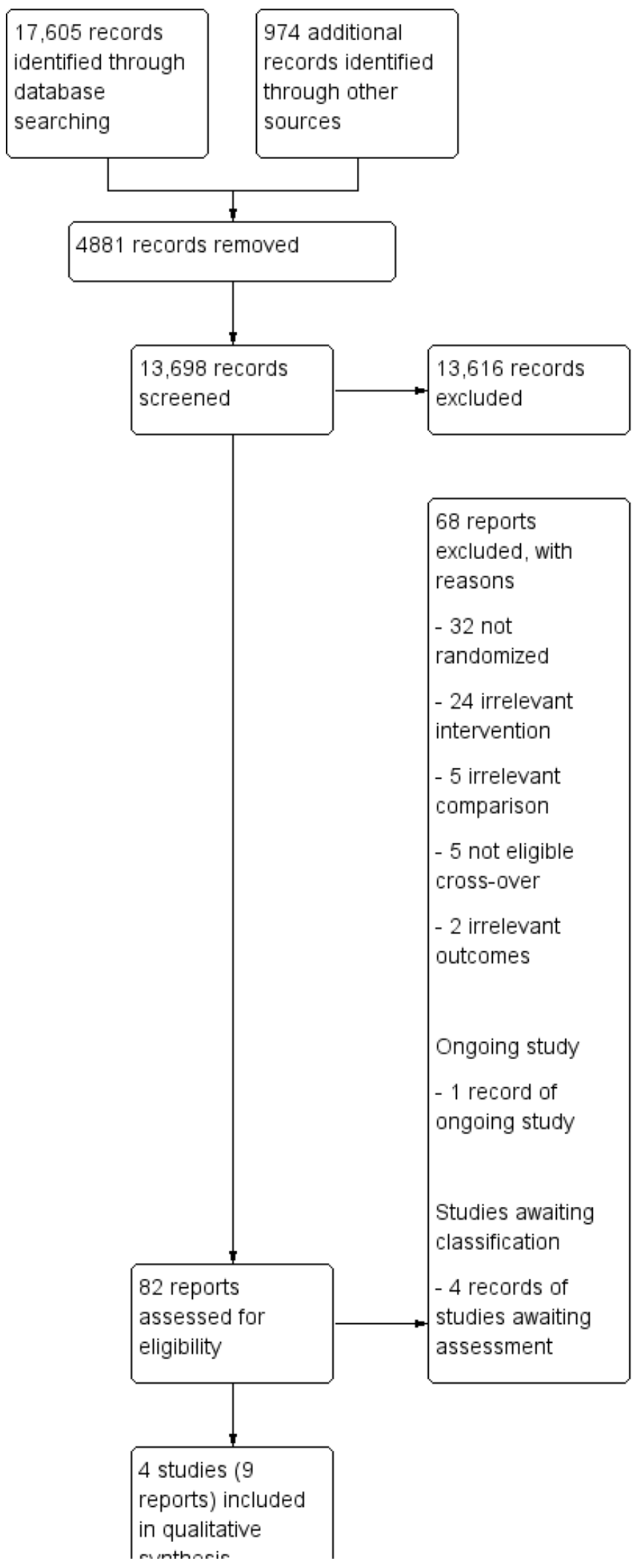


Figure 1. (Continued)

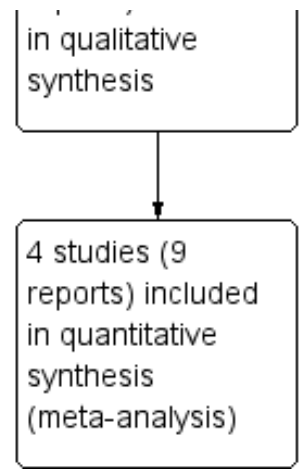

\section{Included studies}

See Characteristics of included studies.

\section{Sample size and study location}

The four included RCTs involved a total of 831 participants of both sexes, and were published between 2007 and 2015 in the USA, Bethoux 2014; Kluding 2013; Sheffler 2013a, and the Netherlands, Kottink 2007. Sample sizes ranged from 29 (14 and 15 in each group; Kottink 2007) to 497 (242 and 253 in each group; Bethoux 2014).

\section{Participant characteristics}

The mean age of participants ranged from 53, in Sheffler 2013a, to 64 years, in Bethoux 2014. Time poststroke varied between studies: Bethoux 2014 and Kottink 2007 recruited participants with a poststroke period of six months or more, whereas Kluding 2013 and Sheffler 2013a included participants with a poststroke period of three months or more. All participants were able to walk: in Sheffler 2013a they were able to walk at least 9.1 meters without an anklefoot orthosis; in Bethoux 2014 and Kluding 2013 they were able to walk at least 10 meters with or without an assistive device or with a maximum of one person assisting, respectively; and in Kottink 2007 participants needed to walk independently on level and nonlevel surfaces, stairs, and inclines. The mean walking speed varied among studies, from less than $0.5 \mathrm{~m} / \mathrm{s}$, Bethoux 2014; Kluding 2013; Sheffler 2013a, to more than 0.7 and 0.9 m/s, Kottink 2007 .

\section{Intervention approaches}

The included studies applied $\mathrm{MN}$ on a lower limb to facilitate walking in daily activities performed in the home or community context. All studies applied electrical stimuli to the peroneal nerve during the swing phase of gait using commercially available battery-powered devices. Three studies used a single-channel surface peroneal nerve stimulator composed of a stimulator, control unit, sensor, and two surface electrodes (Bethoux 2014; Kluding 2013; Sheffler 2013a), whereas one study used a twochannel implantable device composed of implantable components such as a stimulator, two leads, and two intraneural electrodes, and non-implantable components such as an external transmitter with a built-in antenna and sensor (Kottink 2007). Only one study used a tilt sensor and an accelerometer placed on the participant's leg to trigger the stimulation (Bethoux 2014); the other three studies used a heel switch placed inside the shoe to control the timing of the stimulation. Participants used MN from up to eight hours per day (Sheffler 2013a), to all-day use for ambulation (Bethoux 2014; Kluding 2013; Kottink 2007). During the first weeks of the intervention, all studies used conditioning protocols to adapt participants to MN use; after that, participants used MN for a long time during the day. All conditioning protocols included fitting the device to the participant's leg and giving the participant instructions on the use of MN (Table 1). The duration of the conditioning protocols ranged from two weeks, in Bethoux 2014, to six weeks, in Kluding 2013. These protocols were comprised of different activities, such as a progressive home-wear schedule to gradually increase time of device use, Bethoux 2014; Kluding 2013; Kottink 2007, and physical therapy sessions, which included gait training with the device, performed once or twice a week, Kluding 2013, and additional activities such as passive and active range-ofmotion exercises, lower extremity strengthening, standing balance, and weight-shifting activities to the affected limb (Sheffler 2013a). In Kottink 2007, participants underwent a surgical procedure for the implantation of the MN components, and stimulation during walking started in the third week after surgery. The total duration of exposure to interventions varied from 12 weeks, in Sheffler 2013a, to 12 months, in Bethoux 2014. All studies compared the use of MN versus another assistive device (ankle-foot orthosis [AFO]).

\section{Outcomes}

Outcome measures for each of the predefined outcome categories are shown in Table 2. No study included primary outcomes related to independence in ADL. As we listed walking speed as a primary outcome related to activities involving limbs, we considered measures that assess speed for a distance of 10 meters (the 10-meter walk test (10MWT)) and kinematic assessment with a motion analysis system (Watson 2002). All studies included comfortable walking speed as a primary outcome measured with the 10MWT, Bethoux 2014; Kluding 2013, or with a motion analysis system, Kottink 2007; Sheffler 2013a. We pooled comfortable walking speed data because both measures assessed speed with the same unit $(\mathrm{m} / \mathrm{s})$ and the same distance. Only one study assessed fast walking speed, also using the 10MWT (Kluding 2013). Other outcomes related to activities involving limbs were assessed with TUG, Bethoux 2014; Kluding 2013, and mEFAP, Bethoux 2014; Sheffler 2013a. We included the data for outcomes related to participation in the Stroke-Specific Quality of Life (SSQoL) (Williams 1999), reported in two studies (Bethoux 2014; Sheffler 2013a). It can therefore be said that all studies included outcomes related to participation, but the scale used varied among studies: SF-36 (Kottink 2007), SIS (Bethoux 2014; Kluding 2013), and the SSQoL previously mentioned. The total value of the quality of life scale was only presented in studies that reported SSQoL. One study reported the values of each domain of the scale SF-36 
separately as well as the Physical Component Summary (PCS-36) and Mental Component Summary (MCS-36) (Kottink 2007). Kluding 2013 reported the values of some domains of the SIS. Two studies assessed the exercise capacity-related outcome using the 6MWT (Bethoux 2014; Kluding 2013), and the balance-related outcomes using the BBS, Bethoux 2014; Kluding 2013, and FRT, Kluding 2013. Considering that the only study that described FRT also evaluated BBS, and that functional reach is an outcome assessed in the BBS as well, we decided to present only data for BBS in outcomes related to balance.

All studies reported outcomes at baseline and at intervention end (endpoint values), except for Kluding 2013, which presented outcomes as the change from baseline values. However, we were able to extract all outcomes from Kluding 2013 because they were presented in an reference associated with the study (Dunning 2015). Only one study had repeated observations of participants, with an assessment of outcomes in the middle part of the intervention period (Bethoux 2014), and only one study assessed follow-up 12 and 24 weeks post-treatment (Sheffler 2013a).

All of these outcomes except those related to participation/quality of life were assessed either while the participants were using MN or while they were not using MN. Two studies performed final assessments while participants were using MN: one study investigated the training effect of the intervention, so the baseline assessment was performed while the participants used $\mathrm{MN}$ (Bethoux 2014), while the other study investigated the total effect of intervention, so the baseline assessment was performed while the participants were not using MN (Kottink 2007). One study assessed the training effect of $\mathrm{MN}$, thus all assessments were performed without the use of MN (Sheffler 2013a). One study evaluated both the training and therapeutic effect, so assessment of each outcome was conducted while participants were using MN and while they were not using MN (Kluding 2013).
All studies reported withdrawal or dropouts for several reasons during the intervention period (Table 3). Only two studies reported data for serious adverse events related to the intervention (Bethoux 2014; Kluding 2013). Although Sheffler 2013a included data for serious adverse events in its trial registry, it was not clear which data were related to the intervention, therefore we did not extract these data. Three studies presented data for falls (Bethoux 2014; Kluding 2013; Sheffler 2013a). Kottink 2007 did not mention any adverse event data in its reports.

\section{Excluded studies}

We excluded 68 studies (see Characteristics of excluded studies and Figure 1 for further information).

\section{Studies awaiting assessment}

Four studies are still awaiting assessment. We were unable to contact the principal investigator of one study because our email was undelivered (Wright 2004). We contacted the principal investigators of ISRCTN91639560 and NCT03574623 to learn if the electrical stimulation was used as an orthosis in the home or community context, but we have not received a response to date. We also contacted the principal investigator of UMIN000018648, who stated that the electrical stimulation protocol was applied at home. To date, we have received no response clarifying whether the study has already been published. See Characteristics of studies awaiting classification.

\section{Ongoing studies}

We identified one ongoing study that appeared to be eligible for inclusion (Ghedira 2014). See Characteristics of ongoing studies.

\section{Risk of bias in included studies}

'Risk of bias' assessments are presented for individual studies in Characteristics of included studies. See Figure 2 and Figure 3 for summaries of the results.

Figure 2. Risk of bias graph: review authors' judgements about each risk of bias item presented as percentages across all included studies.

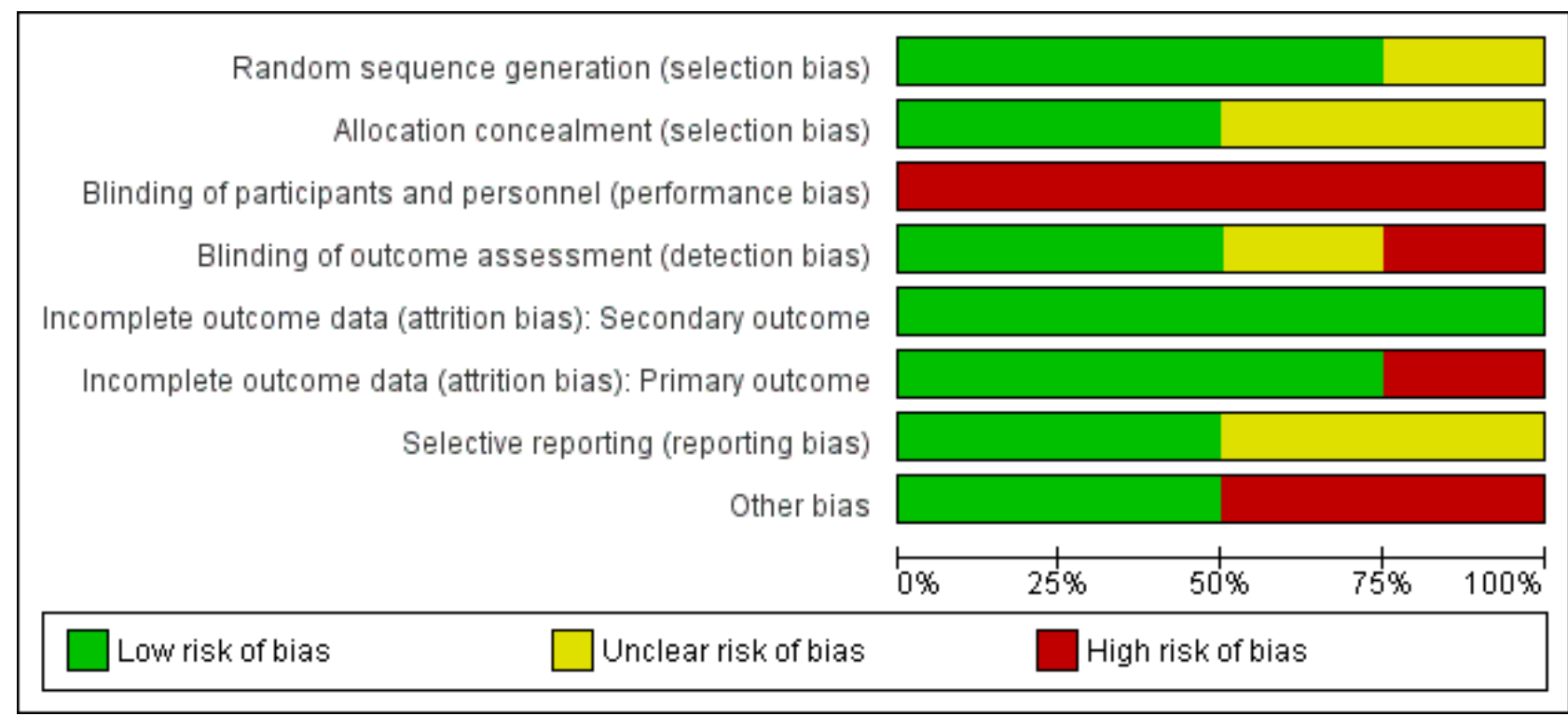


Figure 3. Risk of bias summary: review authors' judgements about each risk of bias item for each included study.

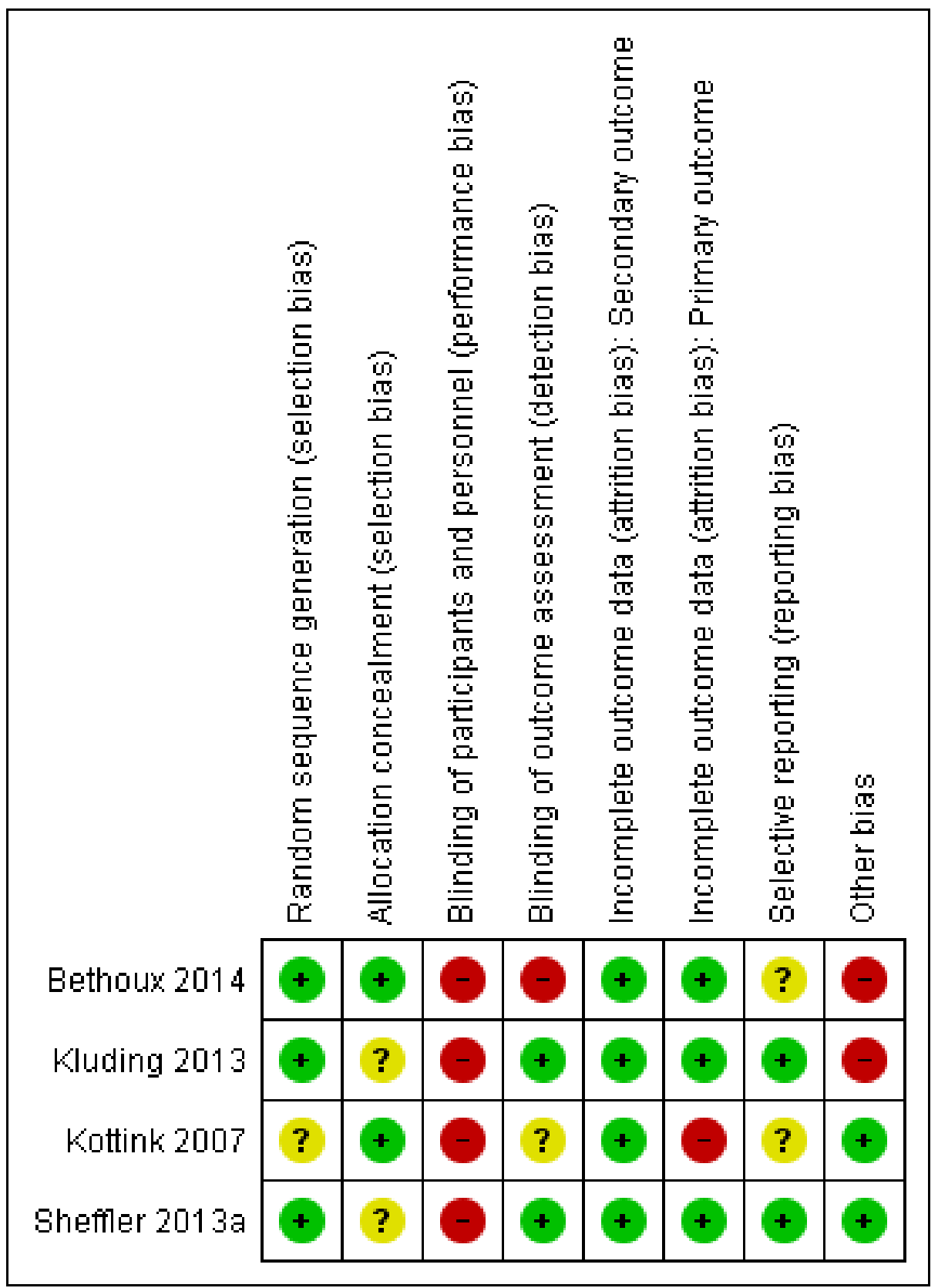

\section{Allocation}

\section{Sequence generation}

Generation of randomization sequence was conducted correctly in three studies (Bethoux 2014; Kluding 2013; Sheffler 2013a), which we deemed to be at low risk of bias. One study did not clearly report if the method used for selecting the blocks described a random component in the sequence generation process, therefore we classified it as at unclear risk of bias (Kottink 2007).

\section{Allocation concealment}

We judged two trials to be at low risk of bias for allocation concealment (Bethoux 2014; Kottink 2007). We considered the other two included studies to be at unclear risk of bias: Kluding 2013 because the method of concealment was not described in sufficient detail to permit a definitive judgement, and Sheffler 2013a because the investigators did not report whether the envelopes used were sealed or not.

\section{Blinding}

\section{Blinding of participants and personnel}

None of the studies utilized blinded participants or personnel because of the nature of the intervention. We judged all studies as having a high risk of detection bias. 


\section{Blinding of outcome assessment}

We assessed two studies where the outcome assessors were blinded to treatment allocation as at low risk of detection bias (Kluding 2013; Sheffler 2013a). One study had a high risk of detection bias because outcome assessments were unblinded (Bethoux 2014), whereas another study provided insufficient information to permit an assessment of level of bias and was therefore classified as at unclear risk of bias (Kottink 2007).

\section{Incomplete outcome data}

All studies reported withdrawals or dropouts, but we classified them as having a low risk of bias considering that ITT analyses were performed. Only Kottink 2007 did not perform ITT analysis for the primary outcome, hence we classified it as having a high risk of detection bias.

\section{Selective reporting}

We classified two studies as having a low risk of selective reporting because they had protocols available, and all of the prespecified outcomes were reported in the prespecified way (Kluding 2013; Sheffler 2013a). We considered two studies as having an unclear risk of selective reporting: Bethoux 2014 included a secondary variable in the study that was not prespecified in the protocol, and Kottink 2007 provided insufficient information to permit a judgement.

\section{Other potential sources of bias}

We assessed two studies that were sponsored by manufacturers of MN as having a high risk of bias (Bethoux 2014; Kluding 2013). No other bias was detected in Kottink 2007 and Sheffler 2013a.

\section{Effects of interventions}

See: Summary of findings for the main comparison Motor neuroprosthesis compared to another assistive technology device for promoting recovery of function after stroke

See Summary of findings for the main comparison.

We included all four studies in the quantitative analysis (Bethoux 2014; Kluding 2013; Kottink 2007; Sheffler 2013a). All studies compared MN versus another assistive technology device. The outcomes used in these studies were: activities involving limbs (Bethoux 2014; Kluding 2013; Kottink 2007; Sheffler 2013a); participation scales of HRQoL (Bethoux 2014; Kluding 2013; Kottink 2007; Sheffler 2013a); exercise capacity (Bethoux 2014; Kluding 2013); balance (Bethoux 2014; Kluding 2013); number of dropouts (Bethoux 2014; Kluding 2013; Kottink 2007; Sheffler 2013a); serious adverse events related to intervention (Bethoux 2014; Kluding 2013); and falls (Bethoux 2014; Kluding 2013; Sheffler 2013a).

We contacted the principal investigator of Kottink 2007 to request data for outcomes of the 6MWT and walking speed assessed with ITT analysis with and without devices, which were presented in Kottink 2007 and Kottink 2008 only as figures. However, we could not obtain these data, so we excluded the 6MWT results of this study from the quantitative analysis and extracted the walking numerical speed data analyzed without ITT presented in Kottink 2012.

We considered for meta-analysis only Kluding 2013 assessments performed with the participants using a device (MN or another assistive device). The study of Bethoux 2014 had two publications that performed an assessment at different time intervals during the intervention period (repeated observations of the participants). In order to gain a better understanding of the effect of $\mathrm{MN}$ on different time periods, we decided to include data from both Bethoux 2014 publications in the meta-analysis.

Kluding 2013 used two measures to assess walking speed: comfortable and fast walking speed. As all studies assessed comfortable walking speed, and no study assessed fast walking speed, we decided to include only the Kluding 2013 data for comfortable walking speed in the meta-analysis.

\section{Comparison: motor neuroprosthesis versus another assistive technology device}

\section{Independence in $A D L$}

None of the four included studies reported outcomes related to independence in ADL.

\section{Activities involving limbs}

\section{Walking speed until six months of device use}

Two studies (605 participants) measured walking speed until six months of device use. One study performed a final assessment with participants using $\mathrm{MN}$; the other study did not perform a final assessment with participants using MN. We found lowcertainty evidence that the control intervention (another assistive technology device) had a greater effect than MN on walking speed in six months of device use: the mean difference (MD) (randomeffects model) was $-0.05 \mathrm{~m} / \mathrm{s}$ (95\% confidence interval $(\mathrm{Cl})-0.10$ to $-0.00 ; P=0.03 ; 1^{2}=0 \%$; Analysis 1.1 ). But considering that the minimal important difference for comfortable walking speed in chronic stroke participant is $0.2 \mathrm{~m} / \mathrm{s}$, this effect was not enough to be clinically meaningful.

We conducted sensitivity analysis by excluding Bethoux 2014 since this study presented a high risk of bias in the blinding of outcome assessment. The sensitivity analysis showed that there is low certainty that the effect of the control intervention on improving walking speed is no longer present (MD $-0.07 \mathrm{~m} / \mathrm{s}, 95 \% \mathrm{Cl}-0.16$ to $0.02 ; \mathrm{P}=0.13 ; \mathrm{I}^{2}=0 \% ; 110$ participants; Table 4).

\section{Walking speed between six and 12 months of device use}

Three studies (713 participants) measured the walking speed of participants between six and 12 months of device use. All three studies performed final assessments while the participants used $\mathrm{MN}$. There is low-certainty evidence that $\mathrm{MN}$ is no more beneficial than another assistive device on walking speed between six and 12 months of device use (MD $0.00 \mathrm{~m} / \mathrm{s}, 95 \% \mathrm{Cl}-0.05$ to $0.05 ; \mathrm{P}=0.93$; $\mathrm{I}^{2}=17 \%$; Analysis 1.2).

We conducted a sensitivity analysis excluding the study that was at high risk of bias for blinding of outcome assessment, which highlighted that we are very uncertain whether MN is more beneficial than another assistive device on walking speed between six and 12 months of device use (Table 4). Kottink 2007 had a high risk of bias for incomplete outcome data relating to this outcome, but as we did not consider this 'Risk of bias' domain on sensitivity analysis, we decided to maintain these data. However, caution should be used in interpreting these data as their results were visually different from data for other studies. 


\section{Walking speed: subgroup analysis for type of $\mathrm{MN}$}

We analyzed subgroups considering the type of MN used (823 participants). We compared studies in which the MN used consisted of a superficial device with those in which MN consisted of an implantable device. For this subgroup analysis, we considered the walking speed assessment performed in Bethoux 2014 at 12 months. The test for subgroup differences (between surface $M N$ and implantable $M N)$ revealed no significant difference $\left(P=0.09 ; 1^{2}=\right.$ 65.1\%; Analysis 1.3).

\section{TUG at the end of the intervention phase}

Two studies (692 participants) assessed TUG. In both studies, the final assessments of participants were performed while they were using MN. As Bethoux 2014 presented TUG assessment only for six months, these data were included for TUG analysis. There is moderate-certainty evidence that $\mathrm{MN}$ is no more beneficial than another assistive device on TUG (MD $0.51 \mathrm{~s}, 95 \% \mathrm{Cl}-4.41$ to 5.43; P $=0.84 ; 1^{2}=0 \%$; Analysis 1.4 ).

The sensitivity analysis performed by excluding Bethoux 2014, which was at high risk of bias for blinding of outcome assessment, highlighted that more information is required to be certain as to whether MN is no more beneficial than another assistive device on TUG (Table 4).

\section{mEFAP at the end of the intervention phase}

Two studies (605 participants) assessed mEFAP. One study performed a final assessment of participants while using MN, whereas the other study did not perform a final assessment of the participants while using MN. As Bethoux 2014 presented mEFAP assessment only for six months, these data were included for mEFAP analysis. There is low-certainty evidence that MN is no more beneficial than another assistive device on mEFAP (MD $14.77 \mathrm{~s}, 95 \%$ $\mathrm{Cl}-12.52$ to $42.06 ; \mathrm{P}=0.29 ; \mathrm{I}^{2}=0 \%$; Analysis 1.5 ).

The sensitivity analysis performed by excluding Bethoux 2014 data highlighted that more information is required to be certain as to whether MN is no more beneficial than another assistive device on mEFAP (Table 4).

\section{Participation scales of HRQOL}

All studies assessed at least one participation scale of HRQoL. There was heterogeneity between the selection of scales of HRQoL as well as their presentation (some scales presented the total value of a full version, while others presented the value of some domains separately). In light of this, we decided to include only scales or scale components that represented the whole domain of a scale and to combine data from these different scales using standardised mean difference (SMD) as stated in our protocol. Bethoux 2014 presented two participation scales of HRQoL at six months' assessment; we decided to use the SSQoL for analysis, as the total value was available. We did not include measures that represented only some domains of a scale of HRQoL. We included data from three studies (632 participants) that assessed HRQoL with a participation scale. There is very low-certainty evidence that $\mathrm{MN}$ is no more beneficial than another assistive device on participation scale of $\mathrm{HRQ}$ oL. The random-effects pooled estimate for all trials was SMD $0.26\left(95 \% \mathrm{Cl}-0.22\right.$ to $0.74 ; \mathrm{P}=0.28 ; \mathrm{I}^{2}=77 \%$; Analysis 1.6).
The sensitivity analysis performed by excluding Bethoux 2014 data highlighted that we are very uncertain as to whether $\mathrm{MN}$ is any more beneficial than another assistive device on participation scale of $\mathrm{HRQ}$ oL, although the magnitude of the effect changed from a small effect (Analysis 1.6) to a moderate effect based on Cohen's rules of thumb (Table 4).

\section{Exercise capacity}

Two studies (692 participants) assessed exercise capacity using the 6MWT. Both studies performed final assessments on participants using MN. As Bethoux 2014 presented 6MWT assessment only for six months, these data were included for 6MWT analysis. There is low-certainty evidence that MN is no more beneficial than another assistive device on exercise capacity (MD $-9.03 \mathrm{~m}, 95 \% \mathrm{Cl}-26.87$ to 8.81; $P=0.32 ; 1^{2}=0 \%$; Analysis 1.7 .

The sensitivity analysis performed by excluding Bethoux 2014, which was at high risk of bias for blinding of outcome assessment, highlighted that more information is required to be certain as to whether MN is no more beneficial than another assistive device on exercise capacity (Table 4).

\section{Balance}

Two studies (692 participants) assessed balance using the BBS. Both studies performed final assessments on participants using $\mathrm{MN}$. There is moderate-certainty evidence that $\mathrm{MN}$ is no more beneficial than another assistive device on balance (MD - 0.34, 95\% $\mathrm{Cl}-1.96$ to $1.28 ; \mathrm{P}=0.68 ; \mathrm{I}^{2}=0 \%$; Analysis 1.8 ).

The sensitivity analysis excluding Bethoux 2014 data highlighted that we are very uncertain as to whether $\mathrm{MN}$ is more beneficial than another assistive device on balance (Table 4).

\section{Number of dropouts}

All studies (829 participants) reported dropouts during the intervention period; the reasons for dropouts are described in detail for each trial in Table 3. For this outcome, we considered the number of dropouts for Bethoux 2014 at 12 months. There is lowcertainty evidence that the risk of participants dropping out of the study was increased by $51 \%$ with MN when compared with control. The risk ratio (RR) (random-effects model) for dropouts was 1.48 $\left(95 \% \mathrm{Cl} 1.11\right.$ to $1.97 ; \mathrm{P}=0.007 ; \mathrm{I}^{2}=0 \%$; Analysis 1.9$)$. The highest dropout rate occurred in Bethoux 2014 (12 months of intervention): $26 \%$ in the MN group (62 dropouts out of 242 participants) and $19 \%$ in the control group ( 49 dropouts out of 253 participants). The lowest dropout rate occurred in Kottink 2007: 7\% in the MN group ( 1 dropout out of 14 participants) and $8 \%$ in the control group (1 dropout out of 13 participants).

\section{Adverse events}

Only one study reported deaths during the intervention period (Bethoux 2014). The death rate was less than $1 \%$ for both groups in the six-month intervention report of Bethoux 2014. In the MN group, deaths were due to a nervous system disorder or renal and urinary disorders ( 2 deaths of 242 participants), whereas deaths in the control group were due to nervous system disorders ( 2 deaths of 253 participants). In the 12-month intervention report of the Bethoux 2014 study (Bethoux 2015), the death rate was maintained in the MN group (less than 1\%; 2 deaths of 242 participants), but was increased by $1 \%$ in the control group (3 deaths of 253 participants). 


\section{Serious adverse events related to intervention}

Two studies (692 participants) provided data on serious adverse events related to the intervention during the treatment period. Bethoux 2014 considered serious adverse events related to device use as serious falls; as this study presented this outcome in both six- and 12-month reports, we decided to include the longer assessment (12 months). Overall, there is low-certainty evidence that the use of $\mathrm{MN}$ in people after stroke does not have an effect on risk of adverse events during the treatment period when compared to other assistive devices: RR (random-effects model) of 0.35 (95\% $\mathrm{Cl} 0.04$ to $3.33 ; \mathrm{P}=0.36 ; \mathrm{I}^{2}=0 \%$; Analysis 1.10$)$.

\section{Falls}

Three studies (802 participants) provided data on falls. There is moderate-certainty evidence that the use of $\mathrm{MN}$ in people after stroke does not have an effect on risk of falls during the whole treatment period when compared to other assistive devices: RR (random-effects model) of $1.20(95 \% \mathrm{Cl} 0.92$ to $1.55 ; \mathrm{P}=0.08 ; \mathrm{l} 2=$ 33\%; Analysis 1.11).

\section{DISCUSSION}

\section{Summary of main results}

The aim of this review was to assess the effects of MN for improving independence in ADL, activities involving limbs, participation scales of HRQoL, exercise capacity, balance, and adverse events in people after stroke. We included four studies ( 9 articles) involving a total of 831 participants that compared $\mathrm{MN}$ with another assistive technology device. All studies addressed MN application directed to the lower limbs, specifically in the peroneal nerve, to correct foot drop during walking activities in the home or community context, such as an orthosis. In all studies, another assistive technology device (control intervention) used was ankle-foot orthosis (AFO). No studies compared MN with no treatment or with MN without electrical stimulus. Overall, the certainty of the evidence for outcomes ranged from moderate to very low. The main results are presented in the Summary of findings for the main comparison.

No studies reported outcomes related to independence in ADL, so we could not assess the evidence of the effects of MN on ADL. Although we found low-certainty evidence that AFO had an effect on improving walking speed until six months of device use (MD $-0.05 \mathrm{~m} / \mathrm{s}, 95 \% \mathrm{Cl}-0.10$ to -0.00 ), this effect did not appear to be clinically relevant, given that the minimally significant difference for comfortable walking speed in chronic stroke participants is 0.2 $\mathrm{m} / \mathrm{s}$ (Hiengkaew 2012). Furthermore, when we excluded one study at high risk of bias related to unblinded outcome assessment from the meta-analysis, this effect was absent, that is both strategies (MN and AFO) proved to have similar effects on walking speed until six months of device use. We found low-certainty evidence that there is no beneficial effect of $\mathrm{MN}$, when compared to another assistive device, for walking speed between six and 12 months of device use. We also observed no difference in effect on walking speed between the surface and implantable MN. We have very little confidence in our estimate of the effect of implantable $M N$ because only one small study with a high risk of bias for incomplete outcome data and unclear risk for random sequence generation, blinding of outcome assessment, and selective reporting investigated its effect. For this reason, the exact effect of implantable $\mathrm{MN}$ is likely to be substantially different from the estimate of its effect.
We found moderate-certainty evidence that MN has no effect on balance measured with BBS and activities involving limbs such as TUG. We found low-certainty evidence that MN has no effect on exercise capacity measured with 6MWT and activities involving limbs such as mEFAP. Although there were some limited moderateor low-certainty evidence, this apparent lack of effect ( $M N$ is not any different to AFO) should be interpreted with caution due to the high risk of bias (outcome assessors were unblinded) in the largest study and the broad confidence intervals these outcomes presented. Regarding the secondary outcome measure participation scale of $\mathrm{HRQ}$ L, we found very low-certainty evidence that MN does not differ in effect compared to AFO. However, due to the quality of evidence, any potential benefits of the interventions remain uncertain.

We found low-certainty evidence that the use of MN in people after stroke increases the risk of participants dropping out of the study. However, when considering safety, we found that the number of falls (moderate-certainty evidence) and the number of serious adverse events (low-certainty evidence) were not increased related to the use of MN. We considered serious adverse events related to device use as serious falls. Limited data contributed to the results for this outcome, and due to the wide confidence intervals, further information is needed.

\section{Overall completeness and applicability of evidence}

Our search results identified a considerable number of studies that applied MN devices in a clinical context for ADL training or even as a home-based program to increase the dose therapy usually done with cyclic stimulation. As this was not the focus of this review, we excluded these studies. We found only four studies (nine articles) that focused on the effects of MN for improving activities and participation in people after stroke, considering its use as an environmental facilitator to enhance the performance of functional abilities in the home or community context. While the number of such studies was small, the number of participants was not (831 participants). We also found trials that used the brain-machine interface (BMI) to control the signals of electrical stimulation devices; however, no study met the inclusion criteria of this review, especially with regard to study design.

The results of this review indicate that little is known about the effect of MN and that further information is needed. There is currently insufficient high-certainty evidence to make conclusions about the benefits or harms of MN. Overall, there is no substantial evidence that $\mathrm{MN}$ has an effect on improving activities involving limbs, participation scales of HRQoL, exercise capacity, balance, and adverse events. The only possible effect found was that the use of $\mathrm{MN}$ in people after stroke increased the risk of participants dropping out the study for several reasons, which included participant request to discontinue the intervention, lost to followup, medical reasons, non-compliance with protocol, and others as mentioned in Table 3.

Considering that we only included studies with MN applied on the lower limb, these results cannot be generalized to include improvement of activities related to the upper limb. Even considering MN directed to the lower limb, the following factors produce uncertainty.

- The majority of interventions were focused on single-channel surface MN for stimulation of the peroneal nerve. 
- All interventions consisted of MN applied on the peroneal nerve to facilitate walking activities.

- The activities performed and their duration throughout the conditioning protocols varied between studies. These conditioning protocols prepared participants for $\mathrm{MN}$ use for a long period during the day, and all studies included them in the first few weeks of the intervention period.

As all the included studies used non-biological signals such as force/pressure and inertial sensors as an input to trigger the electrical stimulus, the results of this review cannot be generalized for $\mathrm{MN}$ triggered with electromyography or electroencephalography signals. Since we considered the use of $\mathrm{MN}$ as an orthosis, the only comparison we found was MN versus another assistive technology device. In this way, this review provides incipient data that can help in decision making on the use of MN or AFO, not considering other assistive devices or MN versus no device.

Since none of the studies included participants within three months of stroke, we did not assess if the effects of MN can be generalized for individuals in the early stages of stroke. In addition, the mean walking speed at baseline assessment varied widely between studies that assessed superficial and implantable MN devices.

The most common outcome was walking speed, which was part of activities involving limbs, but no study assessed other important outcomes for people with stroke, such as independence in ADL. Consequently, there is a need to monitor this outcome in future updates to determine the effect of $M N$ on independence in $A D L$.

Additionally, MN using an environmental facilitator to enhance the performance of functional abilities in the home or community context could possibly create additional costs of rehabilitation after stroke due to the nature of the device and because the structure requires experts to fit, adapt, and train users to use the device; researchers did not quantify the costs of its application. In this context, the results of this review seem to be quite generalizable for industrialized countries that have services and facilities available for the application and adaptation of MN to users.

\section{Quality of the evidence}

According to the GRADE criteria, the certainty of evidence ranged from moderate to very low due to the small number of studies included in the review, which led to wide confidence intervals for seven out of the nine included outcomes, and the presence of some judgements of high risk and unclear risk of detection bias in the included studies (see Summary of findings for the main comparison). Three out of four trials showed a high risk of bias for one or two of these 'Risk of bias' domains: blinding of outcome assessment, incomplete outcome data for primary outcome, or other potential sources of bias. The outcome assessors in the largest study were not blinded. The two most significant studies, which represented $83 \%$ of included participants, were multicenter and sponsored by manufacturers of $\mathrm{MN}$; because of this, we classified them as being at high risk of other potential sources of bias. However, these studies adopted some precautions that may have minimized the presence of other biases, such as clearly described adverse events related and not related to $\mathrm{MN}$ and AFO groups on trial registries, sufficient methodological detail presented with prior protocol publication, or even inclusion of an independent Clinical Events Committee to adjudicate serious adverse events and their connection to the device. All studies had a high risk of bias for blinding participants or personnel because of the nature of the intervention. Poor reporting and lack of clarification from the authors led us to assess studies as being at unclear risk of bias for important criteria such as random sequence generation, allocation concealment, blinding of outcome assessment, and selective reporting. Nevertheless, most of the results were consistent (low heterogeneity).

\section{Potential biases in the review process}

Given our extensive searching process, we are confident that our search strategy was comprehensive and detailed; this strategy included searching in databases, electronic registries, websites, and a careful search of grey literature. We thus expect that we have identified all relevant studies; however, there is a small possibility that we failed to identify additional (published or unpublished) papers. Two review authors independently assessed the studies and obtained and extracted data, with a third or fourth review author available to resolve disagreements as needed, thereby minimizing bias; several subjective judgements were required during the review process.

We decided to downgrade our assessment of blinding of participants and personnel, even while knowing that such blinding does not seem feasible for the type of intervention. Another limitation of this review is that some of the studies had methodological shortcomings, such as random sequence generation, allocation concealment, blinding of outcome assessment, incomplete outcome data for the primary outcome, and selective reporting. According to the Cochrane Handbook for Systematic Reviews of Interventions, these biases can lead to overestimation of the intervention effect (Higgins 2011b).

\section{Agreements and disagreements with other studies or reviews}

We found only one systematic review with meta-analysis of randomized controlled trials on $\mathrm{MN}$ for improving activities and participation in people after the stroke that considered $\mathrm{MN}$ use as an environmental facilitator to enhance the performance of functional abilities (Prenton 2016). Although that review focused on $\mathrm{MN}$ directed to peroneal nerve stimulation as well, it differed from the current review by including studies that used MN in the ward environment, which did not represent a real-life context. The authors referred to the MN device with the use of FES nomenclature. As the comparison included in Prenton 2016 was $M N$ versus AFO on the date of the final assessment of 10-meter walking speed and 6MWT, its results were similar to the results of the current review, which indicated that AFO had positive orthotic effects on walking that are equivalent to FES for foot drop on stroke participants. Similarly, researchers also found little difference in favor of AFO for evaluations performed between 12 and 13 weeks of device use, but they did not perform sensitivity analysis. Prenton 2016 additionally performed a meta-analysis for the mobility domain of the Stroke Impact Scale and found no difference between the two interventions, but did not assess the dropout rate between studies.

As far as we know, no systematic review has evaluated the number of dropouts related of MN use with meta-analysis. There are only separated reports that assess compliance and preference for MN. Among the studies included in this review, only Kluding 2013 
evaluated satisfaction related to the use of the devices. The authors of this study used a satisfaction survey that evaluates 12 items with a total range of scores from 0 to 24 , with a higher number indicating greater satisfaction with the device. Although there was a higher dropout rate in the MN group (25 of 99 participants) than in the AFO group (10 of 98 participants), the satisfaction was higher in the MN group than in the AFO group. Everaert 2013 recorded the users' preference and asked participants to indicate the reasons for their preference at the end of each arm of a crossover study. The majority of participants preferred the MN, the reasons most frequently mentioned being function, confidence, comfort, convenience, easy donning and doffing, and safety.

\section{AUTHORS' CONCLUSIONS}

\section{Implications for practice}

Overall, motor neuroprosthesis (MN) (use of electrical stimulation as an environmental facilitator in the home or community context) does not appear to be more beneficial than other assistive devices for improving activities involving limbs measured by Timed Up and Go, balance (moderate-certainty evidence), activities involving limbs measured by walking speed and modified Emory Functional Ambulation Profile, exercise capacity (low-certainty evidence), and participation scale of health-related quality of life (very lowcertainty evidence). As such, $\mathrm{MN}$ is not any different to anklefoot orthosis for the above mentioned outcomes. We could not estimate the effect of MN on independence in activities of daily living because no study assessed this outcome. Although there was moderate certainty that MN did not increase the number of falls and low certainty that MN did not increase serious adverse events related to the intervention during the intervention period, the number of dropouts related to MN use was higher than with the control. Considering the low certainty of the evidence for this outcome (dropouts), our confidence in this effect estimate is limited and needs to be confirmed in future clinical trials.

Because of the specificity found in the included studies that assessed the effects of $\mathrm{MN}$ directed to the lower limb in participants more than three months' poststroke, it is not possible to generalize the aforementioned effects to participants less than three months' poststroke and interventions such as upper limb MN or MN triggered by electromyography or electroencephalography signals. Concerning implantable $\mathrm{MN}$, the apparent lack of effect of implantable MN should be interpreted with caution due to the very low certainty of the evidence, which means that the effect of lower limb implantable MN on walking speed could be very different from the estimated effect. Further investigation is needed in this regard.

\section{Implications for research}

Further research should improve the certainty of the evidence (GRADE) regarding the effect of $\mathrm{MN}$ for improving activities and participation in people after stroke. New well-designed and properly reported randomized controlled clinical trials should be conducted using a larger sample in order to provide high-quality evidence, preferably with blinded outcome assessment.

In order to understand the effects of the whole MN category on activities and participation in people after stroke, it is necessary to design randomized controlled trials with $\mathrm{MN}$ directed to the upper limb as well as with implantable devices and devices that use biological signals to trigger the stimulation. These studies should focus on MN use as an environmental facilitator for enhancing the performance of functional abilities in the home or community context, especially involving outcomes related to independence in activities of daily living. As we found a higher dropout rate with $\mathrm{MN}$ use, it is important to thoroughly consider the motivations of each participant in relation to compliance or satisfaction in order to understand the cause of higher MN dropouts, as well as to guide future research on the development of these devices.

Considering that there is a variable and prolonged process ranging from the fitting of the MN to the participant's limb to its use as an assistive device for enhancing the performance of functional abilities in the home or community context, it is necessary to assess the outcomes related to activity and participation during all-day use of MN period separately from the conditioning protocols period. In this way it will be possible to precisely assess the use of $\mathrm{MN}$ as an orthosis without the contamination of previous conditioning protocols data, as was observed in all studies included in this review.

\section{ACKNOWLEDGEMENTS}

We thank Hazel Fraser, Cochrane Stroke Group Managing Editor; Joshua Cheyne, Cochrane Stroke Group Information Specialist; Peter Langhorne, Cochrane Stroke Group Co-ordinating Editor; Jan Mehrholz and Alex Pollock, Cochrane Stroke Group Associate Editors; Aryelly Rodriguez, Cochrane Stroke Group Statistical Editor; Bernhard Elsner, Cochrane author, Department of Public Health, Technical University Dresden; and Dee Shneiderman and Sunita Gudwani, consumer reviewers, for their valuable advice on writing the protocol and the review. 


\section{R E F E R E N C E S}

\section{References to studies included in this review}

\section{Bethoux 2014 \{published data only\}}

Bethoux F, Rogers HL, Nolan KJ, Abrams GM, Annaswamy T, Brandstater $\mathrm{M}$, et al. Long-term follow-up to a randomized controlled trial comparing peroneal nerve functional electrical stimulation to an ankle foot orthosis for patients with chronic stroke. Neurorehabilitation and Neural Repair 2015;29(10):911-22.

* Bethoux F, Rogers HL, Nolan KJ, Abrams GM, Annaswamy TM, Brandstater $\mathrm{M}$, et al. The effects of peroneal nerve functional electrical stimulation versus ankle-foot orthosis in patients with chronic stroke: a randomized controlled trial. Neurorehabilitation and Neural Repair 2014;28(7):688-97.

\section{Kluding 2013 \{published data only\}}

Kluding PM, Dunning K, O'Dell MW, Wu SS, Ginosian J, Feld J, et al. Foot drop stimulation versus ankle foot orthosis after stroke: 30-week outcomes. Stroke 2013;44(6):1660-9.

\section{Kottink 2007 \{published data only\}}

* Kottink Al, Hermens HJ, Nene AV, Tenniglo MJ, van der Aa HE, Buschman HP, et al. A randomized controlled trial of an implantable 2-channel peroneal nerve stimulator on walking speed and activity in poststroke hemiplegia. Archives of Physical Medicine and Rehabilitation 2007;88(8):971-8.

Kottink Al, ljzerman MJ, Groothuis-Oudshoorn CG, Hermens HJ. Measuring quality of life in stroke subjects receiving an implanted neural prosthesis for drop foot. Artificial Organs 2010;34(5):366-76.

Kottink Al, Tenniglo MJ, de Vries WH, Hermens HJ, Buurke JH. Effects of an implantable two-channel peroneal nerve stimulator versus conventional walking device on spatiotemporal parameters and kinematics of hemiparetic gait. Journal of Rehabilitation Medicine 2012;44(1):51-7.

Kottink AIR, Hermens HJ, Nene AV, Tenniglo MJ, GroothuisOudshoorn CG, ljzerman MJ. Therapeutic effect of an implantable peroneal nerve stimulator in subjects with chronic stroke and footdrop: a randomized controlled trial. Physical Therapy 2008;88(4):437-48.

\section{Sheffler 2013a \{published data only\}}

Sheffler LR, Taylor PN, Bailey SN, Gunzler DD, Buurke JH, IJzerman MJ, et al. Surface peroneal nerve stimulation in lower limb hemiparesis. American Journal of Physical Medicine and Rehabilitation 2015;94(5):341-57.

* Sheffler LR, Taylor PN, Gunzler DD, Buurke JH, ljzerman MJ, Chae J. Randomized controlled trial of surface peroneal nerve stimulation for motor relearning in lower limb hemiparesis. Archives of Physical Medicine and Rehabilitation 2013;94(6):1007-14.

\section{References to studies excluded from this review}

Alon 2002 \{published data only\}

Alon G, McBride K, Ring H. Improving selected hand functions using a noninvasive neuroprosthesis in persons with chronic stroke. Journal of Stroke and Cerebrovascular Diseases 2002;11(2):99-106.

\section{Alon 2003a \{published data only\}}

Alon G, Ring H. Gait and hand function enhancement following training with a multi-segment hybrid-orthosis stimulation system in stroke patients. Journal of Stroke and Cerebrovascular Diseases 2003;12(5):209-16.

\section{Alon 2007 \{published data only\}}

Alon G, Levitt AF, McCarthy PA. Functional electrical stimulation enhancement of upper extremity functional recovery during stroke rehabilitation: a pilot study. Neurorehabilitation and Neural Repair 2007;21(3):207-15.

\section{Alon 2008 \{published data only\}}

Alon G, Levitt AF, McCarthy PA. Functional electrical stimulation (FES) may modify the poor prognosis of stroke survivors with severe motor loss of the upper extremity: a preliminary study. American Journal of Physical Medicine and Rehabilitation 2008;87(8):627-36.

Baker 2004 \{published data only\}

Baker LL, Palmer E, Waters RL, Chun SN. Rehabilitation of the arm and hand following stroke-a clinical trial with BIONs. The 26th Annual International Conference of the IEEE Engineering in Medicine and Biology Society; 2004 Sep 1-5; San Francisco (CA). https://ieeexplore.ieee.org/document/1404168, 2004:4186-8.

\section{Barrett 2010 \{published data only\}}

Barrett C, Taylor P. The effects of the Odstock drop foot stimulator on perceived quality of life for people with stroke and multiple sclerosis. Neuromodulation 2010;13(1):58-64.

\section{Berner 2004 \{published data only\}}

Berner YN, Kimchi OL, Spokoiny V, Finkeltov B. The effect of electric stimulation treatment on the functional rehabilitation of acute geriatric patients with stroke-a preliminary study. Archives of Gerontology and Geriatrics 2004;39(2):125-32.

\section{Bundy 2017 \{published data only\}}

Bundy DT, Souders L, Baranyai K, Leonard L, Schalk G, Coker R, et al. Contralesional brain-computer interface control of a powered exoskeleton for motor recovery in chronic stroke survivors. Stroke 2017;48(7):1908-15.

\section{Burridge 1997a \{published data only\}}

Burridge JH, Taylor PN, Hagan SA, Wood DE, Swain ID. The effects of common peroneal stimulation on the effort and speed of walking: a randomized controlled trial with chronic hemiplegic patients. Clinical Rehabilitation 1997;11(3):201-10. 


\section{Burridge 1997b \{published data only\}}

Burridge J, Taylor P, Hagan S, Wood D, Swain I. The effect of common peroneal nerve stimulation on quadriceps spasticity in hemiplegia. Physiotherapy 1997;83(2):82-9.

\section{Burridge 1997c \{published data only\}}

Burridge J, Taylor P, Hagan S, Swain I. Experience of clinical use of the Odstock dropped foot stimulator. Artificial Organs 1997;21(3):254-60.

\section{Burridge 2007a \{published data only\}}

Burridge J, Haugland M, Larsen B, Pickering RM, Svaneborg N, Iversen HK, et al. Phase II trial to evaluate the ActiGait implanted drop-foot stimulator in established hemiplegia. Journal of Rehabilitation Medicine 2007;39(3):212-8.

\section{Burridge 2007b \{published data only\}}

Burridge JH, Elessi K, Pickering RM, Taylor PN. Walking on an uneven surface: the effect of common peroneal stimulation on gait parameters and relationship between perceived and measured benefits in a sample of participants with a drop-foot. Neuromodulation 2007;10(1):59-67.

\section{Burridge 2011 \{published data only\}}

Burridge JH, Turk R, Merrill D, Dibb B, Hughes AM, Sparrow O, et al. A personalized sensor-controlled microstimulator system for arm rehabilitation poststroke. Part 2: Objective outcomes and patients' perspectives. Neuromodulation 2011;14(1):80-8.

\section{Chae 2009 \{published data only\}}

Chae J, Harley MY, Hisel TZ, Corrigan CM, Demchak JA, Wong YT, et al. Intramuscular electrical stimulation for upper limb recovery in chronic hemiparesis: an exploratory randomized clinical trial. Neurorehabilitation and Neural Repair 2009;23(6):569-78.

\section{Chan 2009 \{published data only\}}

Chan MK, Kai-yu TR, Yiu-kwan CK. Bilateral upper limb training with functional electric stimulation in patients with chronic stroke. Neurorehabilitation and Neural Repair 2009;23(4):357-65.

\section{ChicTR-IOR-17013339 \{published data only\}}

ChiCTR-IOR-17013339. A randomized controlled trial for effects and mechanism of intelligent functional electrical stimulation on ambulation of stroke patients. www.chictr.org.cn/ showprojen.aspx?proj=22207 (first received 10 November 2017).

\section{Daly 2011 \{published data only\}}

Daly JJ, Zimbelman J, Roenigk KL, McCabe JP, Rogers JM, Butler K, et al. Recovery of coordinated gait: randomized controlled stroke trial of functional electrical stimulation (FES) versus no FES, with weight-supported treadmill and over-ground training. Neurorehabilitation and Neural Repair 2011;25(7):588-96

\section{Dujović 2017 \{published data only\}}

Dujović SD, Malešević J, Maleševićc N, Vidaković AS Bijelić G, Keller T, et al. Novel multi-pad functional electrical stimulation in stroke patients: a single-blind randomized study. NeuroRehabilitation 2017;41(4):791-800.

\section{Embrey 2010 \{published data only\}}

Embrey DG, Holtz SL, Alon G, Brandsma BA, McCoy SW. Functional electrical stimulation to dorsiflexors and plantar flexors during gait to improve walking in adults with chronic hemiplegia. Archives of Physical Medicine and Rehabilitation 2010;91(5):687-96.

\section{Ernst 2013 \{published data only\}}

Ernst J, Grundey J, Hewitt M, Von LF, Kaus J, Schmalz T, et al. Towards physiological ankle movements with the ActiGait implantable drop foot stimulator in chronic stroke. Restorative Neurology and Neuroscience 2013;31(5):557-69.

\section{Everaert 2010 \{published data only\}}

Everaert DG, Thompson AK, Chong SL, Stein RB. Does functional electrical stimulation for foot drop strengthen corticospinal connections?. Neurorehabilitation and Neural Repair 2010;24(2):168-77.

\section{Everaert 2013 \{published data only\}}

Everaert DG, Stein RB, Abrams GM, Dromerick AW, Francisco GE, Hafner BJ, et al. Effect of a foot-drop stimulator and ankle-foot orthosis on walking performance after stroke: a multicenter randomized controlled trial. Neurorehabilitation and Neural Repair 2013;27(7):579-91.

\section{Fujiwara 2009 \{published data only\}}

Fujiwara T, Kasashima Y, Honaga K, Muraoka Y, Tsuji T, Osu R, et al. Motor improvement and corticospinal modulation induced by hybrid assistive neuromuscular dynamic stimulation (HANDS) therapy in patients with chronic stroke. Neurorehabilitation and Neural Repair 2009;23(2):125-32.

Gabr 2005 \{published data only\}

Gabr U, Levine P, Page SJ. Home-based electromyographytriggered stimulation in chronic stroke. Clinical Rehabilitation 2005;19(7):737-45.

\section{Ghédira 2017 \{published data only\}}

Ghédira M, Albertsen IM, Mardale V, Gracies JM, Bayle N, Hutin É. Wireless, accelerometry-triggered functional electrical stimulation of the peroneal nerve in spastic paresis: a randomized, controlled pilot study. Assistive Technology 2017;29(2):99-105.

\section{Granat 1996 \{published data only\}}

Granat MH, Maxwell DJ, Ferguson ACB, Lees KR, Barbenel JC. Peroneal stimulator: evaluation for the correction of spastic drop foot in hemiplegia. Archives of Physical Medicine and Rehabilitation 1996;77(1):19-24.

\section{Hara 2008 \{published data only\}}

Hara Y, Ogawa S, Tsujiuchi K, Muraoka Y. A home-based rehabilitation program for the hemiplegic upper extremity by power-assisted functional electrical stimulation. Disability and Rehabilitation 2008;30(4):296-304.

Hausdorff 2008 \{published data only\}

Hausdorff JM, Ring H. Effects of a new radio frequencycontrolled neuroprosthesis on gait symmetry and rhythmicity in 
patients with chronic hemiparesis. American Journal of Physical Medicine and Rehabilitation 2008;87(1):4-13.

\section{Jonsdottir 2017 \{published data only\}}

Jonsdottir J, Thorsen R, Aprile I, Galeri S, Spannocchi G, Beghi E, et al. Arm rehabilitation in post stroke subjects: a randomized controlled trial on the efficacy of myoelectrically driven FES applied in a task-oriented approach. PLOS ONE 2017;12(12):e0188642.

\section{Karniel 2019 \{published data only\}}

Karniel N, Raveh E, Schwartz I, Portnoy S. Functional electrical stimulation compared with ankle-foot orthosis in subacute post stroke patients with foot drop: a pilot study. Assistive Technology 2019. [DOI: 10.1080/10400435.2019.1579269]

\section{Kim 2016 \{published data only\}}

Kim S, Park J, Jung M, Yoo E. Effects of task-oriented training as an added treatment to electromyogram-triggered neuromuscular stimulation on upper extremity function in chronic stroke patients. Occupational Therapy International 2016;23(2):165-74

\section{Kimberley 2004 \{published data only\}}

Kimberley TJ, Lewis SM, Auerbach EJ, Dorsey LL, Lojovich JM, Carey JR. Electrical stimulation driving functional improvements and cortical changes in subjects with stroke. Experimental Brain Research 2004;154(4):450-60.

\section{Knutson 2012 \{published data only\}}

Knutson JS, Harley MY, Hisel TZ, Hogan SD, Maloney MM, Chae J. Contralaterally controlled functional electrical stimulation for upper extremity hemiplegia: an early-phase randomized clinical trial in subacute stroke patients. Neurorehabilitation and Neural Repair 2012;26(3):239-46.

\section{Kojovic 2009 \{published data only\}}

Kojovic J, Djuric-Jovicic M, Dosen S, Popovic MB, Popovic DB. Sensor-driven four-channel stimulation of paretic leg: functional electrical walking therapy. Journal of Neuroscience Methods 2009;181(1):100-5.

\section{Laufer 2009 \{published data only\}}

Laufer Y, Ring H, Sprecher E, Hausdorff JM. Gait in individuals with chronic hemiparesis: one-year follow-up of the effects of a neuroprosthesis that ameliorates foot drop. Journal of Neurologic Physical Therapy 2009;33:104-10.

\section{Mann 2011 \{published data only\}}

Mann G, Taylor P, Lane R. Accelerometer-triggered electrical stimulation for reach and grasp in chronic stroke patients: a pilot study. Neurorehabilitation and Neural Repair 2011;25(8):774-80

\section{Martin 2016 \{published data only\}}

Martin KD, Polanski WH, Schulz A, Jobges M, Hoff H, Schackert $\mathrm{G}$, et al. Restoration of ankle movements with the ActiGait implantable drop foot stimulator: a safe and reliable treatment option for permanent central leg palsy. Journal of Neurosurgery 2016;124(1):70-6.

\section{Marvulli 2016 \{published data only\}}

Marvulli R, Mastromauro L, Romanelli E, Lopopolo A, Dargenio M, Fomarelli F, et al. How botulinum toxin type Aoccupational therapy (OT)-functional electrical stimulation (FES) modify spasticity and functional recovery in patients with upper limb spasticity post stroke. Clinical Immunology, Endocrine \& Metabolic Drugs 2016;3(1):62-7.

\section{McCabe 2015 \{published data only\}}

McCabe J, Monkiewicz M, Holcomb J, Pundik S, Daly JJ. Comparison of robotics, functional electrical stimulation, and motor learning methods for treatment of persistent upper extremity dysfunction after stroke: a randomized controlled trial. Archives of Physical Medicine and Rehabilitation 2015;96(6):981-90.

\section{Morone 2012 \{published data only\}}

Morone G, Fusco A, Di Capua P, Coiro P, Pratesi L. Walking training with foot drop stimulator controlled by a tilt sensor to improve walking outcomes: a randomized controlled pilot study in patients with stroke in subacute phase. Stroke Research and Treatment 2012; Vol. 2012:Article ID 523564.

NCT03946488 \{published data only\} NCT03946488. Functional impact of a closed-loop controlled grasping neuroprosthesis in post-stroke patients (PREHENSTROKE). clinicaltrials.gov/ct2/show/NCT03946488 (first received 10 May 2019).

\section{NCT04014270 \{published data only\}}

NCT04014270. Self-modulated functional electrical stimulation in chronic stroke patients with severe and moderate upper limb paresis (SM-FES). clinicaltrials.gov/ct2/show/NCT04014270 (first received 10 July 2019).

\section{Ochi 2018 \{unpublished data only\}}

Ochi M, Kato N, Saeki S, Hachisuka K. Effects of peroneal nerve functional electrical stimulation (WalkAide ${ }^{\circledR}$ ) on lower extremities of patients with chronic stroke and hemiplegia - a multicenter, prospective, randomized controlled trial. Cerebrovascular Diseases 2018:45:440-1.

Page 2012 \{published data only\}

Page SJ, Levin L, Hermann V, Dunning K, Levine P. Longer versus shorter daily durations of electrical stimulation during taskspecific practice in moderately impaired stroke. Archives of Physical Medicine and Rehabilitation 2012;93(2):200-6.

\section{Popovic 2004a \{published data only\}}

Popovic DB, Popovic MB, Sinkjaer T, Stefanovic A, Schwirtlich L. Therapy of paretic arm in hemiplegic subjects augmented with a neural prosthesis: a cross-over study. Canadian Journal of Physiology and Pharmacology 2004;82(8-9):749-56.

\section{Popovic 2004b \{published data only\}}

Popovic MB, Popovic DB, Schwirtlich L, Sinkjaer T. Functional Electrical Therapy (FET): clinical trial in chronic hemiplegic subjects. Neuromodulation 2004;7(2):133-40. 
Popovic 2005 \{published data only\}

Popovic MR, Thrasher TA, Zivanovic V, Takaki J, Hajek V. Neuroprosthesis for retraining reaching and grasping functions in severe hemiplegic patients. Neuromodulation 2005;8(1):58-72.

\section{Qian 2017 \{published data only\}}

Qian Q, Hu X, Lai Q, Ng SC, Zheng Y, Poon W. Early stroke rehabilitation of the upper limb assisted with an electromyography-driven neuromuscular electrical stimulationrobotic arm. Frontiers in Neurology 2017;8:447. [DOI: 10.3389/ fneur.2017.00447]

\section{Ring 2005 \{published data only\}}

Ring H, Rosenthal N. Controlled study of neuroprosthetic functional electrical stimulation in sub-acute poststroke rehabilitation. Journal of Rehabilitation Medicine 2005;37(1):32-6.

\section{Ring 2009 \{published data only\}}

Ring H, Treger I, Gruendlinger L, Hausdorff JM. Neuroprosthesis for footdrop compared with an ankle-foot orthosis: effects on postural control during walking. Journal of Stroke and Cerebrovascular Diseases 2009;18(1):41-7.

\section{Sabut 2010 \{published data only\}}

Sabut SK, Sikdar C, Mondal R, Kumar R, Mahadevappa M. Restoration of gait and motor recovery by functional electrical stimulation therapy in persons with stroke. Disability and Rehabilitation 2010;32(19):1594-603.

\section{Salisbury 2013 \{published data only\}}

Salisbury L, Shiels J, Todd I, Dennis M. A feasibility study to investigate the clinical application of functional electrical stimulation (FES), for dropped foot, during the sub-acute phase of stroke-a randomized controlled trial. Physiotherapy Theory and Practice 2013;29(1):31-40.

\section{Sheffler 2006 \{published data only\}}

Sheffler LR, Hennessey MT, Naples GG, Chae J. Peroneal nerve stimulation versus an ankle foot orthosis for correction of footdrop in stroke: impact on functional ambulation. Neurorehabilitation and Neural Repair 2006;20(3):355-60.

\section{Sheffler 2013b \{published data only\}}

Sheffler LR, Nogan BS, Wilson RD, Chae J. Spatiotemporal, kinematic, and kinetic effects of a peroneal nerve stimulator versus an ankle foot orthosis in hemiparetic gait. Neurorehabilitation and Neural Repair 2013;27(5):403-10.

\section{Shindo 2017 \{published data only\}}

Shindo K, Fujiwara T, Hara J, Oba H, Hotta F, Tsuji T, et al. Effectiveness of hybrid assistive neuromuscular dynamic stimulation therapy in patients with subacute stroke: a randomized controlled pilot trial. Neurorehabilitation and Neural Repair 2017;25(9):830-7.

\section{Singer 2013 \{published data only\}}

Singer BJ, Vallence A, Cleary S, Cooper I, Loftus AM. The effect of EMG triggered electrical stimulation plus task practice on arm function in chronic stroke patients with moderate- severe arm deficits. Restorative Neurology and Neuroscience 2013;31(6):681-91.

Taylor 1999 \{published data only\}

Taylor PN, Burridge JH, Dunkerley AL, Wood DE, Norton JA, Singleton $\mathrm{C}$, et al. Clinical use of the Odstock dropped foot stimulator: its effect on the speed and effort of walking. Archives of Physical Medicine and Rehabilitation 1999;80(12):1577-83.

Taylor 2013 \{published data only\}

Taylor P, Humphreys L, Swain I. The long-term costeffectiveness of the use of functional electrical stimulation for the correction of dropped foot due to upper motor neuron lesion. Journal of Rehabilitation Medicine 2013;45(2):154-60.

Thorsen 2013 \{published data only\}

Thorsen R, Cortesi M, Jonsdottir J, Carpinella I, Morelli D, Casiraghi A, et al. Myoelectrically driven functional electrical stimulation may increase motor recovery of upper limb in poststroke subjects: a randomized controlled pilot study. Journal of Rehabilitation Research and Development 2013;50(6):785-94.

Turk 2008 \{published data only\}

Turk R, Burridge JH, Davis R, Cosendai G, Sparrow O, Roberts HC, et al. Therapeutic effectiveness of electric stimulation of the upper-limb poststroke using implanted microstimulators. Archives of Physical Medicine and Rehabilitation 2008;89(10):1913-22.

\section{UMIN000026624 \{published data only\}}

UMIN000026624. Clinical application of a wearable integrated volitional control electrical stimulation device for patient with motor disability in upper extremity at subacute phase. upload.umin.ac.jp/cgi-open-bin/ctr_e/ctr_view.cgi? recptno=R000030533 (first received 1 April 2017).

\section{Van Swigchem 2012 \{published data only\}}

van Swigchem R, van Duijnhoven HJR, den Boer J, Geurts AC, Weerdesteyn V. Effect of peroneal electrical stimulation versus an ankle-foot orthosis on obstacle avoidance ability in people with stroke-related foot drop. Physical Therapy 2012;92(3):398-406.

\section{Varkuti 2013 \{published data only\}}

Varkuti B, Guan C, Pan Y, Phua KS, Ang KK, Kuah CWK, et al. Resting state changes in functional connectivity correlate with movement recovery for $\mathrm{BCl}$ and robot-assisted upper-extremity training after stroke. Neurorehabilitation and Neural Repair 2013;27(1):53-62.

\section{Veltink 2003 \{published data only\}}

Veltink PH, Slycke P, Hemssems J, Buschman R, Bultstra G, Hermens $\mathrm{H}$. Three dimensional inertial sensing of foot movements for automatic tuning of a two-channel implantable drop-foot stimulator. Medical Engineering and Physics 2003;25(1):21-8.

\section{Von Lewinski 2009 \{published data only\}}

Von Lewinski F, Hofer S, Kaus J, Merboldt K, Rothkegel H, Schweizer R, et al. Efficacy of EMG-triggered electrical arm 
stimulation in chronic hemiparetic stroke patients. Restorative Neurology and Neuroscience 2009;27(3):189-97.

Wilkinson 2015 \{published data only\}

Wilkinson IA, Burridge J, Strike P, Taylor P. A randomised controlled trial of integrated electrical stimulation and physiotherapy to improve mobility for people less than 6 months post stroke. Disability \& Rehabilitation: Assistive Technology 2015;10(6):468-74.

Yao 2017 \{published data only\}

Yao D, Lahner M, Jakubowitz E, Thomann A, Ettinger S, Noll Y, et al. Hip and knee effects after implantation of a drop foot stimulator. Technology and Health Care 2017;25(3):599-606.

\section{References to studies awaiting assessment}

\section{ISRCTN91639560 \{published data only\}}

ISRCTN91639560. Pilot study to investigate the effectiveness of combining physiotherapy and electrical stimulation to improve mobility in recently discharged stroke patients. www.isrctn.com/ISRCTN91639560 (first received 9 March 2007).

NCT03574623 \{published data only\}

NCT03574623. Therapies for recovery of hand function after stroke. clinicaltrials.gov/ct2/show/NCT03574623 (first received 2 July 2018).

\section{UMIN000018648 \{published data only\}}

UMIN000018648. The effect of HANDS therapy among outpatients with chronic hemiparesis: randomized control study. upload.umin.ac.jp/cgi-open-bin/ctr_e/ctr_view.cgi? recptno=R000021593 (first received 13 August 2015).

Wright 2004 \{published data only\}

Wright PA, Mann G, Swain I. A comparison of electrical stimulation and the conventional ankle foot orthosis in the correction of a dropped foot following stroke. 9th Annual Conference of the International FES Society; 2004 Sep 6-9; Bournemouth (UK). https://www.odstockmedical.com/sites/ default/files/wrightp1.pdf, 2004.

\section{References to ongoing studies}

Ghedira 2014 \{published data only (unpublished sought but not used)\}

Ghedira M, Hutin E, Albertsen IM, Bayle N, Gracies JM, Decq P. Randomized controlled trial comparing implanted peroneal nerve stimulation and ankle foot orthosis in spastic paresis. Annals of Physical and Rehabilitation Medicine 2014;57 Suppl 1:e19-20.

\section{Additional references}

\section{Alon 2003b}

Alon G. Use of neuromuscular electrical stimulation in neurorehabilitation: a challenge to all. Journal of Rehabilitation Research and Development 2003;40:ix-xii.

\section{Ambrosini 2011}

Ambrosini E, Ferrante S, Pedrocchi A, Ferrigno G, Molteni F. Cycling induced by electrical stimulation improves motor recovery in postacute hemiparetic patients: a randomized controlled trial. Stroke 2011;42(4):1068-73.

\section{Ambrosini 2014}

Ambrosini E, Bejarano NC, Pedrocchi A. Sensors for motor neuroprosthetics: current applications and future directions. In: Naik GR, Guo Y editor(s). Emerging Theory and Practice in Neuroprosthetics. Hershey (USA): IGI Global, 2014.

\section{Anderson 1996}

Anderson C, Laubscher S, Burns R. Validation of the Short Form 36 (SF-36) health survey questionnaire among stroke patients. Stroke 1996;27(10):1812-6.

\section{Atkins 2004}

Atkins D, Best D, Briss PA, Eccles M, Falck-Ytter Y, Flottorp S, et al. GRADE Working Group. Grading quality of evidence and strength of recommendations. BMJ 2004;328(7454):1490.

\section{Auchstaetter 2016}

Auchstaetter N, Luc J, Lukye S, Lynd K, Schemenauer S, Whittaker M, et al. Physical therapists' use of functional electrical stimulation for clients with stroke: frequency, barriers, and facilitators. Physical Therapy 2016;96:995-1005.

\section{Baer 2001}

Baer HR, Wolf SL. Modified memory functional ambulation profile: an outcome measure for the rehabilitation of poststroke gait dysfunction. Stroke 2001;32(4):973-9.

\section{Berg 1992}

Berg KO, Wood-Dauphinee SL, Williams JI, Maki B. Measuring balance in the elderly: validation of an instrument. Canadian Journal of Public Health 1992;83 Suppl 2:S7-11.

\section{Bethoux 2015}

Bethoux F, Rogers HL, Nolan KJ, Abrams GM, Annaswamy T, Brandstater $\mathrm{M}$, et al. Long-term follow-up to a randomized controlled trial comparing peroneal nerve functional electrical stimulation to an ankle foot orthosis for patients with chronic stroke. Neurorehabilitation and Neural Repair 2015;29(10):911-22.

\section{Bolton 2004}

Bolton DAE, Cauraugh JH, Hausenblas HA. Electromyogramtriggered neuromuscular stimulation and stroke motor recovery of arm/hand functions: a meta-analysis. Journal of the Neurological Sciences 2004;223(2):121-7.

\section{Borges 2018}

Borges LRDM, Fernandes ABGS, Melo LP, Guerra RO, Campos TF. Action observation for upper limb rehabilitation after stroke. Cochrane Database of Systematic Reviews 2018, Issue 10. [DOI: 10.1002/14651858.CD011887.pub2]

\section{Bosch 2014}

Bosch PR, Harris JE, Wing K. Review of therapeutic electrical stimulation for dorsiflexion assist and orthotic substitution 
from the American Congress of Rehabilitation Medicine Stroke Movement Interventions Subcommittee. Archives of Physical Medicine and Rehabilitation 2014;95(2):390-6.

\section{Brehm 2011}

Brehm M, Bus SA, Harlaar J, Nollet F. A candidate core set of outcome measures based on the international classification of functioning, disability and health for clinical studies on lower limb orthoses. Prosthetics and Orthotics International 2011;35(3):269-77.

\section{Cambridge 1977}

Cambridge NA. Electrical apparatus used in medicine before 1900. Proceedings of the Royal Society of Medicine 1977;70:635-41.

\section{Collen 1991}

Collen FM, Wade DT, Robb GF, Bradshaw CM. The Rivermead Mobility Index: a further development of the Rivermead Motor Assessment. International Disability Studies 1991;13(2):50-4.

\section{Collinger 2013}

Collinger JL, Foldes S, Bruns TM, Wodlinger B, Gaunt R, Weber DJ. Neuroprosthetic technology for individuals with spinal cord injury. Journal of Spinal Cord Medicine 2013;36(4):258-72.

\section{Covidence [Computer program]}

Veritas Health Innovation. Covidence. Version accessed 15 November 2019. Melbourne, Australia: Veritas Health Innovation.

\section{Cowan 2012}

Cowan RE, Fregly BJ, Boninger ML, Chan L, Rodgers MM, Reinkensmeyer DJ. Recent trends in assistive technology for mobility. Journal of NeuroEngineering and Rehabilitation 2012;9:20.

\section{de Campos 2017}

de Campos LM, Martins BM, Cabral NL, Franco SC, PontesNeto OM, Mazin SC, et al. How many patients become functionally dependent after a stroke? A 3-year population based study in Joinville, Brazil. PLOS ONE 2017;12(1):e0170204.

\section{Dean 1992}

Dean C, Mackey F. Motor assessment scale scores as a measure of rehabilitation outcome following stroke. Australian Journal of Physiotherapy 1992;38(1):31-5.

\section{Duncan 1999}

Duncan PW, Wallace D, Lai SM, Johnson D, Embretson S, Laster LJ. The stroke impact scale version 2.0. Evaluation of reliability, validity, and sensitivity to change. Stroke 1999;30(10):2131-40.

\section{Dunning 2013}

Dunning K, O’Dell M, Kluding P, Wu S, Feld J, Ginosian J, et al. The functional ambulation: standard treatment vs. electrical stimulation therapy (fastest) trial for stroke: study design and protocol. Open Access Journal of Clinical Trials 2013;5:39-49.

\section{Dunning 2015}

Dunning KO, Dell MW, Kluding P, McBride K. Peroneal stimulation for foot drop after stroke: a systematic review. American Journal of Physical Medicine and Rehabilitation 2015;94(8):649-64.

\section{Eng 2007}

Eng JJ, Tang PF. Gait training strategies to optimize walking ability in people with stroke: a synthesis of the evidence. Expert Review of Neurotherapeutics 2007;7(10):1417-36.

\section{Feigin 2014}

Feigin VL, Forouzanfar MH, Krishnamurthi R, Mensah GA, Connor M, Bennett DA, et al. Global and regional burden of stroke during 1990-2010: findings from the Global Burden of Disease Study 2010. Lancet 2014;383(9913):245-55.

\section{Fulk 2018}

Fulk GD, He Y. Minimal clinically important difference of the 6Minute Walk Test in people with stroke. Journal of Neurologic Physical Therapy 2018;42(4):235-40.

\section{GRADEpro GDT [Computer program]}

McMaster University (developed by Evidence Prime). GRADEpro GDT. Version accessed 15 November 2019. Hamilton (ON): McMaster University (developed by Evidence Prime), 2015.

\section{Grey Matters}

Canadian Agency for Drugs and Technologies in Health (CADTH) Research Information Services. Grey Matters: a practical tool for searching health-related grey literature. www.cadth.ca/ resources/finding-evidence/grey-matters (accessed 19 August 2019).

\section{Hamilton 1994}

Hamilton BB, Laughlin JA, Fiedler RC, Granger CV. Interrater reliability of the 7-level functional independence measure (FIM). Scandinavian Journal of Rehabilitation Medicine 1994;26(3):115-9.

\section{Heller 1987}

Heller A, Wade DT, Wood VA, Sunderland A, Hewer RL, Ward E. Arm function after stroke: measurements and recovery over the first three months. Journal of Neurology, Neurosurgery, and Psychiatry 1987;50:714-9.

\section{Hiengkaew 2012}

Hiengkaew V, Jitaree $\mathrm{K}$, Chaiyawat P. Minimal detectable changes of the Berg Balance Scale, Fugl-Meyer Assessment Scale, Timed "Up \& Go" Test, gait speeds, and 2-minute walk test in individuals with chronic stroke with different degrees of ankle plantarflexor tone. Archives of Physical Medicine and Rehabilitation 2012;93:1201-8.

\section{Higgins 2011a}

Higgins JP, Deeks JJ, editor(s). Chapter 7: Selecting studies and collecting data. In: Higgins JP, Green S, editor(s). Cochrane Handbook for Systematic Reviews of Interventions Version 5.1.0 (updated March 2011). The Cochrane Collaboration, 2011. Available from handbook.cochrane.org. 


\section{Higgins 2011b}

Higgins JP, Altman DG, Sterne JA, editor(s). Chapter 8: Assessing risk of bias in included studies. In: Higgins JP, Green S, editor(s). Cochrane Handbook for Systematic Reviews of Interventions Version 5.1.0 (updated March 2011). The Cochrane Collaboration, 2011. Available from handbook.cochrane.org.

\section{Higgins 2011c}

Deeks JJ, Higgins JPT, Altman DG, editor(s). Chapter 9: Analysing data and undertaking meta-analyses. In: Higgins JP, Green S, editor(s). Cochrane Handbook for Systematic Reviews of Interventions Version 5.1.0 (updated March 2011). The Cochrane Collaboration, 2011. Available from handbook.cochrane.org.

\section{Holden 1984}

Holden MK, Gill KM, Magliozzi MR, Nathan J, Piehl-Baker L. Clinical gait assessment in the neurologically impaired. Reliability and meaningfulness. Physical Therapy 1984;64:35-40.

\section{Horch 2004}

Horch KW, Dhillon GS, editor(s). Neuroprosthetics: Theory and Practice. Vol. 2, Singapore: World Scientific Publishing Co, 2004.

\section{Jonsdottir 2007}

Jonsdottir J, Cattaneo D. Reliability and validity of the dynamic gait index in persons with chronic stroke. Archives of Physical Medicine and Rehabilitation 2007;88(11):1410-5.

\section{Kluding 2014}

Kluding PM, Dunning K, O'Dell MW, Wu SS, Ginosian J, Feld J, et al. Foot drop stimulation versus ankle foot orthosis after stroke. Stroke 2014;44:1660-9.

\section{Kottink 2004}

Kottink Al, Oostendorp LJ, Buurke JH, Nene AV, Hermens HJ, IJzerman MJ. The orthotic effect of functional electrical stimulation on the improvement of walking in stroke patients with a dropped foot: a systematic review. Artificial Organs 2004;28(6):577-86.

\section{Kottink 2008}

Kottink AIR, Hermens HJ, Nene AV, Tenniglo MJ, GroothuisOudshoorn CG, IJzerman MJ. Therapeutic effect of an implantable peroneal nerve stimulator in subjects with chronic stroke and footdrop: a randomized controlled trial. Physical Therapy 2008;88(4):437-48.

\section{Kottink 2010}

Kottink Al, IJzerman MJ, Groothuis-Oudshoorn CG, Hermens HJ. Measuring quality of life in stroke subjects receiving an implanted neural prosthesis for drop foot. Artificial Organs 2010;34(5):366-76.

\section{Kottink 2012}

Kottink Al, Tenniglo MJ, de Vries $\mathrm{WH}$, Hermens $\mathrm{HJ}$, Buurke JH. Effects of an implantable two-channel peroneal nerve stimulator versus conventional walking device on spatiotemporal parameters and kinematics of hemiparetic gait. Journal of Rehabilitation Medicine 2012;44:51-7.

\section{Lang 2013}

Lang CE, Bland MD, Bailey RR, Schaefer SY, Birkenmeier RL. Assessment of upper extremity impairment, function, and activity following stroke: foundations for clinical decision making. Journal of Hand Therapy 2013;26(2):104-15.

\section{Langhorne 2009}

Langhorne P, Coupar F, Pollock A. Motor recovery after stroke: a systematic review. Lancet Neurology 2009;8(8):741-54.

\section{Laver 2017}

Laver KE, Lange B, George S, Deutsch JE, Saposnik G, Crotty M. Virtual reality for stroke rehabilitation. Cochrane Database of Systematic Reviews 2017, Issue 11. [DOI: 10.1002/14651858.CD008349.pub4]

\section{Lefebvre 2011}

Lefebvre C, Manheimer E, Glanville J. Chapter 6: Searching for studies. In: Higgins JP, Green S, editor(s). Cochrane Handbook for Systematic Reviews of Interventions Version 5.1.0 (updated March 2011). The Cochrane Collaboration, 2011. Available from handbook.cochrane.org.

\section{Liberati 2009}

Liberati A, Altman DG, Tetzlaff J, Mulrow C, Gotzsche PC, Ioannidis JP, et al. The PRISMA statement for reporting systematic reviews and meta-analyses of studies that evaluate health care interventions: explanation and elaboration. PLOS Medicine 2009;6(7):e1000100.

\section{Liberson 1961}

Liberson WT, Holmquest HJ, Scot D, Dow M. Functional electrotherapy: stimulation of the peroneal nerve synchronized with the swing phase of the gait of hemiplegic patients. Archives of Physical Medicine and Rehabilitation 1961;42:101-5.

\section{Martin 2012}

Martin R, Sadowsky C, Obst K, Meyer B, McDonald J. Functional electrical stimulation in spinal cord injury: from theory to practice. Topics in Spinal Cord Injury Rehabilitation 2012;18(1):28-33.

\section{Martins 2012}

Martins EF, de Menezes LT, de Sousa PH, de Araujo Barbosa PH, Costa AS. Reliability of the Functional Reach Test and the influence of anthropometric characteristics on test results in subjects with hemiparesis. NeuroRehabilitation 2012;31(2):161-9.

\section{Mathiowetz 1985}

Mathiowetz V, Volland G, Kashman N, Weber K. Adult norms for the box and block test of manual dexterity. American Journal of Occupational Therapy 1985;39(6):386-91.

\section{Meilink 2008}

Meilink A, Hemmen B, Seelen HA, Kwakkel G. Impact of EMGtriggered neuromuscular stimulation of the wrist and finger extensors of the paretic hand after stroke: a systematic review of the literature. Clinical Rehabilitation 2008;22(4):291-305. 


\section{Melo 2015}

Melo PL, Silva MT, Martins JM, Newman DJ. Technical developments of functional electrical stimulation to correct drop foot: sensing, actuation and control strategies. Clinical Biomechanics 2015;30(2):101-13.

\section{Moss 2011}

Moss CW, Kilgore KL, Peckham PH. A novel command signal for motor neuroprosthetic control. Neurorehabilitation and Neural Repair 2011;25(9):847-54.

\section{Mudge 2007}

Mudge S, Stott NS. Outcome measures to assess walking ability following stroke: a systematic review of the literature. Physiotherapy 2007;93:189-200.

\section{Naik 2014}

Naik GR, Guo Y. Emerging Theory and Practice in Neuroprosthetics. Hershey (USA): IGI Global, 2014.

\section{NICE 2009}

National Institute for Health and Clinical Excellence (NICE). NICE interventional procedure guidance (IPG 278). Functional electrical stimulation for drop foot of central neurological origin. www.nice.org.uk/guidance/ipg278.

\section{Podsiadlo 1991}

Podsiadlo D, Richardson S. The timed "up \& go": a test of basic functional mobility for frail elderly persons. Journal of the American Geriatrics Society 1991;14(2):142-8.

\section{Popović 2014}

Popović DB. Advances in functional electrical stimulation (FES). Journal of Electromyography and Kinesiology 2014;24(6):795-802.

\section{Prenton 2016}

Prenton S, Hollands KL, Kenney LPJ. Functional electrical stimulation versus ankle foot orthoses for foot-drop: a metaanalysis of orthotic effects. Journal of Rehabilitation Medicine 2016;48:646-56.

\section{Quinn 2011}

Quinn TJ, Langhorne P, Stott DJ. Barthel index for stroke trials development, properties, and application. Stroke 2011;42(4):1146-51.

\section{Review Manager 2014 [Computer program]}

Nordic Cochrane Centre, The Cochrane Collaboration. Review Manager 5 (RevMan 5). Version 5.3. Copenhagen: Nordic Cochrane Centre, The Cochrane Collaboration, 2014.

\section{Roth 2017}

Roth GA, Johnson C, Abajobir A, Abd-Allah F, Abera SF, Abyu G, et al. Global, regional, and national burden of cardiovascular diseases for 10 causes, 1990 to 2015. Journal of the American College of Cardiology 2017;70(1):1-25.

\section{Schiemanck 2006}

Schiemanck SK, Kwakkel G, Marcel WM, Kappelle LJ, Prevo AJH. Predicting long-term independency in activities of daily living after middle cerebral artery stroke: does information from MRI have added predictive value compared with clinical information?. Stroke 2006;37(4):1050-4.

\section{Seale 2006}

Seale H. Six minute walking test. Australian Journal of Physiotherapy 2006;52(3):228.

\section{Sheffler 2007}

Sheffler LR, Chae J. Neuromuscular electrical stimulation in neurorehabilitation. Muscle and Nerve 2007;35:562-90.

\section{Sheffler 2009}

Sheffler LR, Hennessey MT, Knutson JS, Chae J. Neuroprosthetic effect of peroneal nerve stimulation in multiple sclerosis: a preliminary study. Archives of Physical Medicine and Rehabilitation 2009;90(2):362-5.

\section{Sheffler 2015}

Sheffler LR, Taylor PN, Bailey SN, Gunzler DD, Buurke JH, IJzerman MJ, et al. Surface peroneal nerve stimulation in lower limb hemiparesis: effect on quantitative gait parameters. American Journal of Physical Medicine and Rehabilitation 2015;94(5):341-57.

\section{Stein 2008}

Stein RB, Rolf R, Everaert DG, Bobet J, Chong SL. Surface electrical stimulation for foot drop: control aspects and walking performance. Journal of Automatic Control 2008;18(2):47-52.

\section{Stern 1992}

Stern EB. Stability of the Jebsen-Taylor hand function test across three test sessions. American Journal of Occupational Therapy 1992;46(7):647-9.

\section{Sullivan 2013}

Sullivan JE, Crowner BE, Kluding PM, Nichols D, Rose DK, Yoshida R, et al. Outcome measures for individuals with stroke: process and recommendations from the American Physical Therapy Association Neurology Section Task Force. Physical Therapy 2013;93:1383-96.

\section{Thibaut 2017}

Thibaut A, Moissenet F, Di Perri C, Schreiber C, Remacle A, Kolanowski $\mathrm{E}$, et al. Brain plasticity after implanted peroneal nerve electrical stimulation to improve gait in chronic stroke patients: two case reports. NeuroRehabilitation 2017;40(2):251-8.

\section{Thieme 2018}

Thieme H, Morkisch N, Mehrholz J, Pohl M, Behrens J, Borgetto $\mathrm{B}$, et al. Mirror therapy for improving motor function after stroke. Cochrane Database of Systematic Reviews 2018, Issue 7. [DOI: 10.1002/14651858.CD008449.pub3]

\section{Uswatte 2005}

Uswatte G, Taub E, Morris D, Vignolo M, McCulloch K. Reliability and validity of the upper-extremity motor activity log-14 for measuring real-world arm use. Stroke 2005;36:2493-6. 


\section{Watson 2002}

Watson MJ. Refining the ten-metre walking test for use with neurologically impaired people. Physiotherapy 2002;88(7):386-97.

\section{WHO 1989}

World Health Organization. Recommendations on stroke prevention, diagnosis, and therapy. Report of the WHO Task Force on Stroke and other Cerebrovascular Disorders. Stroke 1989;20(10):1407-31.

\section{WHO 2001}

World Health Organization. International Classification of Functioning, Disability and Health. Geneva: World Health Organization, 2001.

\section{Williams 1999}

Williams LS, Weinberger M, Harris LE, Clark DO, Biller J. Development of a Stroke-Specific Quality of Life Scale. Stroke 1999;30(7):1362-9.

\section{Wolf 1979}

Wolf SL. A method for quantifying ambulatory activities. Physical Therapy Journal 1979;59(6):767-8.

\section{Wolf 2001}

Wolf SL, Catlin PA, Ellis M, Archer AL, Morgan B, Piacentino A. Assessing Wolf Motor Function Test as outcome measure for research in patients after stroke. Stroke 2001;32:1635-9.

\section{Ziat 2015}

Ziat M, Bensmaia S. Neuroprosthetics. In: Wright JD editor(s). International Encyclopedia of the Social and Behavioral Sciences. 2nd Edition. Oxford: Elsevier Ltd, 2015.

\section{References to other published versions of this review Mendes 2018}

Mendes LA, Lima INDF, Souza T, do Nascimento GC, Resqueti VR, Fregonezi GAF. Motor neuroprosthesis for promoting recovery of function after stroke. Cochrane Database of Systematic Reviews 2018, Issue 3. [DOI: 10.1002/14651858.CD012991]

* Indicates the major publication for the study

\section{CHARACTERISTICS OF STUDIES}

Characteristics of included studies [ordered by study ID]

\section{Bethoux 2014}

Methods Study design: RCT

Instruments used: MMSE, BDI, 10MWT, SIS, device-related SAE rate, 6MWT, GaitRite FAP, mEFAP, BBS, TUG, SSQoL

Study design as described in the article: Quote: "This study was an unblinded, parallel-group RCT"

Study duration: 24 months

Year of study: trial ran between April 2010 and April 2012

Participants Inclusion criteria: $\geq 6$ months poststroke; inadequate dorsiflexion with inadequate limb clearance dur-
ing swing phase of gait; positive response to peroneal nerve stimulation testing; adequate cognitive
function (MMSE score $>17$ ); not currently using FES for the treatment of foot drop; $\geq 30$ days post-in-
patient or outpatient stroke, cardiac, pulmonary, or any other lower extremity physical rehabilitation;
able to walk at least 10 meters with or without an assist device; initial gait speed of $>0.0 \mathrm{~m} / \mathrm{s}$ and $<0.8$
$\mathrm{~m} / \mathrm{s}$; eligible for Medicare or Medicare Choice/Advantage benefits at time of consent; $\geq 90$ days post-MI;
$\geq 90$ days post-stenting procedure (i.e. peripheral, cardiac, carotid, and/or renal); $\geq 90$ days post-major
orthopedic surgery (i.e. hip, knee, and/or ankle joint replacement); $\geq 6$ months post-CABG or cardiac
valve procedure; able and willing to give written consent and comply with study procedures, including
follow-up visits
Exclusion criteria: ankle joint instability other than foot drop; needs AFO for stance control of the foot,
ankle, and/or knee; unable to safely clear toes in swing phase on the involved lower extremity, defined
as >-5 degrees plantar flexion with the WalkAide device (determined at fitting); diagnosed with periph-
eral neuropathy, and symptoms obstruct or limit ambulation or participation in study; diagnosed with
significant peripheral vascular disease accompanied by lower extremity ulceration and/or disabling
claudication; underlying condition(s) that would limit study participation; severe hypertonicity result-
ing in the need for more involved orthotic strategies; excessive dysesthetic pain secondary to neuro-
logical involvement; moderate to very severe chronic obstructive pulmonary disease, as defined by the
Global Initiative for Chronic Obstructive Lung Disease (GOLD); New York Heart Association (NYHA) Class 
III-IV; malignant skin lesion below the knee on the affected lower extremity; history of seizure disorder and is currently on seizure control medication for this disorder; aphasia, defined as inability to verbalize commands; BDI score of > 29 indicating severe depression; life expectancy less than 12 months; received botulinum toxin injections in the lower extremity within the past 6 months; baclofen pump with unstable dosing in the last 3 months; participating in another clinical trial that, according to the principal investigator, is likely to affect study outcome or confound results; patient has existing electrical stimulation devices (implantable cardioverter defibrillator, pacemaker, spinal stimulation, TENS)

Age: $M N$ group mean age $( \pm S D)$ : 63.87 years $( \pm 11.33)$; control group mean age $( \pm S D): 64.30$ years ( \pm 12.01)

Country: USA

Sample size: 495 participants

Sex: MN group: 147 (60.74\%) men, 95 (39.26\%) women; control group: 157 (62.06\%) men, 96 (37.94\%) women

Time poststroke: $\geq 6$ months poststroke. MN group mean time poststroke $( \pm S D): 6.90$ years $( \pm 6.43)$; control group mean time poststroke $( \pm S D): 6.86$ years $( \pm 6.64)$

Type of stroke: not stated

Interventions

Motor neuroprosthesis

- Intervention: MN group used WalkAide device for all walking activities on a full-time basis throughout the day. In the first 2 weeks, participants adhered to a progressive wearing schedule, after that they were instructed to wear $\mathrm{MN}$ on a full-time basis (i.e. for all walking activities throughout the day).

- Number of participants: 242

- Device: a single-channel electrical stimulator composed of a cuff worn around the proximal part of the lower leg, control module, and surface electrodes. This device uses a tilt sensor and accelerometer to trigger ankle dorsiflexion during the swing phase of gait.

- Duration of exposure: the length of treatment with MN was 12 months

- Place of application of intervention: lower limb

Another assistive technology device

- Intervention: control group used AFO for all walking activities on a full-time basis throughout the day. In the first 2 weeks, participants adhered to a progressive wearing schedule, after that they were instructed to wear AFO on a full-time basis (i.e. for all walking activities throughout the day).

- Number of participants: 253

- Device: AFO could be either articulated or fixed at the ankle based on the professional opinion of the orthotist and clinical needs of the participant

- Duration of exposure: the length of treatment with AFO was 12 months

- Place of application of intervention: lower limb

- Outcome type: continuous

- Assessment time point: baseline, 6 months, and 12 months

- Device at assessments: baseline, 6-month, and 12-month assessments performed with MN

Activities involving limbs: mEFAP (s)

- Outcome type: continuous

- Assessment time point: baseline, 6 months, and 12 months

- Device at assessments: baseline, 6-month, and 12-month assessments performed with MN

Activities involving limbs: TUG (s)

- Outcome type: continuous 
Bethoux 2014 (Continued)

- Assessment time point: baseline and 6 months

- Device at assessments: baseline, 6-month, and 12-month assessments performed with MN

Balance: BBS

- Outcome type: continuous

- Assessment time point: baseline and 6 months

- Device at assessments: baseline, 6-month, and 12-month assessments performed with MN

Exercise capacity: 6MWT (m)

- Outcome type: continuous

- Assessment time point: baseline, 6 months, and 12 months

- Device at assessments: baseline, 6-month, and 12-month assessments performed with MN

Participation scale of HRQoL: SSQoL

- Outcome type: continuous

- Assessment time point: baseline and 6 months

Participation scale of HRQoL: SIS Social participation domain

- Outcome type: continuous

- Assessment time point: baseline and 6 months

Adverse events: dropouts during the intervention period

- Outcome type: binary

Adverse events: serious adverse events related to the intervention

- Outcome type: binary

Adverse events: falls

- Outcome type: binary

Author's name: Francois Bethoux

Institution: The Cleveland Clinic Foundation

Email: bethouf@ccf.org

Address: The Cleveland Clinic Foundation, Desk U10, 9500 Euclid Avenue, Cleveland, OH 44195, USA

\begin{tabular}{ll}
\hline Funding source & Innovative Neurotronics \\
\hline Notes & This study consisted of 2 articles (Bethoux 2014; Bethoux 2015).
\end{tabular}

\section{Risk of bias}

\begin{tabular}{lll}
\hline Bias & Authors' judgement & Support for judgement \\
\hline $\begin{array}{l}\text { Random sequence genera- } \\
\text { tion (selection bias) }\end{array}$ & Low risk & $\begin{array}{l}\text { Quote: "Using centralized computer-generated randomization scheme built } \\
\text { into the electronic data capture system for this study" }\end{array}$ \\
\hline $\begin{array}{l}\text { Allocation concealment } \\
\text { (selection bias) }\end{array}$ & Low risk & Quote: "Centralized computer-generated randomization scheme" \\
\hline $\begin{array}{l}\text { Blinding of participants } \\
\text { and personnel (perfor- } \\
\text { mance bias) }\end{array}$ & High risk & There was no blinding of participants and personnel. \\
\hline
\end{tabular}


Bethoux 2014 (Continued)

All outcomes

Blinding of outcome as-
sessment (detection bias) $\quad$ High risk Unblinded outcome assessment

sessment (detection bias)

All outcomes

$\begin{array}{ll}\begin{array}{l}\text { Incomplete outcome data } \\ \text { (attrition bias) }\end{array} & \text { Low risk } \\ \text { Secondary outcome } & \text { for missing data" }\end{array}$

Secondary outcome

Incomplete outcome data Low risk

(attrition bias)

Primary outcome

Selective reporting (re- Unclear risk

porting bias)
Quote: "We conducted an ITT analysis using multiple imputations to account for missing data"

Other bias High risk This study was sponsored by Innovative Neurotronics.

Kluding 2013

Study design: RCT
Instruments used: 10MWT, lower extremity Fugl-Meyer, TUG, 6MWT, BBS, FRT, SIS
Study design as described in the article: "single-blinded randomized controlled trial"
Study duration: 32 months
Year of study: trial ran between May 2010 and December 2012

Participants

Inclusion criteria: at least 1 stroke $\geq 3$ months before study enrollment, resulting in drop foot; ankle dorsiflexion response with test stimulation in sitting and standing, and adequate ankle and knee stability during gait with test stimulation; medically stable; score $\geq 24$ on the MMSE, or have a competent caregiver if <24; age $\geq 18$ year or older; able to walk $\geq 10$ meters with a maximum of 1 person assist; self-selected gait speed $\leq 0.80 \mathrm{~m} / \mathrm{s}$ without orthotic effect

Exclusion criteria: fixed ankle contracture at $\geq 5$ degrees of plantar flexion in the hemiplegic leg with the knee extended; pain in the affected leg, rated $\geq 4$ on a 10-point visual analogue scale; participating in physical therapy, occupational therapy, new exercise program, or any other interventional clinical research studies without the sponsor's approval; botulinum toxin to the hemiplegic leg or arm within the past 6 weeks or planned during the course of the study; expectation of a significant change in oral medications for spasticity; complete lower extremity hemisensory loss; use of any FDS device for foot drop for an accumulative $>3$ hours within the last 6 months before study enrollment; any electric or metallic implant; significant swelling/edema in the lower leg; chronic skin problems or cancerous lesion in close proximity to the site of FDS stimulation; pregnant or planning on becoming pregnant; unstable seizure disorder; orthopedic conditions that would affect ambulation; major untreated depression

Sample size: 197 participants

Country: USA

Age: mean age $( \pm S D): 61.14$ years $( \pm 11.61)$

Sex: 79 women and 118 men. MN group: 51 (51.5\%) men; control group: 67 (68.4\%) men

Time poststroke: this study considered 2 subgroups: participants 3 to 6 months after stroke and participants $>6$ months after stroke. Mean time poststroke $( \pm S D): 4.55$ years $( \pm 4.72)$ 
- Intervention: MN group used NESS L300 device. In the first 6 weeks, participants received 8 physical therapy sessions and also followed the device manufacturer's standard conditioning protocol. The physical therapy sessions focused on education on the MN device use, gait training with $\mathrm{MN}$, and the development of an individualized home exercise program. Treatment time ranged from 30 to 60 minutes. The standard conditioning protocol performed in the first 3 weeks included the gradual increase of walking with MN from 15 minutes each day to all-day use and also involved the use of the device for cyclic stimulation while the participant was not walking. Participants performed cyclic stimulation to gradually strengthen and condition the muscles to avoid fatigue when using the MN. This stimulation was done 2 times daily for 15 minutes in the first week and for 20 minutes over the next 2 weeks. After this initial conditioning phase, participants used $\mathrm{MN}$ all day exclusively for ambulation.

- Number of participants: 99

- Device: a single-channel electrical stimulator composed of a cuff, control module, surface electrodes, and a pressure sensor to detect gait events and trigger stimulation

- Duration of exposure: 30 weeks of MN

- Place of application of intervention: lower limb

Another assistive technology device

- Intervention: control group used AFO. In the first 6 weeks, participants received 8 physical therapy sessions and also received surface sensory stimulation with a TENS device. The physical therapy sessions focused on education on use of the AFO if need, gait training with the AFO, and the development of a home exercise program. Treatment time ranged from 30 to 60 minutes. During the first 3 weeks, participants received surface sensory stimulation on the hemiplegic leg with a TENS device at each physical therapy visit. TENS intensity was set at the lowest stimulation level that yielded a sensory response without motor response, at a frequency of $100 \mathrm{pps}$ and duration of $200 \mu \mathrm{s}$. This stimulation was done for 30 minutes in the first week and for 30 to 45 minutes over the next 2 weeks. After this initial conditioning phase, participants used AFO all day exclusively for ambulation.

- Number of participants: 98

- Device: AFO (articulated, non-articulated, prefabricated or other)

- Duration of exposure: 30 weeks of AFO

- Place of application of intervention: lower limb

Outcomes

Activities involving limbs: walking speed measured with the 10MWT $(\mathrm{m} / \mathrm{s})$

- Outcome type: continuous

- Assessment time point: baseline and 30 weeks

- Device at assessments: baseline and 30-week assessments performed with MN and without MN

Activities involving limbs: fast walking speed measured with the 10MWT $(\mathrm{m} / \mathrm{s})$

- Outcome type: continuous

- Assessment time point: baseline and 30 weeks

- Device at assessments: baseline and 30-week assessments performed with MN and without MN

Activities involving limbs: TUG (s)

- Outcome type: continuous

- Assessment time point: baseline and 30 weeks

- Device at assessments: baseline and 30-week assessments performed with MN and without MN

Exercise capacity: 6MWT (m)

- Outcome type: continuous

- Assessment time point: baseline and 30 weeks

- Device at assessments: baseline and 30-week assessments performed with MN and without MN 
Kluding 2013 (Continued)

Balance: BBS

- Outcome type: continuous

- Assessment time point: baseline and 30 weeks

- Device at assessments: baseline and 30-week assessments performed with MN and without MN

Balance: functional reach $(\mathrm{cm})$

- Outcome type: continuous

- Assessment time point: baseline and 30 weeks

- Device at assessments: baseline and 30-week assessments performed with MN and without MN

Participation scale of HRQoL: SIS - Social participation

- Outcome type: continuous

- Assessment time point: baseline and 30 weeks

Adverse events: dropouts during the intervention period

- Outcome type: binary

Adverse events: serious adverse events related to intervention

- Outcome type: binary

Adverse events: falls

- Outcome type: binary

Author's name: Patricia M Kluding
Institution: Department of Physical Therapy and Rehabilitation Sciences, University of Kansas Medica
Center
Email: pkluding@kumc.edu
Address: University of Kansas Medical Center, 3901 Rainbow Blvd, Mail Stop 3051, Kansas City, KS
66160, USA

Funding source

Bioness Inc

Notes Associated reference: Dunning 2013

\section{Risk of bias}

\begin{tabular}{lll}
\hline Bias & Authors' judgement & Support for judgement \\
\hline $\begin{array}{l}\text { Random sequence genera- } \\
\text { tion (selection bias) }\end{array}$ & Low risk & $\begin{array}{l}\text { Quote: "Once study eligibility was confirmed, random group assignment was } \\
\text { performed by the sponsor using a web-based application prepared by the } \\
\text { study statistician." }\end{array}$ \\
\hline $\begin{array}{l}\text { Allocation concealment } \\
\text { (selection bias) }\end{array}$ & Unclear risk & $\begin{array}{l}\text { Although the study protocol mentioned that the process is concealed by the } \\
\text { site, the method of concealment is not described in sufficient detail to permit a } \\
\text { definitive judgement. }\end{array}$ \\
\hline
\end{tabular}

Blinding of participants High risk There was no blinding of participants and personnel.
and personnel (perfor-
mance bias)
All outcomes

Blinding of outcome assessment (detection bias)
Low risk

Low risk

Quote: "To maintain blinding, a nonblinded research team member coordinates outcome testing and all subjects wear loose pants, a lower leg and shoe

Motor neuroprosthesis for promoting recovery of function after stroke (Review)

Copyright @ 2020 The Cochrane Collaboration. Published by John Wiley \& Sons, Ltd. 
Kluding 2013 (Continued)

All outcomes cover ('gaiter') on the involved lower extremity (to conceal the AFO or FDS cuff and pressure sensor), and an FDS control unit"

Incomplete outcome data Low risk ITT analysis was performed.

(attrition bias)

Secondary outcome

Incomplete outcome data Low risk
(attrition bias)
Primary outcome

\begin{tabular}{lll}
\hline $\begin{array}{l}\text { Selective reporting (re- } \\
\text { porting bias) }\end{array}$ & Low risk & $\begin{array}{l}\text { The study protocol is available, and all of the study's prespecified (primary and } \\
\text { secondary) outcomes that are of interest in the review have been reported in } \\
\text { the prespecified way. }\end{array}$ \\
\hline Other bias & High risk & This trial was funded by Bioness Inc. \\
\hline
\end{tabular}

\section{Kottink 2007}

Study design: RCT
Instruments used: 6MWT, Vicon system, activPAL Professional, surface
ity, SF-36, DIP, EQ-5D
Study design as described in the article: "Randomized controlled trial"
Study duration: not stated
Year of study: not stated

Participants

Inclusion criteria: drop foot identified by an inability to achieve a normal heel strike during walking; first hemiplegia of at least 6 months in duration as a result of a cerebrovascular accident with a stable neurology; individual is an outdoor walker; able to give an informed consent

Exclusion criteria: under age 18 years; passive dorsiflexion of the ankle 5 degrees with knee in extension; medical conditions other than cerebrovascular accident, i.e. neurologic, rheumatic, cardiovascular, or systemic disorders (including diabetes mellitus) limiting the function of walking; injury to deep and superficial peroneal nerve and sciatic nerve; any medical condition that would exclude the use of a surgical procedure or anesthetic; not able to don and doff the equipment; pregnancy

Age: $M N$ group mean age $( \pm S D): 55.2$ years $( \pm 11.36)$; control group mean age $( \pm S D): 52.87$ years $( \pm 9.87)$

Country: the Netherlands

Sample size: 29

Sex: MN group: 10 men and 4 women; control group: 10 men and 5 women

Time poststroke: $\geq 6$ months poststroke. MN group mean time poststroke $( \pm S D)$ : 9.07 years $( \pm 9.29)$; control group mean time poststroke $( \pm S D): 5.67$ years $( \pm 4.64)$

Type of stroke: not stated

Interventions

Motor neuroprosthesis

- Intervention: MN group used STIMUSTEP device. The intervention began with the surgical procedure for the implantation of STIMUSTEP device. After 2 weeks of the surgery the wound was checked and first test stimulation took place. At week 3 the stimulation during walking was tested, and the stimulator was taken home by the participant. In weeks 4 and 5 the use of the stimulator was gradually increased. In weeks 6 to 26 the participants were allowed to use the system all day. 
Kottink 2007 (Continued)

- Number of participants: 14

- Device: a 2-channel electrical stimulator composed of an external transmitter with a built-in antenna, a foot switch, and implantable components consisting of the stimulator, the 2 leads, and the bipolar intraneural electrode. The on-and-off switch of the stimulation was determined by a foot switch sensor. Electrodes are placed under the epineurium of the peroneal nerve.

- Duration of exposure: 26 weeks of MN

- Place of application of intervention: lower limb

Another assistive technology device

- Intervention: the control group continued using their conventional walking device all day for correction of their foot drop (i.e. AFO, orthopedic shoes, or no walking device)

- Number of participants: 15

- Device: polypropylene non-articulated AFO (with 2 crossed posterior steels and an open heel, with a large posterior steel, with a small posterior steel, or with a large posterior steel)

- Duration of exposure: 26 weeks of AFO

- Place of application of intervention: lower limb

Activities involving limbs: walking speed (m/s)
- Outcome type: continuous
- Assessment time point: baseline and 26 weeks
- Device at assessments: baseline assessment performed without MN, 26-week assessment performed
with MN
Participation scale of HRQoL: SF-36 - Social functioning
- Outcome type: continuous
- Assessment time point: baseline and 26 weeks
Adverse events: dropouts during the intervention period
- Outcome type: binary

Identification

Author's name: Anke I Kottink

Institution: Roessingh Research and Development

Email: a.kottink@rrd.nl

Address: Roessingh Research and Development, PO Box 310, 7500 AH, Enschede, the Netherlands

\begin{tabular}{ll}
\hline Funding source & Not reported \\
\hline Notes & This study consisted of 4 articles that were part of a PhD thesis (Kottink 2007; Kottink 2008; Kottink \\
2010; Kottink 2012). \\
We did not include outcomes of 6MWT and walking speed assessed with and without devices because \\
these data were presented only as figures (Kottink 2007; Kottink 2008). We contacted the principal in- \\
vestigator, but the author did not respond to our request for data. \\
References associated with this study: Kottink 2008; Kottink 2010; Kottink 2012
\end{tabular}

\section{Risk of bias}

\begin{tabular}{lll}
\hline Bias & Authors' judgement & Support for judgement \\
\hline $\begin{array}{l}\text { Random sequence genera- } \\
\text { tion (selection bias) }\end{array}$ & Unclear risk & $\begin{array}{l}\text { The study authors stated that blocked randomization was used, but it is not } \\
\text { clear if the method used for selecting the blocks describes a random compo- } \\
\text { nent in the sequence generation process. }\end{array}$ \\
\hline
\end{tabular}


Kottink 2007 (Continued)

$\begin{aligned} & \text { Allocation concealment } \\ & \text { (selection bias) }\end{aligned} \quad$ Low risk Random procedure was carried out by an independent person.

Blinding of participants

High risk

There was no blinding of participants and personnel.

and personnel (perfor-

mance bias)

All outcomes

Blinding of outcome as-
sessment (detection bias) $\quad$ Unclear risk Insufficient information to permit judgement of 'low risk' or 'high risk'

sessment (detection bias)

All outcomes

Incomplete outcome data Low risk ITT analysis was performed.
(attrition bias)

Secondary outcome

Incomplete outcome data High risk

(attrition bias)

Primary outcome
The study had withdrawals, and no ITT was performed for the primary outcome of 10MWT (Kottink 2012).

Selective reporting (re- Unclear risk Insufficient information to permit judgement of 'low risk' or 'high risk'
porting bias)

Other bias Low risk No other bias detected.

Sheffler 2013a

Study design: RCT
Instruments used: lower limb portion of the Fugl-Meyer Assessment, mEFAP, SSQoL, gait analysis with
Vicon system
Study design as described in the article: "Single-blinded randomized controlled trial"
Study duration: not stated
Year of study: not stated

Participants

Inclusion criteria: age $\geq 18$ years, $\geq 12$ weeks poststroke with unilateral hemiparesis and ankle dorsiflexion strength of $\leq 4 / 5$ on the Medical Research Council (MRC) scale. Participants were required to ambulate $\geq 30$ feet without an AFO, score $\geq 24$ on the BBS, and demonstrate correction of foot drop using a PNS without evidence of knee hyperextension during stance.

Exclusion criteria: lower extremity edema, skin breakdown, or absent sensation; serious cardiac arrhythmias, pacemakers or other implanted electronic systems; pregnancy; uncontrolled seizure disorder; concomitant lower motor neuron dysfunction and non-stroke upper motor neuron dysfunction; uncompensated hemineglect; sensory or motor peripheral neuropathy; fixed ankle plantarflex or contracture; or lower extremity botulinum toxin injection within the 3 months prior to study enrollment

Age: $M N$ group mean age $( \pm S D): 52.8$ years $( \pm 12.2)$; control group mean age $( \pm S D): 53.2$ years $( \pm 10.1)$

Country: USA

Sample size: 110 participants

Sex: MN group: 30 men and 24 women; control group: 37 men and 19 women

Time poststroke: > 12 weeks poststroke. MN group mean time poststroke $( \pm S D): 44.7$ months $( \pm 97.5)$; control group mean time poststroke $( \pm \mathrm{SD}): 44.9$ months $( \pm 79.2)$ 
Interventions

\section{Motor neuroprosthesis}

- Intervention: MN group used Odstock Dropped-Foot Stimulator (ODFS) device up to 8 hours per day once device safety was demonstrated. In the first 5 weeks the Functional Training phase $(2 \times 1$-hour sessions per week) took place, in which participants were trained to use the MN device for home and community mobility with an assistive device, such as a straight cane, quad cane, or walker, if needed. Activities included passive and active range-of-motion exercises, lower extremity strengthening, standing balance and weight-shifting activities to the affected limb with transition to least-restrictive assistive device, and refinement of a reciprocal gait pattern. Exercises were done with multiple repetitions with an increase in difficulty and decrease in cues, with and without the MN, as appropriate. In the last 7 weeks the Post-Functional Training phase ( $3 \times 1$-hour sessions) took place, in which device function, application, and usage guidelines were reviewed with each participant to maximize MN compliance.

- Number of participants: 54

- Device: a single-channel surface stimulator with surface electrodes. The stimulation was triggered by an insole pressure sensor.

- Duration of exposure: 12 weeks of independent use of MN

- Follow-up: 12 and 24 weeks' post-treatment

- Place of application of intervention: lower limb

Another assistive technology device

- Intervention: control group consisted of treatment with AFO or no device up to 8 hours per day. In the first 5 weeks the Functional Training phase ( $2 \times 1$-hour sessions per week) took place, in which participants were trained to use the AFO device for home and community mobility with an assistive device, such as a straight cane, quad cane, or walker, if needed. Activities included passive and active rangeof-motion exercises, lower extremity strengthening, standing balance and weight-shifting activities to the affected limb with transition to less restrictive assistive device, and refinement of a reciprocal gait pattern. Exercises were done with multiple repetitions with an increase in difficulty and decrease in cues, with and without the AFO, as appropriate. In the last 7 weeks the Post-Functional Training phase ( $3 \times 1$-hour sessions) took place, in which device function, application, and usage guidelines were reviewed with each participant to maximize AFO compliance.

- Number of participants: 56 (48 participants used AFO as usual care, and 6 participants used no device)

- Device: a custom-molded hinged AFO with plantarflexion block that was fabricated using conventional techniques

- Duration of exposure: 12 weeks of independent use of AFO

- Place of application of intervention: lower limb
Activities involving limbs: mEFAP (s)

- Outcome type: continuous

- Assessment time point: baseline, 12 weeks, 12 weeks post-treatment, and 24 weeks post-treatment

- Device at assessments: baseline, 12 weeks, 12 weeks post-treatment, and 24 weeks post-treatment assessments performed without $\mathrm{MN}$

Activities involving limbs: walking speed $(\mathrm{m} / \mathrm{s})$

- Outcome type: continuous

- Assessment time point: baseline, 12 weeks, 12 weeks post-treatment, and 24 weeks post-treatment

- Device at assessments: baseline, 12 weeks, 12 weeks post-treatment, and 24 weeks post-treatment assessments performed without MN

Participation scale of HRQoL: SSQoL

- Outcome type: continuous

- Assessment time point: baseline, 12 weeks, 12 weeks post-treatment, and 24 weeks post-treatment 
Sheffler 2013a (Continued)

Adverse events: dropouts during the intervention period

- Outcome type: binary

Adverse events: falls

- Outcome type: binary

\begin{tabular}{ll}
\hline Identification & Author's name: Lynne R Sheffler \\
& Institution: Department of Physical Medicine and Rehabilitation, Case Western Reserve University, \\
Cleveland, OH; Cleveland FES Center; Dept of PM\&R, MetroHealth Rehabilitation Institute of Ohio, USA \\
Email: Isheffler@metrohealth.org \\
Address: MetroHealth Medical Center, 4229 Pearl Road, N5-524, Cleveland, OH 44109, USA \\
\hline Funding source & MetroHealth Medical Center \\
\hline Notes & This study consisted of 2 articles (Sheffler 2013a; Sheffler 2015).
\end{tabular}

\section{Risk of bias}

\begin{tabular}{|c|c|c|}
\hline Bias & Authors' judgement & Support for judgement \\
\hline $\begin{array}{l}\text { Random sequence genera- } \\
\text { tion (selection bias) }\end{array}$ & Low risk & $\begin{array}{l}\text { The investigators described that envelopes were used as a random compo- } \\
\text { nent in the sequence generation process. }\end{array}$ \\
\hline $\begin{array}{l}\text { Allocation concealment } \\
\text { (selection bias) }\end{array}$ & Unclear risk & $\begin{array}{l}\text { Although the investigators stated that the randomization sequence was con- } \\
\text { cealed, there is no mention as to whether the envelopes were sealed or not. }\end{array}$ \\
\hline $\begin{array}{l}\text { Blinding of participants } \\
\text { and personnel (perfor- } \\
\text { mance bias) } \\
\text { All outcomes }\end{array}$ & High risk & There was no blinding of participants and personnel. \\
\hline $\begin{array}{l}\text { Blinding of outcome as- } \\
\text { sessment (detection bias) } \\
\text { All outcomes }\end{array}$ & Low risk & Quote: "blinded outcomes assessor". \\
\hline $\begin{array}{l}\text { Incomplete outcome data } \\
\text { (attrition bias) } \\
\text { Secondary outcome }\end{array}$ & Low risk & ITT analysis was performed. \\
\hline $\begin{array}{l}\text { Incomplete outcome data } \\
\text { (attrition bias) } \\
\text { Primary outcome }\end{array}$ & Low risk & ITT analysis was performed. \\
\hline $\begin{array}{l}\text { Selective reporting (re- } \\
\text { porting bias) }\end{array}$ & Low risk & $\begin{array}{l}\text { The study protocol is available, and all of the study's prespecified (primary and } \\
\text { secondary) outcomes that are of interest in the review have been reported in } \\
\text { the prespecified way. }\end{array}$ \\
\hline Other bias & Low risk & No other bias detected. \\
\hline
\end{tabular}

6MWT: 6-minute walk test

10MWT: 10-meter walk test

AFO: ankle-foot orthosis

BDI: Beck Depression Inventory

BBS: Berg Balance Scale 
CABG: coronary artery bypass grafting

DIP: Disability Impact Profile

FAP: Functional Ambulation Profile

FDS: foot drop stimulator

FES: functional electrical stimulation

FRT: Functional Reach Test

HRQoL: health-related quality of life

ITT: intention-to-treat

MMSE: Mini Mental State Exam

mEFAP: modified Emory Functional Ambulation Profile

MI: myocardial infarction

$\mathrm{MN}$ : motor neuroprosthesis

PNS: peroneal nerve stimulation

pps: pulses per second

$\mathrm{RCT}$ : randomized controlled trial

SAE: serious adverse event

SD: standard deviation

SF-36: 36-item Short Form Health Survey

SIS: Stroke Impact Scale

SSQoL: Stroke-Specific Quality of Life

TENS: transcutaneous electrical nerve stimulation

TUG: Timed Up and Go

Characteristics of excluded studies [ordered by study ID]

\begin{tabular}{|c|c|}
\hline Study & Reason for exclusion \\
\hline Alon 2002 & Not randomized \\
\hline Alon 2003a & Not randomized \\
\hline Alon 2007 & Irrelevant intervention \\
\hline Alon 2008 & Irrelevant intervention \\
\hline Baker 2004 & Irrelevant comparison \\
\hline Barrett 2010 & Not randomized \\
\hline Berner 2004 & Not randomized \\
\hline Bundy 2017 & Not randomized \\
\hline Burridge 1997a & Irrelevant intervention \\
\hline Burridge 1997b & Irrelevant outcomes \\
\hline Burridge $1997 c$ & Not randomized \\
\hline Burridge $2007 a$ & Not randomized \\
\hline Burridge 2007b & Not randomized \\
\hline Burridge 2011 & Not randomized \\
\hline Chae 2009 & Irrelevant intervention \\
\hline Chan 2009 & Irrelevant intervention \\
\hline
\end{tabular}

Motor neuroprosthesis for promoting recovery of function after stroke (Review) 


\begin{tabular}{|c|c|}
\hline Study & Reason for exclusion \\
\hline ChiCTR-IOR-17013339 & Irrelevant comparison \\
\hline Daly 2011 & Irrelevant intervention \\
\hline Dujović 2017 & Irrelevant intervention \\
\hline Embrey 2010 & Not eligible cross-over trial \\
\hline Ernst 2013 & Not randomized \\
\hline Everaert 2010 & Not randomized \\
\hline Fujiwara 2009 & Not randomized \\
\hline Gabr 2005 & Not eligible cross-over trial \\
\hline Ghédira 2017 & Irrelevant comparison \\
\hline Granat 1996 & Not randomized \\
\hline Hara 2008 & Irrelevant intervention \\
\hline Hausdorff 2008 & Not randomized \\
\hline Karniel 2019 & Not randomized (quasi-randomized controlled trial) \\
\hline Kim 2016 & Irrelevant comparison \\
\hline Kimberley 2004 & Irrelevant intervention \\
\hline Knutson 2012 & Irrelevant intervention \\
\hline Kojovic 2009 & Irrelevant intervention \\
\hline Laufer 2009 & Not randomized \\
\hline Mann 2011 & Not randomized \\
\hline Martin 2016 & Not randomized \\
\hline Marvulli 2016 & Irrelevant intervention \\
\hline McCabe 2015 & Irrelevant intervention \\
\hline Morone 2012 & Irrelevant intervention \\
\hline NCT03946488 & Not eligible cross-over trial \\
\hline NCT04014270 & Irrelevant intervention (electrical stimulation performed in clinical setting) \\
\hline Ochi 2018 & Irrelevant intervention (electrical stimulation performed in clinical setting) \\
\hline
\end{tabular}




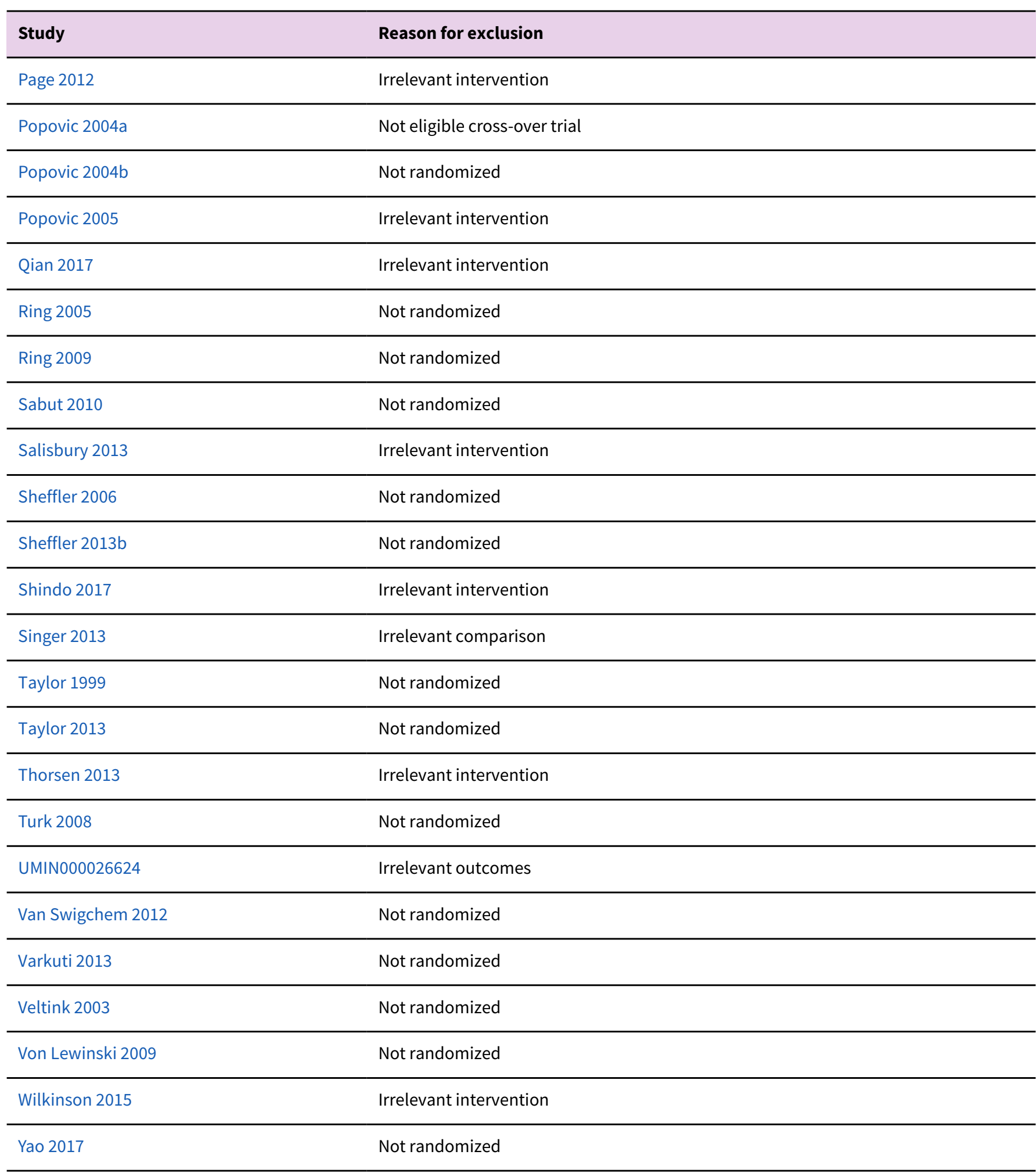

Characteristics of studies awaiting assessment [ordered by study ID]

ISRCTN91639560

Methods Study design: the study author only stated at trial registry that this is a randomized controlled pilot study 
ISRCTN91639560 (Continued)

Instruments used: walking speed; Physiological Cost Index; visual gait analysis from video using Rivermead Visual Gait Assessment; 6MWT; Canadian Occupational Performance Measure; Hospital Anxiety and Depression Scale; Rivermead Mobility Index

Study duration: not stated

Year of study: registered in 2007

Participants

Inclusion criteria: participants will be over 18 years; participants will be medically fit enough to undertake physiotherapy (consultant and GP approval will be sought prior to starting the trial); current inpatient stay will be for rehabilitation following first stroke; during the inpatient period participants will have demonstrated they have sufficient motivation, memory, and cognitive ability to participate in treatment within physiotherapy and practice outside of treatment sessions; participants will be able to understand spoken instructions; participants' goals must include improving gait; suitable participants will be returning home after hospital discharge with a Rivermead Mobility Index of between 6 and 10; participants will be able to attend the hospital for twice-weekly physiotherapy, i.e. will have suitable transport and live within 25 miles of the hospital

Exclusion criteria: unable to tolerate sensation of stimulation (assessed prior to acceptance into the trial); poor skin condition making stimulation unsuitable; previous neurological conditions likely to influence response to treatment; orthopedic/other health problems limiting ability to participate or use stimulation/physiotherapy; score of 25 or under on Mini Mental Test; pacemaker and other active implant users; poorly controlled epileptics; pregnancy

Age: stated only that participants were adults

Sample size: 30 participants

Sex: men and women

Time poststroke: less than 6 months of stroke

Type of stroke: not stated

- Intervention: the experimental group received physiotherapy with the addition of electrical stimulation

- Device: principal investigator only reported that all participants will receive 2 physiotherapy sessions a week for 6 weeks and will also be instructed in exercises to perform at home which include electrical stimulation

- Duration of exposure: 6 weeks

- Place of application of intervention: lower limb

Another assistive technology device

- Intervention: the control group received physiotherapy

- Device: there is no device, only physiotherapy

- Duration of exposure: 6 weeks

- Place of application of intervention: lower limb

- Activities involving limbs: Rivermead Mobility Index

- Exercise capacity: 6MWT 

ment trial

Instruments used: Stroke Upper Limb Capacity Scale (SULCS); Box \& Blocks Test

Study duration: not stated

Year of study: registered in 2018

Participants

Inclusion criteria: 6 to 24 months since a first clinical cortical or subcortical, hemorrhagic or nonhemorrhagic stroke; unilateral upper limb hemiparesis with finger extensor strength of grade no more than 4 out of 5 on the Medical Research Council (MRC) scale; score of at least 1 and no more than 11 out of 14 on the hand section of the upper extremity Fugl-Meyer Assessment; adequate active movement of the shoulder and elbow to position the hand in the workspace for table-top task practice (necessary for the lab task practice sessions); able to follow 3-stage commands; able to recall at least 2 of a list of 3 items after 30 minutes; skin intact on the hemiparetic arm; surface stimulation of the paretic finger and thumb extensors produces functional hand opening without pain (this will exclude those who have too much flexor spasticity); able to hear and respond to cues from stimulator; not receiving occupational therapy (no concomitant occupational therapy); full voluntary opening/closing of the contralateral (less affected) hand; demonstrates ability to follow instructions for operating the stimulator or have a caregiver who will assist them

Exclusion criteria: co-existing neurologic diagnosis of peripheral nerve injury, Parkinson's disease, spinal cord injury, traumatic brain injury, or multiple sclerosis; uncontrolled seizure disorder; brainstem stroke; uncompensated hemineglect; severe shoulder or hand pain; insensate forearm or hand; history of potentially fatal cardiac arrhythmias with hemodynamic instability; implanted electronic systems (e.g. pacemaker); botulinum toxin injections to any upper extremity muscle within 3 months of enrolling; pregnant women due to unknown risks of surface NMES during pregnancy; lack of functional passive range of motion of the wrist or fingers of affected side; diagnosis (apart from stroke) that substantially affects paretic arm and hand function; deficits in communication that interfere with reasonable study participation; lacking sufficient visual acuity to see the stimulator's display; concurrent enrollment in another investigational study

Age: 21 to 90 years

Sample size: 129 participants

Sex: men and women

Time poststroke: not stated

Type of stroke: not stated

- Intervention: the experimental group used a contralaterally controlled FES

- Device: electrical stimulator directed to paretic finger and thumb extensor muscles with the use of surface electrodes. The stimulator will be programmed to deliver stimulation with an intensity that corresponds to the opening of a glove instrumented with sensors and plugged into the stimulator.

- Duration of exposure: 12 weeks

- Place of application of intervention: upper limb

Another assistive technology device

- Intervention: the control group used Cyclic NMES

- Device: electrical stimulator directed to paretic finger and thumb extensor muscles with the use of surface electrodes. The stimulator will be programmed to turn on and off in a repetitive cyclic fashion.

- Duration of exposure: 12 weeks 
NCT03574623 (Continued)

- Place of application of intervention: upper limb
Outcomes

Notes
- Activities involving limbs: Box \& Blocks Test

We contacted the principal investigator to request more detailed information about the intervention to determine if the intervention was used as an orthosis in the home or community context, but as of yet have not received a response.

\section{UMIN000018648}

Study design: the study author only stated at trial registry that this is a randomized cross-over trial
Instruments used: Fugl-Meyer Assessment, Mortor Activity Log, Box \& Blocks Test, Motor Assess-
ment Scale
Study duration: not stated
Year of study: not stated

Participants

Inclusion criteria: time from stroke onset > 5 months; no cognitive deficit; no severe proprioceptive deficit; no severe contracture in paretic hand; independent for locomotion

Exclusion criteria: severe heart failure; severe pulmonary dysfunction; severe hypertension; uncontrolled seizure; pacemaker and other implants; other serious medical condition

Age: 15 to 80 years old

Sample size: 40 participants

Sex: men and women

Time poststroke: not stated

Type of stroke: not stated

- Intervention: the experimental group used HANDS therapy

- Device: HANDS therapy that combines a closed-loop EMG-controlled NMES with a wrist splint

- Duration of exposure: 4 weeks, 8 hours a day

- Place of application of intervention: upper limb

Another assistive technology device

- Intervention: the control group used subthreshold electrical stimulation with HANDS system

- Device: HANDS therapy that combines a closed-loop EMG-controlled NMES with a wrist splint

- Duration of exposure: 4 weeks, 8 hours a day

- Place of application of intervention: upper limb

\section{Outcomes}

- Activities involving limbs: Motor Activity Log

- Activities involving limbs: Box \& Blocks Test sponse. 
Wright 2004

\section{Methods}

Study design: not stated. The study author reported that participants were randomly assigned to groups.

Instruments used: 10MWT, Physiological Cost Index, endurance (3-minute test), modified Ashworth Scale, Rivermead Mobility Index

Study duration: not stated

Year of study: not stated
Inclusion criteria: single stroke of vascular origin with hemiplegia ( $<6$ months); assessed by a clinical specialist physiotherapist to confirm that both a stimulator and an AFO would be suitable for the participant; affected by a drop-foot, identified by failure to achieve a heel strike, and corrected by FES; inability to achieve an effective push-off at terminal stance, identified by clinical observation

Exclusion criteria: use of a dropped-foot stimulator or AFO in the 4 weeks prior to start of the intervention; required an $\mathrm{AFO}$ other than that selected for the trial

Age: not stated

Sample size: 22 participants

Sex: not stated

Time poststroke: not stated

Type of stroke: not stated
Motor neuroprosthesis

- Intervention: experimental group used Odstock Dropped-Foot Stimulator

- Device: a 2-channel surface stimulator with surface electrodes. The stimulation was triggered by an insole pressure sensor.

- Duration of exposure: 24 weeks

- Place of application of intervention: lower limb

Another assistive technology device

- Intervention: the control group used AFO

- Device: Orthomerica Supra-Lite AFO

- Duration of exposure: 24 weeks

- Place of application of intervention: lower limb

- Exercise capacity: the total distance in 3 minutes

6MWT: 6-minute walk test 10MWT: 10-meter walk test AFO: ankle-foot orthosis FES: functional electrical stimulation GP: general practitioner HANDS: Hybrid Assistive Neuromuscular Dynamic Stimulation EMG-controlled NMES: electromyography-controlled neuromuscular electrical stimulation 
Characteristics of ongoing studies [ordered by study ID]

Ghedira 2014

Trial name or title

Randomized controlled trial comparing implanted peroneal nerve stimulation and ankle foot orthosis in spastic paresis

\begin{tabular}{ll}
\hline Methods & Not stated \\
& Random allocation \\
\hline Participants & 24 participants with chronic paresis \\
\hline Interventions & Motor neuroprosthesis \\
- Intervention: implantable motor neuroprosthesis applied to the peroneal nerve during gait and \\
used at home \\
Another assistive technology device \\
- Intervention: ankle-foot orthosis used at home \\
Number of participants: 12
\end{tabular}

\begin{tabular}{ll}
\hline Outcomes & Activities involving limbs: walking speed $(\mathrm{m} / \mathrm{s})$ \\
\hline Starting date & Not stated \\
\hline Contact information & Mouna Ghédira, PhD \\
& Laboratoire ARM - Analyse et Restauration du Mouvement \\
& $\begin{array}{l}\text { Service de Rééducation Neurolocomotrice } \\
\text { CHU Henri Mondor }\end{array}$ \\
& email: mouna.ghedira@aphp.fr \\
\hline Notes & This study was published only as an abstract. We contacted the principal investigator, who report- \\
& ed that the full text has not yet been published. \\
\hline
\end{tabular}

DATA AND ANALYSES

\section{Comparison 1. Motor neuroprosthesis versus another assistive technology device}

\begin{tabular}{lllll}
\hline Outcome or subgroup title & No. of studies & $\begin{array}{l}\text { No. of partici- } \\
\text { pants }\end{array}$ & Statistical method & Effect size \\
\hline $\begin{array}{l}\text { 1 Activities involving limbs: walking } \\
\text { speed until } 6 \text { months of device use }\end{array}$ & 2 & 605 & $\begin{array}{l}\text { Mean Difference (IV, Random, } \\
95 \% \mathrm{Cl})\end{array}$ & $-0.05[-0.10,-0.00]$ \\
\hline $\begin{array}{l}\text { 2 Activities involving limbs: walking } \\
\text { speed between } 6 \text { and } 12 \text { months of } \\
\text { device use }\end{array}$ & 3 & 713 & $\begin{array}{l}\text { Mean Difference (IV, Random, } \\
95 \% \text { Cl) }\end{array}$ & $0.00[-0.05,0.05]$ \\
\hline $\begin{array}{l}\text { 3 Activities involving limbs: walking } \\
\text { speed }\end{array}$ & 4 & 823 & $\begin{array}{l}\text { Mean Difference (IV, Random, } \\
95 \% \text { Cl) }\end{array}$ & $-0.01[-0.06,0.04]$ \\
\hline
\end{tabular}




\begin{tabular}{|c|c|c|c|c|}
\hline Outcome or subgroup title & No. of studies & $\begin{array}{l}\text { No. of partici- } \\
\text { pants }\end{array}$ & Statistical method & Effect size \\
\hline 3.1 Surface MN & 3 & 802 & $\begin{array}{l}\text { Mean Difference (IV, Random, } \\
95 \% \mathrm{CI} \text { ) }\end{array}$ & $-0.02[-0.06,0.02]$ \\
\hline 3.2 Implantable MN & 1 & 21 & $\begin{array}{l}\text { Mean Difference (IV, Random, } \\
95 \% \mathrm{CI})\end{array}$ & $0.12[-0.04,0.28]$ \\
\hline 4 Activities involving limbs: TUG & 2 & 692 & $\begin{array}{l}\text { Mean Difference (IV, Random, } \\
95 \% \mathrm{CI})\end{array}$ & $0.51[-4.41,5.43]$ \\
\hline 5 Activities involving limbs: mEFAP & 2 & 605 & $\begin{array}{l}\text { Mean Difference (IV, Random, } \\
95 \% \mathrm{CI})\end{array}$ & $\begin{array}{l}14.77[-12.52, \\
42.06]\end{array}$ \\
\hline 6 Participation scale of HRQoL & 3 & 632 & $\begin{array}{l}\text { Std. Mean Difference (IV, Ran- } \\
\text { dom, } 95 \% \mathrm{CI} \text { ) }\end{array}$ & $0.26[-0.22,0.74]$ \\
\hline 7 Exercise capacity: 6MWT & 2 & 692 & $\begin{array}{l}\text { Mean Difference (IV, Random, } \\
95 \% \mathrm{CI} \text { ) }\end{array}$ & $-9.03[-26.87,8.81]$ \\
\hline 8 Balance: BBS & 2 & 692 & $\begin{array}{l}\text { Mean Difference (IV, Random, } \\
95 \% \mathrm{CI} \text { ) }\end{array}$ & $-0.34[-1.96,1.28]$ \\
\hline $\begin{array}{l}9 \text { Adverse events: number of } \\
\text { dropouts during the intervention pe- } \\
\text { riod }\end{array}$ & 4 & 829 & $\begin{array}{l}\text { Risk Ratio (M-H, Random, } \\
95 \% \mathrm{Cl})\end{array}$ & $1.48[1.11,1.97]$ \\
\hline $\begin{array}{l}10 \text { Adverse events: serious adverse } \\
\text { events related to intervention/during } \\
\text { the intervention period }\end{array}$ & 2 & 692 & $\begin{array}{l}\text { Risk Ratio (M-H, Random, } \\
95 \% \mathrm{Cl})\end{array}$ & $0.35[0.04,3.33]$ \\
\hline 11 Adverse events: falls & 3 & 802 & $\begin{array}{l}\text { Risk Ratio (M-H, Random, } \\
95 \% \mathrm{Cl} \text { ) }\end{array}$ & $1.20[0.92,1.55]$ \\
\hline
\end{tabular}

Analysis 1.1. Comparison 1 Motor neuroprosthesis versus another assistive technology device, Outcome 1 Activities involving limbs: walking speed until 6 months of device use.

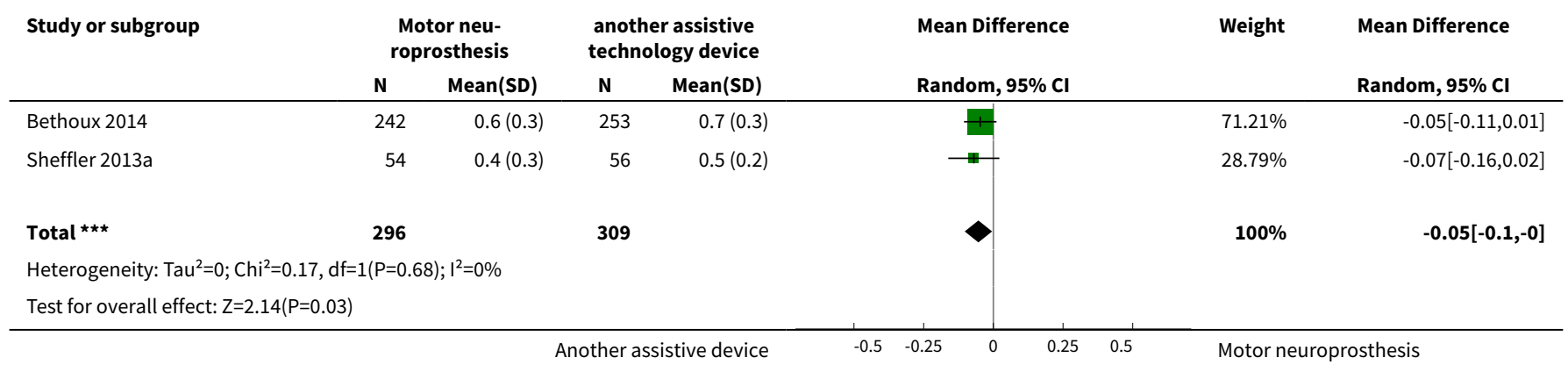


Analysis 1.2. Comparison 1 Motor neuroprosthesis versus another assistive technology device, Outcome 2 Activities involving limbs: walking speed between $\mathbf{6}$ and 12 months of device use.

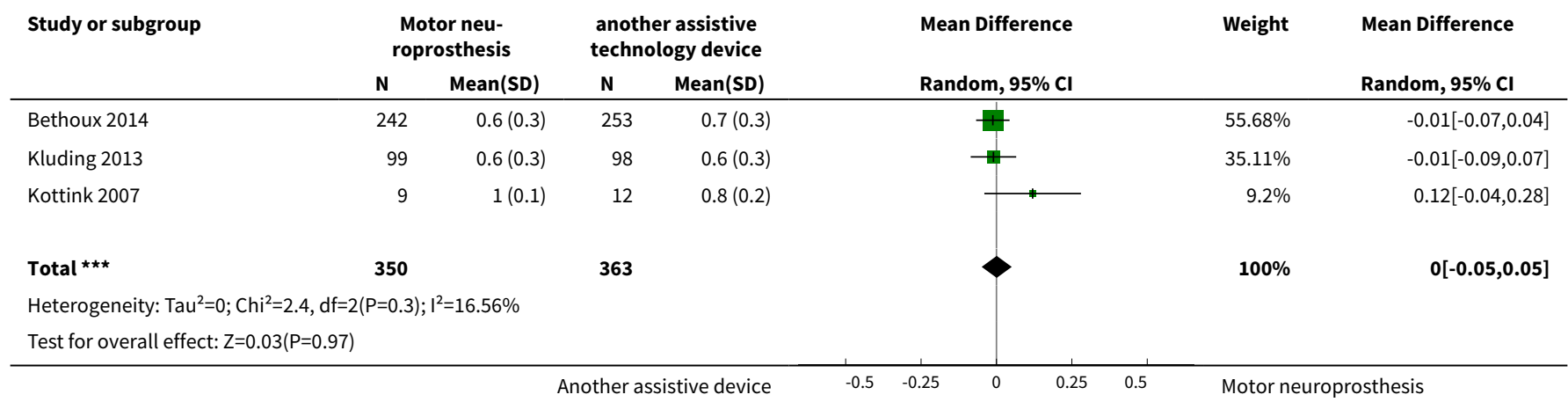

Analysis 1.3. Comparison 1 Motor neuroprosthesis versus another assistive technology device, Outcome 3 Activities involving limbs: walking speed.

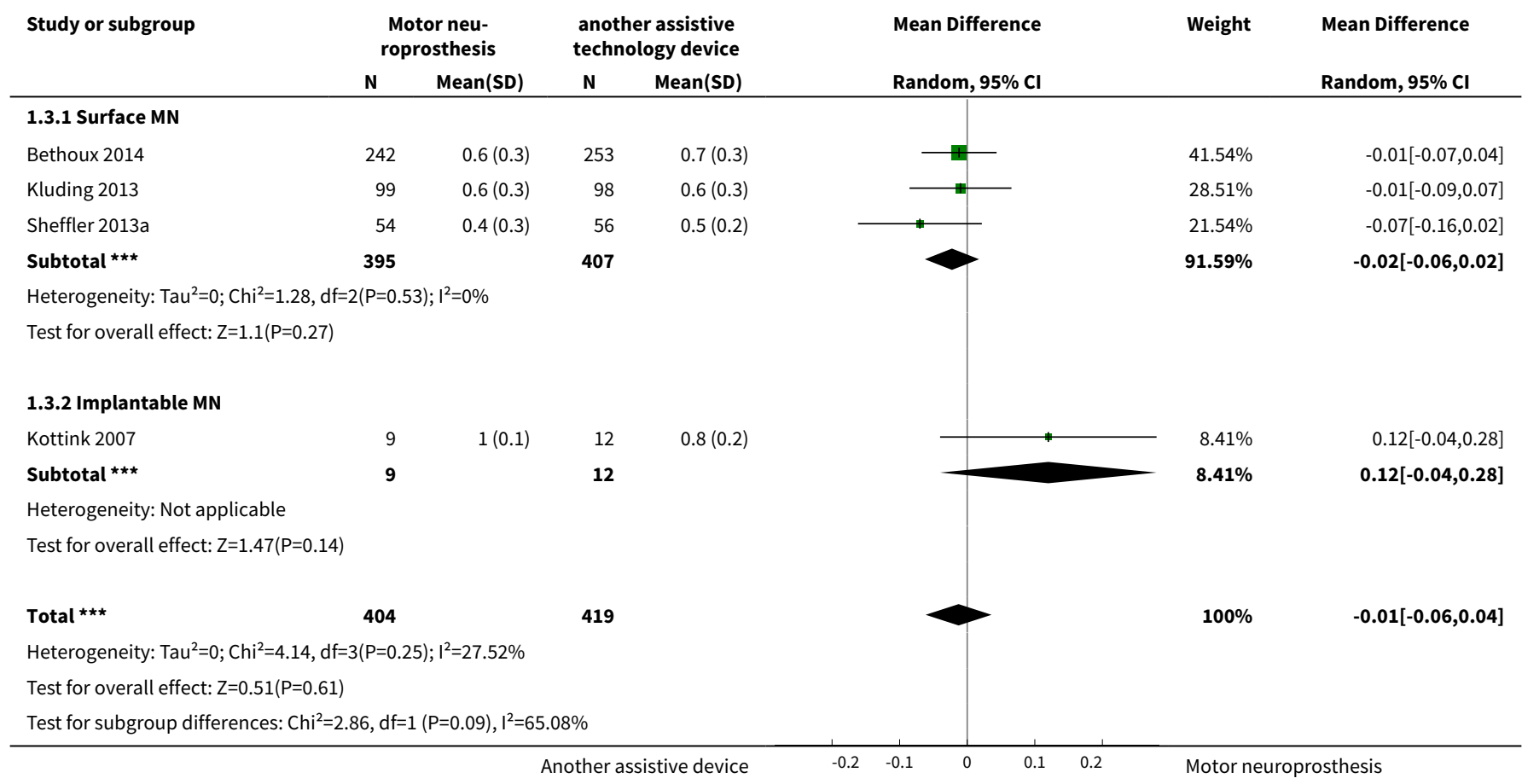

Analysis 1.4. Comparison 1 Motor neuroprosthesis versus another assistive technology device, Outcome 4 Activities involving limbs: TUG.

\begin{tabular}{|c|c|c|c|c|c|c|c|}
\hline \multirow[t]{2}{*}{ Study or subgroup } & \multicolumn{2}{|c|}{$\begin{array}{l}\text { Motor neu- } \\
\text { roprosthesis }\end{array}$} & \multicolumn{2}{|c|}{$\begin{array}{l}\text { another assistive } \\
\text { technology device }\end{array}$} & \multirow{2}{*}{$\begin{array}{l}\text { Mean Difference } \\
\text { Random, } 95 \% \mathrm{Cl}\end{array}$} & \multirow[t]{2}{*}{ Weight } & \multirow{2}{*}{$\begin{array}{l}\text { Mean Difference } \\
\text { Random, } 95 \% \mathrm{Cl}\end{array}$} \\
\hline & $\mathbf{N}$ & Mean(SD) & $\mathbf{N}$ & Mean(SD) & & & \\
\hline Kluding 2013 & 99 & $29(24)$ & 98 & $28.1(27.8)$ & -1 & $46.14 \%$ & $0.88[-6.36,8.12]$ \\
\hline Bethoux 2014 & 242 & $27.2(42)$ & 253 & $27(33.4)$ & . & $53.86 \%$ & $0.2[-6.5,6.9]$ \\
\hline
\end{tabular}




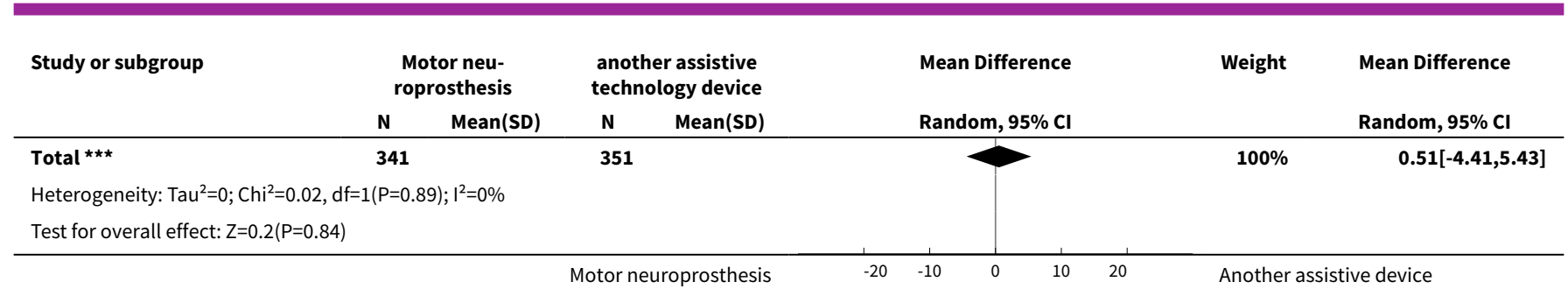

Analysis 1.5. Comparison 1 Motor neuroprosthesis versus another assistive technology device, Outcome 5 Activities involving limbs: mEFAP.

\begin{tabular}{|c|c|c|c|c|c|c|c|}
\hline \multirow[t]{2}{*}{ Study or subgroup } & \multicolumn{2}{|c|}{$\begin{array}{l}\text { Motor neu- } \\
\text { roprosthesis }\end{array}$} & \multicolumn{2}{|c|}{$\begin{array}{l}\text { another assistive } \\
\text { technology device }\end{array}$} & \multirow{2}{*}{$\begin{array}{l}\text { Mean Difference } \\
\text { Random, } 95 \% \mathrm{CI}\end{array}$} & \multirow[t]{2}{*}{ Weight } & \multirow{2}{*}{$\begin{array}{l}\text { Mean Difference } \\
\text { Random, } 95 \% \mathrm{Cl}\end{array}$} \\
\hline & $\mathbf{N}$ & $\operatorname{Mean}(S D)$ & $\mathbf{N}$ & Mean(SD) & & & \\
\hline Bethoux 2014 & 242 & $498(497.8)$ & 253 & $\begin{array}{r}479.4 \\
(609.2)\end{array}$ & - & $7.78 \%$ & $18.6[-79.22,116.42]$ \\
\hline Sheffler 2013a & 54 & $107.9(78.8)$ & 56 & $93.5(73)$ & & $92.22 \%$ & $14.45[-13.97,42.87]$ \\
\hline 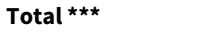 & 296 & & 309 & & & $100 \%$ & $14.77[-12.52,42.06]$ \\
\hline \multicolumn{8}{|c|}{ Heterogeneity: $\operatorname{Tau}^{2}=0 ; \mathrm{Chi}^{2}=0.01, \mathrm{df}=1(\mathrm{P}=0.94) ;\left.\right|^{2}=0 \%$} \\
\hline \multicolumn{3}{|c|}{ Test for overall effect: $Z=1.06(P=0.29)$} & & & & & \\
\hline
\end{tabular}

Analysis 1.6. Comparison 1 Motor neuroprosthesis versus another assistive technology device, Outcome 6 Participation scale of HRQoL.

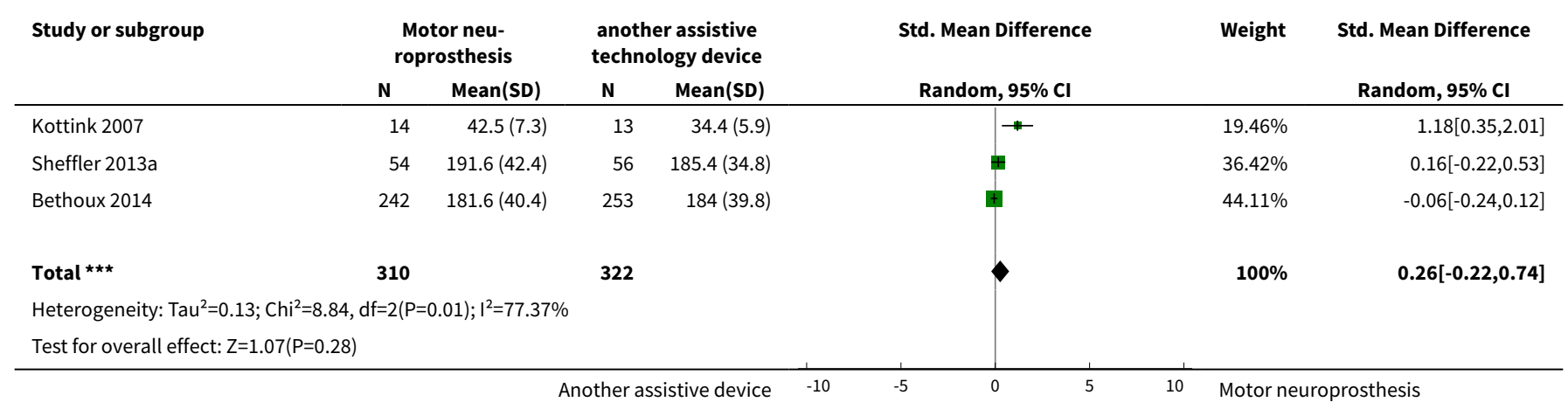

Analysis 1.7. Comparison 1 Motor neuroprosthesis versus another assistive technology device, Outcome 7 Exercise capacity: 6MWT.

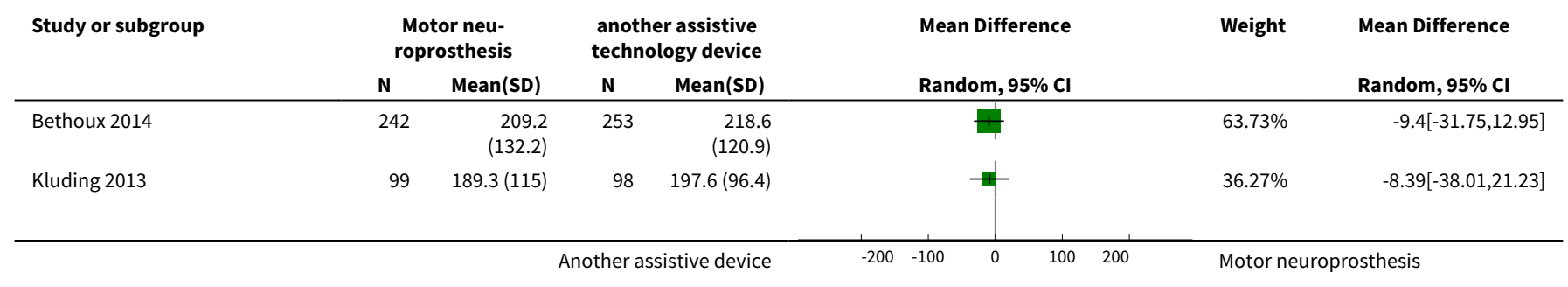




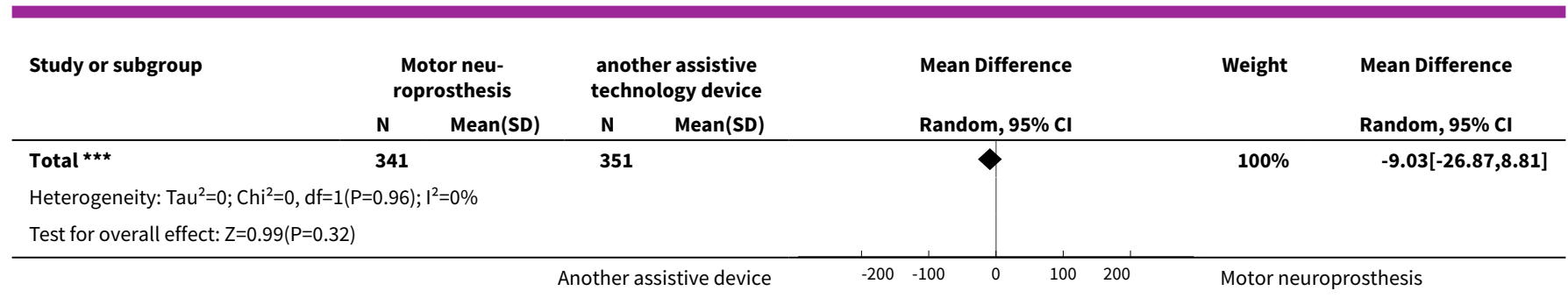

Analysis 1.8. Comparison 1 Motor neuroprosthesis versus another assistive technology device, Outcome 8 Balance: BBS.

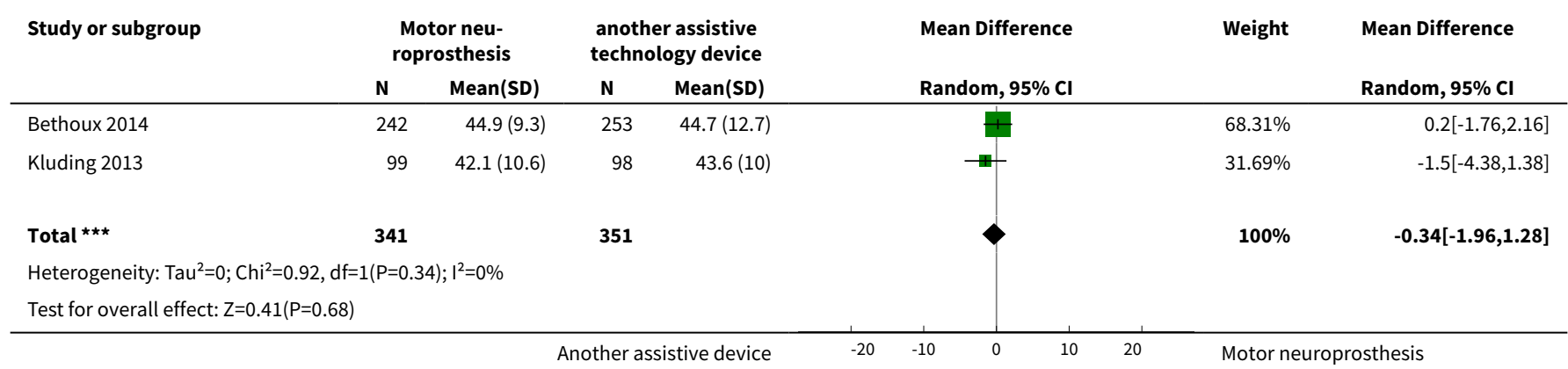

Analysis 1.9. Comparison 1 Motor neuroprosthesis versus another assistive technology device, Outcome 9 Adverse events: number of dropouts during the intervention period.

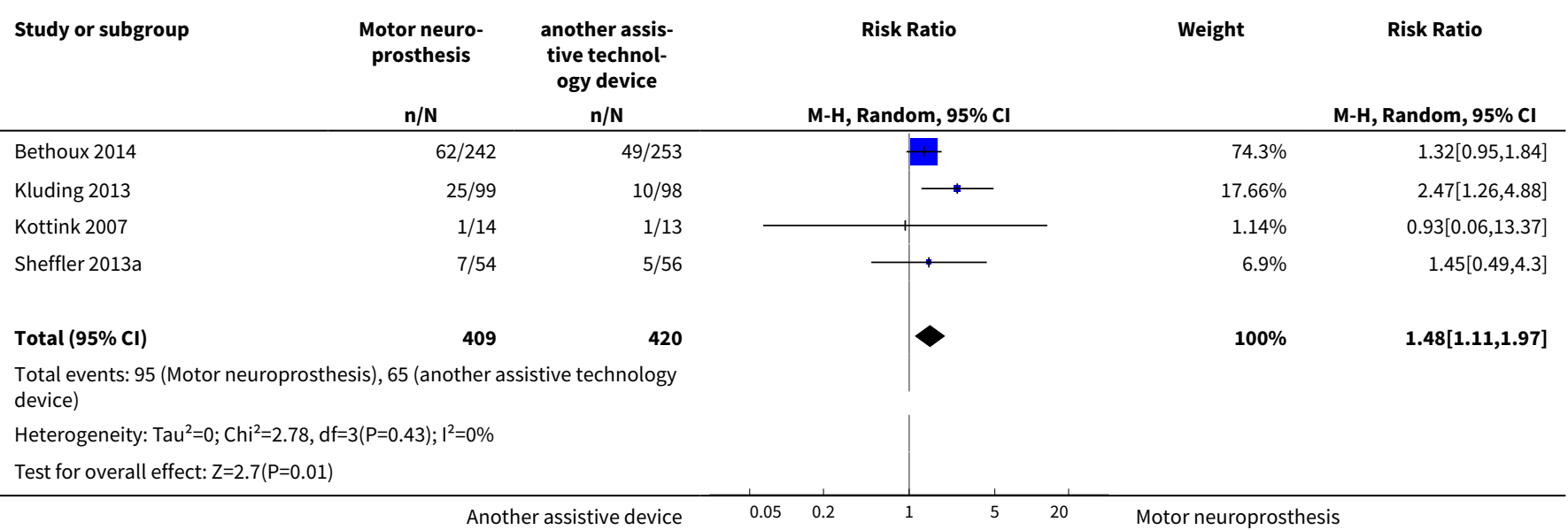


Analysis 1.10. Comparison 1 Motor neuroprosthesis versus another assistive technology device, Outcome 10 Adverse events: serious adverse events related to intervention/during the intervention period.

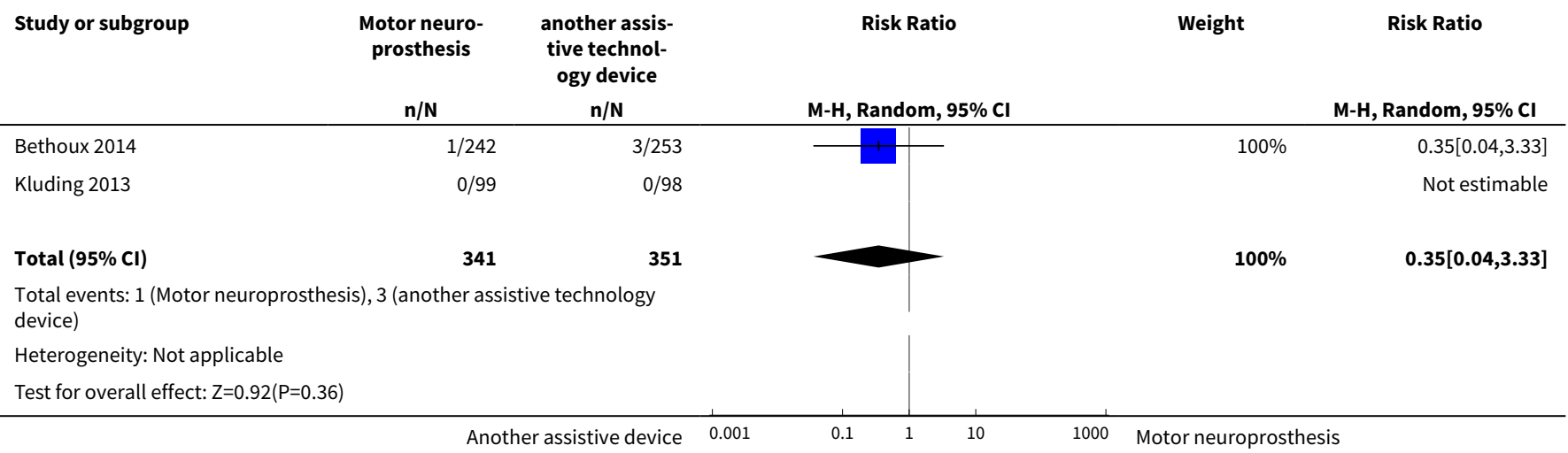

Analysis 1.11. Comparison 1 Motor neuroprosthesis versus another assistive technology device, Outcome 11 Adverse events: falls.

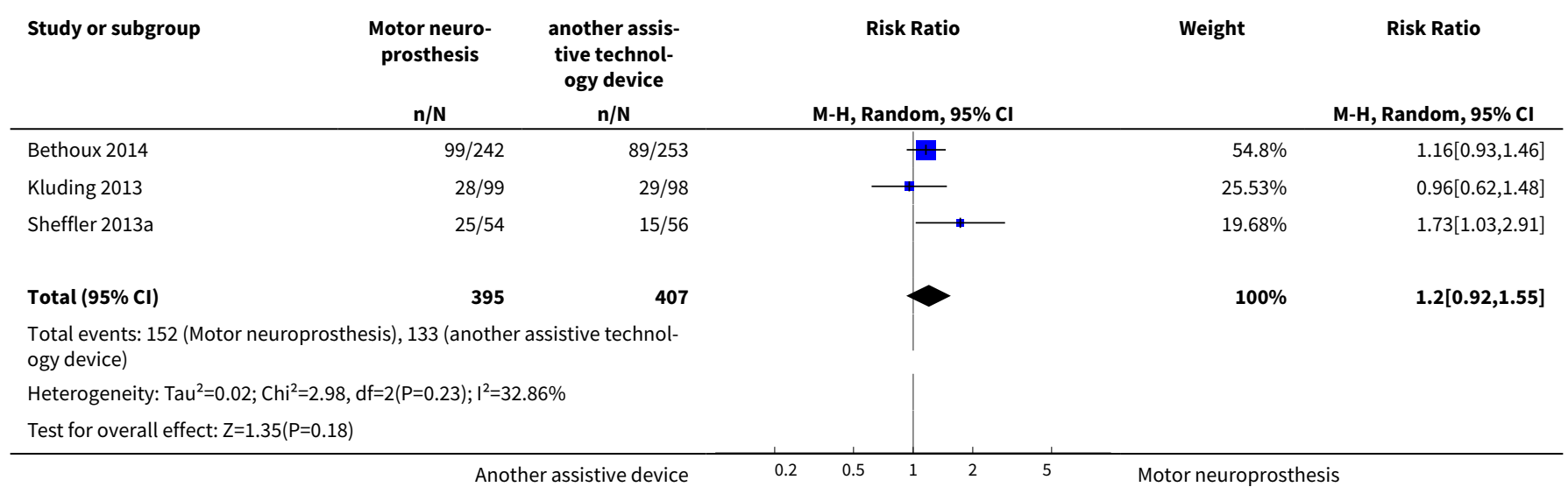

\section{ADDITIONAL TABLES}

Table 1. Intervention characteristics of the $\mathrm{MN}$ used in the included trials

\begin{tabular}{|c|c|c|c|c|}
\hline $\begin{array}{l}\text { Study ID (re- } \\
\text { port) }\end{array}$ & MN device & $\begin{array}{l}\text { Duration of ex- } \\
\text { posure to MN in- } \\
\text { tervention }\end{array}$ & $\begin{array}{l}\text { Conditioning protocol used to } \\
\text { adapt participants to } \mathrm{MN} \text { use }\end{array}$ & $\begin{array}{l}\text { MN use/daily } \\
\text { use for increas- } \\
\text { ing the activi- } \\
\text { ties and partic- } \\
\text { ipation in the } \\
\text { home or com- } \\
\text { munity context }\end{array}$ \\
\hline Bethoux 2014 & $\begin{array}{l}\text { The MN used was the WalkAide } \\
\text { device (Innovative Neurotronics, } \\
\text { Austin, TX, USA). It is a commer- } \\
\text { cially available, battery-operat- } \\
\text { ed, single-channel surface per- } \\
\text { oneal nerve stimulator that con- } \\
\text { sists of a cuff worn around the }\end{array}$ & $\begin{array}{l}\text { The duration of } \\
\text { MN intervention } \\
\text { was } 12 \text { months. } \\
\text { The condition- } \\
\text { ing protocol oc- } \\
\text { curred in the first } \\
2 \text { weeks, after }\end{array}$ & $\begin{array}{l}\text { The first part consisted of fitting } \\
\text { and programming the MN device } \\
\text { as well as patient education per- } \\
\text { formed by WalkAide-certified or- } \\
\text { thotist or licensed physical thera- } \\
\text { pist. The conditioning protocol in- }\end{array}$ & $\begin{array}{l}\text { Participants } \\
\text { were instruct- } \\
\text { ed to wear MN } \\
\text { device on a } \\
\text { full-time basis } \\
\text { (quote: "ie, for all } \\
\text { walking activities }\end{array}$ \\
\hline
\end{tabular}


Table 1. Intervention characteristics of the $\mathrm{MN}$ used in the included trials (Continued)

proximal part of the lower leg, which holds the control module and surface electrodes. This device uses a tilt sensor and an accelerometer to trigger ankle dorsiflexion and control the timing and duration of peroneal nerve stimulation during the swing phase of gait to alleviate foot drop.

Kluding $2013 \quad$ The MN used was the NESS L300 device (Bioness Inc, Valencia, CA, USA). It is a commercially available, battery-operated, single-channel surface peroneal nerve stimulator that consists of a cuff with integrated stimulation unit and electrodes, a control unit, and an in-shoe pressure sensor. The pressure sensor detects heel off and initial contact events during gait. It transmits wireless signals to the stimulation cuff, which initiates or pauses the stimulation of deep and superficial branches of the peroneal nerve via 2 surface electrodes that activate dorsiflexors and evertors muscles to ensure foot clearance during the swing phase of gait and prevent excessive ankle inversion during early stance. which participants started daily use of MN device. cluded a 2-week progressive wearing schedule of MN device.

throughout the day").

\section{The duration of $\mathrm{MN}$ intervention was 30 weeks. The condition- ing protocol oc- curred in the first 6 weeks. Partic- ipants used the MN device all day between week 4 and week 30}

The first part consisted of initial fitting of the device, gait training, wearing schedule, home exercise program, and participant education based on manufacturer standardized protocols. For the first 3 weeks, participants followed the standard conditioning protocol (gradually increasing walking with the MN from 15 minutes each day to all-day use). During the same period, participants also used the MN for cyclic stimulation while not walking in order to gradually strengthen and condition the muscles to avoid fatigue when using the device (Dunning 2013).. During the first 6 weeks of the study, participants also received 8 dosematched sessions of physical therapy. The first 2 to 4 therapy visits focused on education on device use, initial gait training, and an individualized home exercise program. The remaining physical therapy sessions focused on gait training (Kluding 2013).
Kottink 2007

\author{
The MN used was the STIMUSTEP \\ device (FineTech Medical Ltd, \\ Hertfordshire, UK). It is a com- \\ mercially available, battery-op- \\ erated, 2-channel implantable \\ device composed of implantable \\ components such as a stimula- \\ tor, 2 leads, and bipolar intra- \\ neural electrodes, and non-im- \\ plantable components such as \\ an external transmitter with a \\ built-in antenna and a pressure \\ sensor. 1 electrode is surgically \\ positioned under the epineuri- \\ um of the superficial peroneal \\ nerve and the other under the \\ epineurium of the deep peroneal \\ nerve. This device promotes the \\ ankle dorsiflexion/eversion dur- \\ ing gait to correct foot drop, and \\ a pressure sensor placed inside
}
The duration of MN inter- vention was 26 weeks. The in- tervention be- gan with the sur- gical procedure for placement of the implant. After 2 weeks of the surgery, the wound was
Quote: "Two weeks after the surgery the wound was checked and a first test stimulation took place. In the third week, stimula- tion during walking was tested and the stimulator was taken home by the patient. The use of the stimu- lator was gradually increased over 2 weeks to prevent severe muscle pain and fatigue. After this period patients were allowed to use the system all day." checked and first test stimulation took place. The conditioning protocol began at the third week, and allday MN use be-
Participants used the $\mathrm{MN}$ all day for ambulation (Dunning 2013).*
Participants were allowed to use the system all day between week 6 and week 26. 
Table 1. Intervention characteristics of the $\mathrm{MN}$ used in the included trials (Continued)

the shoe determines the on and off switching of the stimulation.

\begin{abstract}
The MN used to correct foot drop was the Odstock Dropped-Foot Stimulator (ODFS) device (Odstock Medical Ltd, Salisbury Wiltshire, UK). The ODFS is a commercially available, battery-operated, single-channel surface peroneal nerve stimulator consisting of an electrical stimulator, a control module, pressure sensors, and surface electrodes. The stimulation is triggered by an insole pressure-sensing foot switch that detects heel rise at pre-swing.
\end{abstract}

gan at the sixth

week.

The duration of $\mathrm{MN}$ intervention was 12 weeks. The conditioning protocol occurred over the 12 weeks. Daily $\mathrm{MN}$ use began once device safety was demonstrated by participants.
In the first 5 weeks the Functional Training phase $(2 \times 1$-hour sessions per week) took place, in which participants were trained to use MN device for home and community mobility with an assistive device, if needed. Activities included passive and active range-ofmotion exercises, lower extremity strengthening, standing balance and weight-shifting activities to the affected limb with transition to least-restrictive assistive device, and refinement of a reciprocal gait pattern. Exercises were done with multiple repetitions with an increase in difficulty and a decrease in cues, with and without the MN device, as appropriate. In the last 7 weeks the Post-Functional Training Phase ( $3 \times 1$-hour sessions) took place, in which device function, application, and usage guidelines were reviewed with each participant to maximize MN compliance.
The article did not explicitly mention when participants started all-day MN use, but reported that as soon as participants demonstrated safe use of the device, it was used up to 8 hours per day.

MN: motor neuroprosthesis

*Dunning 2013 corresponds to the published protocol of the study Kluding 2013.

Table 2. Outcome measures used from the included trials

\begin{tabular}{|c|c|c|c|c|c|}
\hline Study ID (report) & $\begin{array}{l}\text { Indepen- } \\
\text { dence in ADL }\end{array}$ & Activities involving limbs & $\begin{array}{l}\text { Participation } \\
\text { scales of HRQOL }\end{array}$ & $\begin{array}{l}\text { Exercise ca- } \\
\text { pacity }\end{array}$ & Balance \\
\hline $\begin{array}{l}\text { Bethoux } 2014 \\
\text { (Bethoux 2014; 6-month } \\
\text { assessment) }\end{array}$ & - & $\begin{array}{l}\text { Comfortable walking speed } \\
\text { measured by } 10 M W T \text {, TUG, } \\
\text { mEFAP }\end{array}$ & $\begin{array}{l}\text { SSQoL (total val- } \\
\text { ue); SIS (all do- } \\
\text { mains) }\end{array}$ & 6MWT & BBS \\
\hline $\begin{array}{l}\text { Bethoux } 2014 \\
\text { (Bethoux 2015; 12-month } \\
\text { assessment) }\end{array}$ & - & $\begin{array}{l}\text { Comfortable walking speed } \\
\text { measured by } 10 \mathrm{MWT}, \text { mEFAP }\end{array}$ & - & 6MWT & - \\
\hline $\begin{array}{l}\text { Kluding } 2013 \text { (Kluding } \\
\text { 2013) }\end{array}$ & - & $\begin{array}{l}\text { Comfortable and fast walking } \\
\text { speed measured by 10MWT, } \\
\text { TUG }\end{array}$ & $\begin{array}{l}\text { SIS (ADL/iADL, Mo- } \\
\text { bility, Participa- } \\
\text { tion domains) }\end{array}$ & 6MWT & BBS; FRT \\
\hline $\begin{array}{l}\text { Kottink } 2007 \text { (Kottink } \\
\text { 2007; Kottink 2008; Kot- } \\
\text { tink 2010; Kottink 2012) }\end{array}$ & - & $\begin{array}{l}\text { Comfortable walking speed } \\
\text { motion analysis system }\end{array}$ & $\begin{array}{l}\text { SF-36 (all do- } \\
\text { mains) }\end{array}$ & - & - \\
\hline
\end{tabular}


Table 2. Outcome measures used from the included trials (Continued)

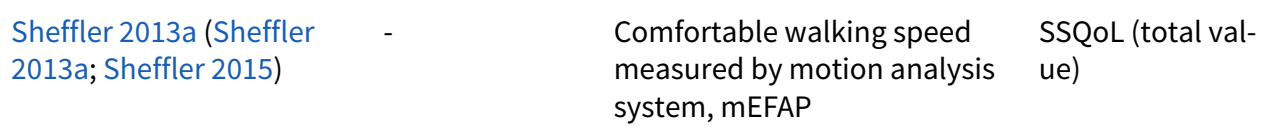

6MWT: 6-minute walk test 10MWT: 10-meter walk test ADL: activities of daily living BBS: Berg Balance Scale FRT: Functional Reach Test HRQoL: health-related quality of life iADL: instrumental activities of daily living mEFAP: modified Emory Functional Ambulation Profile SF-36: 36-item Short Form Health Survey SIS: Stroke Impact Scale SSQoL: Stroke-Specific Quality of Life TUG: Timed Up and Go test

Table 3. Dropouts

\begin{tabular}{|c|c|c|}
\hline Study ID (report) & Motor neuroprosthesis & Another assistive technology device \\
\hline $\begin{array}{l}\text { Bethoux } 2014 \\
\text { (Bethoux 2014; 6-month } \\
\text { assessment)* }\end{array}$ & $\begin{array}{l}2 \text { deceased; } 25 \text { non-compliance with protocol; } 15 \\
\text { participant request; } 7 \text { medical reasons; } 4 \text { lost to } \\
\text { follow-up; } 2 \text { investigator withdrew }\end{array}$ & $\begin{array}{l}2 \text { deceased; } 13 \text { non-compliance with protocol; } 18 \\
\text { participant request; } 4 \text { medical reasons; } 3 \text { lost to fol- } \\
\text { low-up; } 1 \text { investigator withdrew }\end{array}$ \\
\hline $\begin{array}{l}\text { Bethoux } 2014 \\
\text { (Bethoux 2015; 12- } \\
\text { month assessment) }\end{array}$ & $\begin{array}{l}2 \text { deceased; } 25 \text { non-compliance with protocol; } 16 \\
\text { participant request; } 7 \text { medical reasons; } 6 \text { lost to } \\
\text { follow-up; } 6 \text { investigator withdrew }\end{array}$ & $\begin{array}{l}3 \text { deceased; } 15 \text { non-compliance with protocol; } 19 \\
\text { participant request; } 4 \text { medical reasons; } 6 \text { lost to fol- } \\
\text { low-up; } 2 \text { investigators withdrew }\end{array}$ \\
\hline $\begin{array}{l}\text { Kluding } 2013 \text { (Kluding } \\
\text { 2013) }\end{array}$ & 2 lost to follow-up; 23 discontinued intervention & 1 lost to follow-up; 9 discontinued intervention \\
\hline $\begin{array}{l}\text { Kottink } 2007 \text { (Kottink } \\
\text { 2007; Kottink 2008; Kot- } \\
\text { tink 2010; Kottink 2012) }\end{array}$ & 1 technical defect in the epineural electrode & 1 psychological issues not related to the study \\
\hline $\begin{array}{l}\text { Sheffler 2013a (Sheffler } \\
\text { 2013a) }\end{array}$ & $\begin{array}{l}6 \text { non-medical reasons; } 1 \text { medical reason } \\
\text { - } 12 \text {-week follow-up: } 2 \text { non-medical reasons } \\
\text { - } 24 \text {-week follow-up: } 4 \text { non-medical reasons, } 1 \\
\text { medical reason }\end{array}$ & $\begin{array}{l}2 \text { non-medical reasons; } 3 \text { medical reasons } \\
\text { - } 12 \text {-week follow-up: } 1 \text { non-medical reason } \\
\text { - 24-week follow-up: } 3 \text { non-medical reasons, } 1 \text { med- } \\
\text { ical reason }\end{array}$ \\
\hline
\end{tabular}

*Bethoux 2014 (six-month assessment) corresponds to the first report of Bethoux 2014 study whose assessment was made after six months of motor neuroprosthesis use.

**Bethoux 2014 (12-month assessment) corresponds to the second report of Bethoux 2014 study whose assessment was made after 12 months of motor neuroprosthesis use.

Table 4. Sensitivity analysis excluding studies from the analysis that were at high risk of bias for blinding of outcome assessors

\begin{tabular}{lll}
\hline Outcome & Study ID (report) & Analysis results \\
\hline $\begin{array}{l}\text { Activities involving limbs: walking } \\
\text { speed until } 6 \text { months of device use }\end{array}$ & Sheffler 2013a & $\mathrm{MD}-0.07,95 \% \mathrm{Cl}-0.16$ to $0.02 ; \mathrm{P}=0.13 ;$ participants $=110 ; 12=0 \%$ \\
\hline
\end{tabular}


Table 4. Sensitivity analysis excluding studies from the analysis that were at high risk of bias for blinding of outcome assessors (Continued)

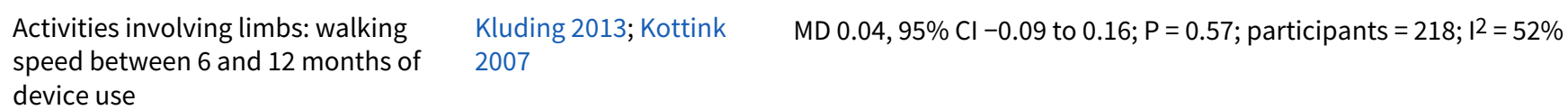

\begin{tabular}{|c|c|c|}
\hline Activities involving limbs: TUG & Kluding 2013 & MD $0.88,95 \% \mathrm{Cl}-6.36$ to $8.12 ; \mathrm{P}=0.81 ;$ participants $=197 ;\left.\right|^{2}=0 \%$ \\
\hline Activities involving limbs: mEFAP & Sheffler 2013a & $\begin{array}{l}\text { MD } 14.45,95 \% \mathrm{Cl}-13.97 \text { to } 42.87 ; \mathrm{P}=0.32 ; \text { participants }=110 ; \mathrm{I}^{2}= \\
0 \%\end{array}$ \\
\hline Participation scale of HRQoL & $\begin{array}{l}\text { Kottink 2007; Sheffler } \\
\text { 2013a }\end{array}$ & $\begin{array}{l}\text { SMD } 0.60,95 \% \mathrm{Cl}-0.39 \text { to } 1.59 ; \mathrm{P}=0.24 ; \text { participants }=137 ;\left.\right|^{2}= \\
79 \%\end{array}$ \\
\hline Exercise capacity: 6MWT & Kluding 2013 & $\begin{array}{l}\mathrm{MD}-8.39,95 \% \mathrm{Cl}-38.01 \text { to } 21.23 ; \mathrm{P}=0.58 ; \text { participants }=197 ; \mathrm{I}^{2}= \\
0 \%\end{array}$ \\
\hline Balance: BBS & Kluding 2013 & $\mathrm{MD}-1.50,95 \% \mathrm{Cl}-4.38$ to $1.38 ; \mathrm{P}=0.31 ;$ participants $=197 ;\left.\right|^{2}=0 \%$ \\
\hline
\end{tabular}

6MWT: 6-minute walk test

BBS: Berg Balance Scale

$\mathrm{Cl}$ : confidence interval

HRQoL: health-related quality of life

MD: mean difference

mEFAP: modified Emory Functional Ambulation Profile

SMD: standardized mean difference

TUG: Timed Up and Go test

\section{A P PE N DICES}

\section{Appendix 1. The Cochrane Central Register of Controlled Trials (CENTRAL) search strategy}

\begin{tabular}{ll}
\hline$\# 1$ & MeSH descriptor: [Cerebrovascular Disorders] explode all trees \\
\hline$\# 2$ & MeSH descriptor: [Basal Ganglia Cerebrovascular Disease] explode all trees \\
\hline$\# 3$ & MeSH descriptor: [Brain Ischemia] explode all trees \\
\hline$\# 5$ & MeSH descriptor: [Carotid Artery Diseases] explode all trees \\
\hline$\# 6$ & MeSH descriptor: [Cerebral Small Vessel Diseases] explode all trees \\
\hline$\# 7$ & MeSH descriptor: [Intracranial Arterial Diseases] explode all trees \\
\hline$\# 8$ & MeSH descriptor: [Intracranial Embolism and Thrombosis] explode all trees \\
\hline$\# 9$ & MeSH descriptor: [Intracranial Hemorrhages] explode all trees \\
\hline$\# 10$ & MeSH descriptor: [Stroke] this term only \\
\hline$\# 11$ & MeSH descriptor: [Brain Infarction] explode all trees \\
\hline
\end{tabular}




\#12 MeSH descriptor: [Vasospasm, Intracranial] this term only

\begin{tabular}{ll}
\hline$\# 13$ & MeSH descriptor: [Vertebral Artery Dissection] this term only \\
\hline$\# 14$ & (stroke* or poststroke or apoplex* or cerebral vasc ${ }^{\star}$ or brain vasc ${ }^{\star}$ or cerebrovasc ${ }^{\star}$ or cva $^{\star}$ or $^{*}$ \\
& SAH):ti,ab,kw (Word variations have been searched)
\end{tabular}

((brain* or cerebr ${ }^{\star}$ or cerebell* or vertebrobasil ${ }^{\star}$ or hemispher ${ }^{\star}$ or intracran * or intracerebral or infratentorial or supratentorial or middle cerebral artery or MCA* or anterior circulation or posterior

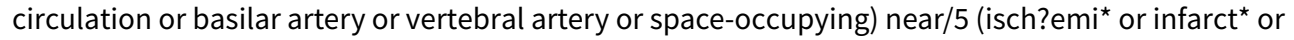
thrombo* or emboli* or occlus* or hypoxi*)):ti,ab,kw (Word variations have been searched)

$\begin{array}{ll}\text { \#16 } & \left(\left(\text { brain }^{\star} \text { or cerebr* or cerebell* or intracerebral or intracran* or parenchymal or intraparenchy- }\right.\right. \\ \text { mal or intraventricular or infratentorial or supratentorial or basal gangli* or putaminal or puta- } \\ \text { men or posterior fossa or hemispher or subarachnoid) near/5 (h?emorrhag* or h?ematoma* or } \\ \left.\left.\text { bleed }{ }^{\star}\right)\right) \text {.tw.:ti,ab,kw (Word variations have been searched) }\end{array}$

\begin{tabular}{|c|c|}
\hline \#17 & MeSH descriptor: [Hemiplegia] this term only \\
\hline \#18 & MeSH descriptor: [Paresis] explode all trees \\
\hline \#19 & MeSH descriptor: [Gait Disorders, Neurologic] explode all trees \\
\hline \#20 & $\begin{array}{l}\text { (hemipleg* or hemipar }{ }^{\star} \text { or paresis or paraparesis or paretic):ti,ab,kw (Word variations have been } \\
\text { searched) }\end{array}$ \\
\hline \#21 & $\{$ or \#1-\#20\} \\
\hline \#22 & MeSH descriptor: [Electric Stimulation] explode all trees \\
\hline \#23 & MeSH descriptor: [Electric Stimulation Therapy] this term only \\
\hline \#24 & MeSH descriptor: [Electrodes] this term only \\
\hline \#25 & MeSH descriptor: [Electrodes, Implanted] this term only \\
\hline \#26 & MeSH descriptor: [Implantable Neurostimulators] explode all trees \\
\hline \#27 & MeSH descriptor: [Ion-Selective Electrodes] this term only \\
\hline \#28 & MeSH descriptor: [Microelectrodes] this term only \\
\hline \#29 & MeSH descriptor: [Signal Processing, Computer-Assisted] this term only \\
\hline \#30 & MeSH descriptor: [Man-Machine Systems] this term only \\
\hline \#31 & MeSH descriptor: [User-Computer Interface] this term only \\
\hline \#32 & MeSH descriptor: [Electromyography] this term only \\
\hline \#33 & (neuroprosthes* or neuroprosthetic ${ }^{\star}$ ):ti,ab,kw (Word variations have been searched) \\
\hline \#34 & 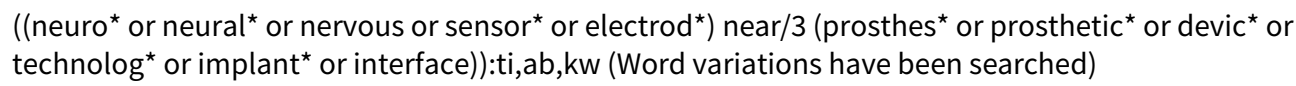 \\
\hline
\end{tabular}


\#36 ((electric ${ }^{\star}$ or nerv* or neuro*) near/3 stimul $\left.{ }^{\star}\right):$ ti,ab,kw (Word variations have been searched)

\begin{tabular}{|c|c|}
\hline \#37 & ((electromyography or emg) near/3 trigger $\left.^{\star}\right): \mathrm{ti}, \mathrm{ab}, \mathrm{kw}$ (Word variations have been searched) \\
\hline \#38 & (foot drop near/3 stimulat*):ti,ab,kw (Word variations have been searched) \\
\hline \#39 & $\{$ or \#22-\#38\} \\
\hline$\# 40$ & $\# 21$ and \#39 \\
\hline
\end{tabular}

\section{Search results: 2554}

\section{Appendix 2. MEDLINE search strategy}

MEDLINE (Ovid) search strategy (from 1946)

1. cerebrovascular disorders/ or exp basal ganglia cerebrovascular disease/ or exp brain ischemia/ or exp carotid artery diseases/ or exp cerebral small vessel diseases/ or exp intracranial arterial diseases/ or exp "intracranial embolism and thrombosis"/ or exp intracranial hemorrhages/ or stroke/ or exp brain infarction/ or stroke, lacunar/ or vasospasm, intracranial/ or vertebral artery dissection/

2. (stroke\$ or poststroke or apoplex\$ or cerebral vasc\$ or brain vasc\$ or cerebrovasc\$ or cva $\$$ or SAH).tw.

3. ((brain\$ or cerebr\$ or cerebell\$ or vertebrobasil\$ or hemispher\$ or intracran\$ or intracerebral or infratentorial or supratentorial or middle cerebral artery or MCA $\$$ or anterior circulation or posterior circulation or basilar artery or vertebral artery or space-occupying) adj5 (isch? emi\$ or infarct\$ or thrombo\$ or emboli\$ or occlus\$ or hypoxi\$)).tw.

4. ((brain\$ or cerebr\$ or cerebell\$ or intracerebral or intracran\$ or parenchymal or intraparenchymal or intraventricular or infratentorial or supratentorial or basal gangli\$ or putaminal or putamen or posterior fossa or hemispher\$ or subarachnoid) adj5 (h?emorrhag\$ or h? ematoma\$ or bleed\$)).tw.

5. hemiplegia/ or exp paresis/ or exp Gait Disorders, Neurologic/

6. (hemipleg\$ or hemipar\$ or paresis or paraparesis or paretic).tw.

7. or/1-6

8. exp Electric Stimulation/

9. Electric Stimulation Therapy/

10. electrodes/ or electrodes, implanted/ or exp implantable neurostimulators/ or ion-selective electrodes/or microelectrodes/

11. Signal Processing, Computer-Assisted/

12. man-machine systems/ or user-computer interface/

13. electromyography/

14. (neuroprosthes\$ or neuroprosthetic\$).tw.

15. ((neuro\$ or neural\$ or nervous or sensor\$ or electrod\$) adj3 (prosthes\$ or prosthetic\$ or devic\$ or technolog\$ or implant\$ or interface)).tw.

16. (neurostim\$ or electroneurostim\$ or electrostim\$).tw.

17. ((electric\$ or nerv\$ or neuro\$) adj3 stimul\$).tw.

18. ((electromyography or emg) adj3 trigger\$).tw.

19. (foot drop adj3 stimulat\$).tw.

20. or/8-19 
21. randomized controlled trial.pt.

22. controlled clinical trial.pt.

23. randomized.ab.

24. placebo.ab.

25. randomly.ab.

26. trial.ab.

27. groups.ab.

28. or $/ 21-27$

29. 7 and 20 and 28

Search results: 2512

\section{Appendix 3. Embase Ovid search strategy}

1. cerebrovascular disease/ or brain disease/ or exp basal ganglion hemorrhage/ or exp brain hemangioma/ or exp brain hematoma/ or exp brain hemorrhage/ or exp brain infarction/ or exp brain ischemia/ or exp carotid artery disease/ or exp cerebral artery disease/ or exp cerebrovascular accident/ or exp cerebrovascular malformation/ or exp intracranial aneurysm/ or exp occlusive cerebrovascular disease/ or exp vertebrobasilar insufficiency/

2. (stroke\$ or poststroke or apoplex\$ or cerebral vasc\$ or brain vasc\$ or cerebrovasc\$ or cva $\$$ or $\mathrm{SAH}) . \mathrm{tw}$.

3. ((brain or cerebr\$ or cerebell\$ or vertebrobasil\$ or hemispher\$ or intracran\$ or intracerebral or infratentorial or supratentorial or middle cerebral artery or MCA or anterior circulation or posterior circulation or basilar artery or vertebral artery or space-occupying) adj5 (isch? emi\$ or infarct\$ or thrombo or emboli\$ or occlus\$ or hypoxi\$)).tw.

4. ((brain\$ or cerebr\$ or cerebell\$ or intracerebral or intracran\$ or parenchymal or intraparenchymal or intraventricular or infratentorial or supratentorial or basal gangli\$ or putaminal or putamen or posterior fossa or hemispher\$ or subarachnoid) adj5 (h?emorrhag\$ or h? ematoma\$ or bleed\$)).tw.

5. exp hemiplegia/ or exp paresis/ or neurologic gait disorder/

6. (hemipleg\$ or hemipar\$ or paresis or paraparesis or paretic).tw.

7. or/1-6

8. electrotherapy/ or exp high frequency electrotherapy/ or exp low frequency electrotherapy/

9. electrostimulation/

10. electrode/ or cortical electrode/ or electromyograph electrode/ or microelectrode/ or ion selective electrode/

11. implantable neurostimulator/ or neurological implant/

12. motor neuroprosthesis/ or neuroprosthesis/ or "neurological prosthesis and implant"/

13. electromyograph electrode/ or electromyograph/ or electromyography/

14. (neuroprosthes\$ or neuroprosthetic\$).tw.

15. ((neuro\$ or neural\$ or nervous or sensor\$ or electrod\$) adj3 (prosthes\$ or prosthetic\$ or devic\$ or technolog\$ or implant\$ or interface)).tw.

16. (neurostim\$ or electroneurostim\$ or electrostim\$).tw.

17. ((electric\$ or nerv\$ or neuro\$) adj3 stimul\$).tw.

18. ((electromyography or emg) adj3 trigger\$).tw.

19. (foot drop adj3 stimulat\$).tw. 
20. or/8-19

21. Randomized Controlled Trial/ or "randomized controlled trial (topic)"/

22. Randomization/

23. Controlled clinical trial/ or "controlled clinical trial (topic)"/

24. control group/ or controlled study/

25. clinical trial/ or "clinical trial (topic)"/ or phase 1 clinical trial/ or phase 2 clinical trial/ or phase $3 \mathrm{clinical}$ trial/ or phase $4 \mathrm{clinical}$ trial/

26. Crossover Procedure/

27. Double Blind Procedure/

28. Single Blind Procedure/ or triple blind procedure/

29. placebo/ or placebo effect/

30. (random\$ or RCT or RCTs).tw.

31. (controlled adj5 (trial\$ or stud\$)).tw.

32. (clinical\$ adj5 trial\$).tw.

33. ((control or treatment or experiment\$ or intervention) adj5 (group\$ or subject\$ or patient\$)).tw.

34. (quasi-random\$ or quasi random\$ or pseudo-random\$ or pseudo random\$).tw.

35. ((control or experiment\$ or conservative) adj5 (treatment or therapy or procedure or manage\$)).tw.

36. ((singl\$ or doubl\$ or tripl\$ or trebl\$) adj5 (blind\$ or mask\$)).tw.

37. (cross-over or cross over or crossover).tw.

38. (placebo $\$$ or sham).tw.

39. trial.ti.

40. (assign\$ or allocat\$).tw.

41. controls.tw.

42. or/21-41

43. 7 and 20 and 42

Search results: 6460

\section{Appendix 4. CINAHL EBSCO search strategy}

S1 (MH "Cerebrovascular Disorders") OR (MH "Basal Ganglia Cerebrovascular Disease+") OR (MH "Carotid Artery Diseases+") OR (MH "Cerebral Ischemia+") OR (MH "Cerebral Vasospasm") OR (MH "Intracranial Arterial Diseases+") OR ( (MH "Intracranial Embolism and Thrombosis") ) OR (MH "Intracranial Hemorrhage+") OR (MH "Stroke") OR (MH "Vertebral Artery Dissections") OR (MH "Stroke Patients") OR (MH "Stroke Units")

S2 $\mathrm{TI}$ ( stroke or poststroke or post-stroke or cerebrovasc* or brain vasc* or cerebral vasc or cva or apoplex or SAH ) or AB ( stroke or poststroke or post-stroke or cerebrovasc* or brain vasc* or cerebral vasc or cva or apoplex or SAH)

S3 TI ((brain or cerebr ${ }^{\star}$ or cerebell ${ }^{*}$ or vertebrobasil ${ }^{*}$ or hemispher ${ }^{\star}$ or intracran* or intracerebral or infratentorial or supratentorial or middle cerebral artery or $\mathrm{MCA}^{\star}$ or anterior circulation or posterior circulation or basilar artery or vertebral artery or space-occupying) N5 ( ischemi ${ }^{\star}$ or ischaemi ${ }^{\star}$ or infarct ${ }^{\star}$ or thrombo* or emboli* or occlus $\left.{ }^{\star}\right)$ ) OR AB ((brain or cerebr ${ }^{\star}$ or cerebell $^{\star}$ or vertebrobasil ${ }^{\star}$ or hemispher ${ }^{\star}$ or intracran ${ }^{\star}$ or intracerebral or infratentorial or supratentorial or middle cerebral artery or MCA* or anterior circulation or posterior circulation or basilar artery or vertebral artery or space-occupying) N5 (ischemi* or ischaemi* or infarct* or thrombo* or emboli* or occlus*))

S4 TI (( brain* or cerebr ${ }^{\star}$ or cerebell* or intracerebral or intracran* or parenchymal or intraparenchymal or intraventricular or infratentorial or supratentorial or basal gangli* or putaminal or putamen or posterior fossa or hemispher or subarachnoid ) N5 ( haemorrhage* or hemorrhage ${ }^{\star}$ or haematoma* or hematoma $^{\star}$ or bleed $\left.\left.^{\star}\right)\right)$ OR AB (( brain ${ }^{\star}$ or cerebr ${ }^{\star}$ or cerebell ${ }^{\star}$ or intracerebral or intracran ${ }^{\star}$ or parenchymal 
or intraparenchymal or intraventricular or infratentorial or supratentorial or basal gangli* or putaminal or putamen or posterior fossa or hemispher* or subarachnoid ) N5 ( haemorrhage* or hemorrhage* or haematoma* or hematoma* or bleed*))

S5 (MH "Hemiplegia") or (MH "Gait Disorders, Neurologic+")

$\mathrm{S} 6 \mathrm{TI}$ (hemipleg* or hemipar* or paresis or paretic) OR AB (hemipleg* or hemipar* or paresis or paretic)

S7 (MH "Brain Injuries") OR (MH "Brain Damage, Chronic") OR (MH "Brain Concussion+") OR (MH "Head Injuries") OR (MH "Brain Abscess+") S8 TI ( ((brain or head or intracran* or cerebr* or cerebell* or orbit ${ }^{\star}$ or brainstem or vertebrobasil*) N5 (abscess ${ }^{\star}$ or injur $^{\star}$ or contusion* $^{\star}$ or hypoxi* or damage* or inflamm* or concussion or trauma* or fractur ${ }^{\star}$ or infection* or lesion*)) ) OR AB ( ((brain or head or intracran* or cerebr ${ }^{\star}$ or cerebell* or orbit* or brainstem or vertebrobasil ${ }^{\star}$ ) N5 (abscess ${ }^{\star}$ or injur ${ }^{\star}$ or contusion* or hypoxi* or damage* or inflamm ${ }^{\star}$ or concussion or trauma* or fractur ${ }^{\star}$ or infection* or lesion*)) )

S9 S1 OR S2 OR S3 OR S4 OR S5 OR S6 OR S7 OR S8

S10 (MH "Lower Extremity+")

S11 (MH "Tarsal Joint+") OR (MH "Toe Joint+") OR (MH "Ankle Joint") OR (MH "Knee Joint+")

S12 TI ( (lower extremit* or leg or legs or ankle* or foot or feet or heel ${ }^{\star}$ or toe* or hip or knee or knees or thigh*) ) OR AB ( (lower extremit* or leg or legs or ankle* or foot or feet or heel* or toe* or hip or knee or knees or thigh*))

S13 TI ( (walk* or gait* or ambulat* or mobil* or locomot* or balanc ${ }^{\star}$ or stride or foot-drop) ) OR AB ( (walk* or gait* or ambulat* or mobil* or locomot* or balanc* or stride or foot-drop))

S14 (MH "Locomotion+")

S15 S10 OR S11 OR S12 OR S13 OR S14

S16 (MH "Guided Imagery") OR (MH "Imagination") OR (MH "Mirror Therapy") OR (MH "Reflection")

S17 (MH "Mental Processes") OR (MH "Perception+")

S18 (MH "Imitative Behavior")

S19 (MH "Psychomotor Performance+")

S20 TI ( ((motor or locomot $\left.{ }^{\star}\right)$ N3 (imag* or visual ${ }^{\star}$ or ideation $\left.)\right)$ ) OR AB ( ((motor or locomot $\left.{ }^{\star}\right)$ N3 (imag* or visual ${ }^{\star}$ or ideation)) )

S21 TI ( (action N3 (immitat* or observ* or visuali* or ideation)) ) OR AB ( (action N3 (immitat ${ }^{\star}$ or observ* or visuali* or ideation)) )

S22 TI ( ((cognitive or covert ${ }^{\star}$ or mental) N3 (practic ${ }^{\star}$ or rehears ${ }^{\star}$ or represent ${ }^{\star}$ or visual ${ }^{\star}$ or image $)$ ) ) OR AB ( ((cognitive or covert* or mental) N3 (practic ${ }^{\star}$ or rehears ${ }^{\star}$ or represent ${ }^{\star}$ or visual ${ }^{\star}$ or image $\left.\left.{ }^{\star}\right)\right)$ )

S23 TI ( ((visual or mirror $\left.{ }^{\star}\right)$ N3 (reflection or illusion or feedback or therapy)). ) OR AB ( ((visual or mirror ${ }^{\star}$ ) N3 (reflection or illusion or feedback or therapy)). )

S24 S16 OR S17 OR S18 OR S19 OR S20 OR S21 OR S22 OR S23

S25 MH Random Assignment or MH Single-blind Studies or MH Double-blind Studies or MH Triple-blind Studies or MH Crossover design or MH Factorial Design

S26 TI ("multicentre study" or "multicenter study" or "multi-centre study" or "multi-center study") or AB ("multicentre study" or "multicenter study" or "multi-centre study" or "multi-center study") or SU ("multicentre study" or "multicenter study" or "multi-centre study" or "multi-center study")

S27 TI random* or AB random*

S28 AB "latin square" or TI "latin square"

S29 TI (crossover or cross-over) or AB (crossover or cross-over) or SU (crossover or cross-over)

S30 MH Placebos

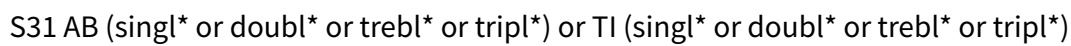

S32 TI blind ${ }^{\star}$ or AB mask* or AB blind* or TI mask*

S33 S31 and S32

S34 TI Placebo* or AB Placebo* or SU Placebo*

S35 MH Clinical Trials

S36 TI (Clinical AND Trial) or AB (Clinical AND Trial) or SU (Clinical AND Trial)

$\mathrm{S} 37 \mathrm{~S} 25$ or S26 or S27 or S28 or S29 or S30 or S33 or S34 or S35 or S36

S38 S9 AND S15 AND S24 AND S37

\section{Search results: 782}

\section{Appendix 5. AMED Ovid search strategy}

1. cerebrovascular disorders/ or cerebral hemorrhage/ or cerebral infarction/ or cerebral ischemia/ or cerebrovascular accident/

2. (stroke\$ or poststroke or apoplex\$ or cerebral vasc\$ or brain vasc\$ or cerebrovasc\$ or cva\$ or SAH).tw.

3. ((brain\$ or cerebr\$ or cerebell\$ or vertebrobasil\$ or hemispher\$ or intracran\$ or intracerebral or infratentorial or supratentorial or middle cerebral artery or MCA $\$$ or anterior circulation or posterior circulation or basilar artery or vertebral artery or space-occupying) adj5 (isch? emi\$ or infarct\$ or thrombo\$ or emboli\$ or occlus\$ or hypoxi\$)).tw.

4. ((brain\$ or cerebr\$ or cerebell\$ or intracerebral or intracran\$ or parenchymal or intraparenchymal or intraventricular or infratentorial or supratentorial or basal gangli\$ or putaminal or putamen or posterior fossa or hemispher\$ or subarachnoid) adj5 (h?emorrhag\$ or h? ematoma\$ or bleed\$)).tw. 
5. hemiplegia/

6. gait disorders/

7. (hemipleg\$ or hemipar\$ or paresis or paraparesis or paretic).tw.

8.1 or 2 or 3 or 4 or 5 or 6 or 7

9. electric stimulation/

10. electrotherapy/ or functional electric stimulation/

11. electrodes/ or prosthesis/ or prosthesis design/

12. electromyography/

13. (neuroprosthes\$ or neuroprosthetic\$).tw.

14. ((neuro\$ or neural\$ or nervous or sensor\$ or electrod\$) adj3 (prosthes\$ or prosthetic\$ or devic\$ or technolog\$ or implant\$ or interface)).tw.

15. (neurostim\$ or electroneurostim\$ or electrostim\$).tw.

16. ((electric\$ or nerv\$ or neuro\$) adj3 stimul\$).tw.

17. ((electromyography or emg) adj3 trigger\$).tw.

18. (foot drop adj3 stimulat\$).tw.

19.9 or 10 or 11 or 12 or 13 or 14 or 15 or 16 or 17 or 18

20. clinical trials/

21. randomized controlled trials/

22. comparative study/

23. double blind method/

24. random allocation/

25. placebos/

26. random\$.tw.

27. (controlled adj5 (trial\$ or stud\$)).tw.

28. (clinical\$ adj5 trial\$).tw.

29. placebo\$.tw.

30. controls.tw.

31.20 or 21 or 22 or 23 or 24 or 25 or 26 or 27 or 28 or 29 or 30

32. 8 and 19 and 31

Search results: 267

Appendix 6. PEDro (Physiotherapy Evidence Database) search strategy

"Therapy": electrotherapies, heat, cold

"Problem": motor incoordination

"Subdiscipline": neurology

"Method": clinial trial 


\section{Search results: 667}

\section{Appendix 7. REHABDATA search strategy}

1. '"neur* AND orthos ${ }^{\star \prime \prime \prime}$

2. '"neur AND prosthes*"'

3. '"neuroprosthes*"'

Search results: 29

\section{Appendix 8. IEEE search strategy}

"Document Title": stroke OR cerebrovascular or cerebral OR intracerebral OR intracranial

(("Document Title":neuroprosthesis OR neurostimulation OR neurostim* OR electroneurostim* OR electrostim*) AND "Document Title":stroke OR cerebrovascular)

\section{Search results: 585}

\section{Appendix 9. US National Institutes of Health Ongoing Trials Register ClinicalTrials.gov search strategy}

( neuroprosthesis OR neuroprosthetic OR "functional electrical stimulation") AND ( Brain Infarction OR Intracranial Hemorrhages OR Carotid Artery Diseases OR Brain Ischemia OR Cerebral Hemorrhage OR Cerebrovascular Disorders OR Stroke ) [DISEASE]

\section{Search results: 88}

\section{Appendix 10. World Health Organization (WHO) International Clinical Trials Registry Platform search strategy}

stroke AND neuroprosthesis OR stroke AND neuroprosthetic OR stroke AND functional electrical stimulation OR stroke AND electroneurostimulation OR stroke AND electrostimulation

cerebrovascular AND neuroprosthesis OR cerebrovascular AND neuroprosthetic OR cerebrovascular AND functional electrical stimulation OR cerebrovascular AND electroneurostimulation OR cerebrovascular AND electrostimulation

\section{Search results: 113}

\section{Appendix 11. Stroke Trials Registry search strategy}

"Interventions":

Neuroprosthesis

Functional Electrical Stimulation

Functional electrical stimulation (FES)

Functional Electrical Stimulation (FES) through the Ness H200

Functional Electrical Stimulation (FES) treatment

Functional Electric Stimulation

Functional electrical stimulator

Functional Neuromotor Stimulation

Neuromuscular electrical stimulator

Neuromuscular electrical stimulation (NMES)

Surface Functional Electrical Stimulation

Surface functional electrical stimulation (FES) assisted moviment training

Surface Functional Neuromuscular Stimulation

Self-designed surface functional electrical stimulator 
Electrical stimulation

Electrical stimulation with exercises

Electrical Stimulation; Rehabilitation Robot

Electrical stimulator

Electrically Assisted Movement Therapy

Intramuscular Electrical Stimulator

electrodes

Walking with ankle electrical stimulation

Contralaterally Controlled Neuromuscular Electrical Stimulation

Electrical Stimulation with Intramuscular Electrodes

Functional Neuromuscular stimulation with intramuscular electrodes

Smart glove system with functional electrical stimulation

Electrical stimulation-dynamic hand orthosis

Myoelectric-Elbow-Wrist-Hand orthosis

Electrical stimulation-dynamic hand orthosis

DC-stimulation (Neuroconn, Germany)

Neuromodulation electroencephalographic signals and functional electrical stimulation

Cortical Electrical Stimulation

Implantation of NeuroPort Arrays in motor cortex

$\mathrm{BCl}$-controlled neurorehabilitation device

Robot-assisted neurocognitive therapy of hand function

\section{Search results: 54}

\section{Appendix 12. ISRCTN registry search strategy}

"Condition": Stroke

\section{Search results: 377}

\section{Appendix 13. Australian New Zealand Clinical Trials Registry search strategy}

"Registry": ANZCTR

"Intervention code": Treatment: Devices OR Treatment: Other OR Rehabilitation OR Other interventions

"Study type": Interventional

"Allocation to intervention": Randomised

"Health Condition(s) or problem(s) studied": Stroke

"Condition category": Neurological

"Age group": Adult (18yrs and over)

Search results: 31 
Appendix 14. Health Technology Assessment (HTA) database - Centre for Reviews and Dissemination, University of York search strategy

1. MeSH DESCRIPTOR Cerebrovascular Disorders EXPLODE ALL TREES IN HTA

Search results: 55

Appendix 15. OAlster search strategy

neuroprosthes*

neuro prosthes*

Search results: 98

Appendix 16. The Directory of Open Access Repositories - OpenDOAR search strategy

Searched using CORE

title:((neuroprosthes $\left.\left.{ }^{\star}\right)\right)$ abstract:((neuroprosthes $\left.\left.{ }^{\star}\right)\right)$

Search results: 37

\section{Appendix 17. British Library Ethos search strategy}

Stroke and "electrical stimulation"

Search results: 30

\section{Appendix 18. ProQuest Dissertations \& Theses Global search strategy}

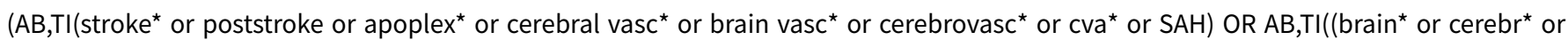
cerebell $^{\star}$ or vertebrobasil ${ }^{\star}$ or hemispher ${ }^{\star}$ or intracran* or intracerebral or infratentorial or supratentorial or middle cerebral artery or MCA* or "anterior circulation" or "posterior circulation" or basilar artery or vertebral artery or space-occupying) AND (ischaemi* or ischemi* or infarct* or thrombo* or emboli* or occlus* or hypoxi $\left.{ }^{\star}\right)$ ) OR AB,TI((brain* or cerebr* or cerebell* or intracerebral or intracran* or parenchymal or intraparenchymal or intraventricular or infratentorial or supratentorial or basal gangli* or putaminal or putamen or "posterior fossa" or hemispher ${ }^{\star}$ or subarachnoid) AND (haemorrhag* or hemorrhag* or haematoma* or hematoma or bleed $\left.{ }^{\star}\right)$ )) AND (AB,TI(neuroprosthes* or neuroprosthetic $\left.{ }^{\star}\right)$ OR AB,TI((neuro* or neural ${ }^{\star}$ or nervous or sensor ${ }^{\star}$ or electrod $\left.{ }^{\star}\right)$ AND (orthos or orthotic $^{\star}$ or prosthes ${ }^{\star}$ or prosthetic or $^{\star}$ devic $^{\star}$ or technolog* or implant* or implant* or interface)) OR AB,TI(neurostim* or electroneurostim* or electrostim $\left.{ }^{\star}\right)$ OR AB,TI((electric* or nerv* or neuro*) AND stimul*) OR AB,TI(foot drop AND stimulat*))

Search results: 1292

\section{Appendix 19. Email sent to equipment manufacturers}

My name is Luciana Mendes. I am currently undertaking a Cochrane Review that focuses on motor neuroprosthesis directed to upper or lower limb for improving activities and participation in people after stroke ('Motor neuroprosthesis for recovery of function after stroke' available at https://www.cochranelibrary.com/cdsr/doi/10.1002/14651858.CD012991/full ).

This way, I contact this company to know if you developed or sponsored randomised controlled trial (published or unpublished) that uses motor neuroprosthesis devices as an orthosis in home or community context. If so, let me know that I will assess the study and examine the possibility of inclusion of it in the review.

If you have any questions let me know. I am available for any clarification regarding the review.

\section{Appendix 20. The Canadian Agency for Drugs and Technologies in Health - CADTH search strategy}

Health Technology Assessment (HTA) Agencies:

1. Alberta Health and Wellness

Keyword: Stroke

2. Canadian Agency for Drugs and Technologies in Health (CADTH)

Keyword: Stroke

3. Drug Safety and Effectiveness Network (DSEN)

Filter items: electrical 
4. Health Quality Council of Alberta (HQCA)

Keyword: "electrical"

5. Health Quality Ontario (HQO)

Search: "electrical stimulation"

6. Institut national d'excellence en santé et en services sociaux (INESSS)

Keyword: accident vasculaire cerebral

7. Institute of Health Economics (IHE)

Keyword: stroke

8. McGill University Health Centre (MUHC). Technology Assessment Unit Reports

Search: "electrical stimulation"

9. NLCAHR: Newfoundland and Labrador Centre for Applied Health Research. Contextualized Health Research Synthesis Program (CHRSP) Completed CHRSP projects

Ongoing projects at "Current CHRSP Projects" link

10. Ottawa Hospital Research Institute (OHRI) Knowledge Synthesis Group

11. Programs for assessment of Technology in Health (Canada) Reports (PATH)

12. Therapeutics Initiative. Therapeutics Letter

13. INAHTA Secretariat. International Network of Agencies for Health Technology Assessment (INAHTA)

Keywords: electrical stimulation

14. World Health Organization Regional Office for Europe. Health Evidence Network (WHO HEN)

Keyword: electrical stimulation

15. Australian Government. Department of Health and Ageing. Australia and New Zeland Horizon Scanning Network (ANZHSN)

Keyword: electrical stimulation

16. Australian Government Department of Health and Ageing. Medical Services Advisory Committee (MSAC). MSAC Applications Search: "electrical stimulation"

17. Joanna Briggs Institute (JBI) JBI EBP Database

Search: "electrical stimulation" (Title, Abstract or Keywords) AND stroke (Title, Abstract or Keywords)

18. Queensland Government (Australia). Health Technology Reference Group. Health Technologies Evaluated-Reports and Briefs (COAG Health Council)

Search: "electrical stimulation"

19. Kenniscentrum voor de Gezondheidszorg / Le Centre d'expertise des soins de santé. Belgian Health Care Knowledge Centre (KCE) Topic: Neurology and brain disease

20. Haute Autorité de santé/ French National Authority for Health (HAS). Haute Autorité de santé

Topic: Prostheses and implants

21. Health Information and Quality Authority. Health Technology Assessments

Keywords: electrical

22. Health Service Executive. Irish Health Repository (Lenus)

Motor neuroprosthesis for promoting recovery of function after stroke (Review) 
Search: "electrical stimulation"

23. Zorginstituut Nederland. National Health Care Institute Netherlands

Keywords: stimulation

24. Nasjonalt kunnskapssenter for helsetjenesten. Norwegian Knowledge Centre for the Health Services.Publications

Keywords: stimulation

25. Swedish Council on Health Technology Assessment (SBU).

Search: stimulation

26. Healthcare Improvement Scotland. Published Resources

Search: electrical

27. National Institute for Health and Care Excellence (NICE). NHS National Institute for Health and Care Excellence

Search: electrical

28. National Institute for Health Research. (NIHR).Innovation Observatory

Search: neuroprosthesis

29. NHS Purchasing and Supply Agency. Centre for Evidence-based Purchasing (CEP)

Search: electrical [All Report Types]

30. NIHR Evaluation, Trials and Studies Coordinating Centre (NETSCC). Research Project

Keywords: "electrical stimulation"

31. National Health Service UK (NHS). NHS England

Keywords: "electrical stimulation"

32. Washington State Health Care Authority (HCA). Health Technology Review

Search: neuroprosthesis

Databases (FREE)

\section{LILACS}

Search: stroke AND stimulation

2. McMaster University, McMaster Health Forum. Health Systems Evidence

Search: stroke AND stimulation

3. TRIP Database (TRIP). Trip Database - Clinical Search Engine

Search: neuroprosthesis

\section{CONTRIBUTIONSOF AUTHORS}

Luciana Mendes: conceived the review question; developed, completed, and edited the first draft of the protocol; drafted the final protocol; and made an intellectual contribution to the protocol. She searched some electronic databases with the help of the Information Specialist, screened titles and abstracts of publications identified by the search, selected and assessed trials, extracted trial and outcome data, contacted trialists about unpublished data, assessed the methodological quality of selected trials, carried out statistical analysis and interpretation of the data, drafted the review, and approved the final manuscript of the review.

íllia Lima: developed and completed part of the first draft of the protocol and made an intellectual contribution to the protocol. Together with Luciana Mendes she screened titles and abstracts of publications identified by the search and selected and assessed trials; she also checked the outcome data extracted by Luciana Mendes. 
Túlio Souza: contributed with clinical expertise, advised on and developed the protocol, and made an intellectual contribution to the protocol. He advised in case of disagreement on the selection of studies, data extraction, and assessment of risk of bias; contributed to the interpretation of the data; and approved the final manuscript of the review.

George Nascimento: contributed with clinical expertise on devices, advised on and developed the protocol, made an intellectual contribution to the protocol, and approved the final version prior to submission. He contributed to the interpretation of the data and approved the final manuscript of the review.

Vanessa Resqueti: advised on and developed the protocol, participated as an arbiter, and made an intellectual contribution to the protocol. She advised in case of disagreement on the selection of studies, data extraction, and assessment of risk of bias; contributed to the interpretation of the data; and approved the final manuscript of the review.

Guilherme Fregonezi: developed and co-ordinated the protocol, secured funding, advised on and made an intellectual contribution to the protocol, and approved the final version prior to submission. He interpreted the data and the analysis, and corrected and approved the final manuscript of the review.

\section{DECLARATIONS OF INTEREST}

Luciana Mendes: none known

íllia Lima: none known

Túlio Souza: none known

George Nascimento: none known

Vanessa Resqueti: none known

Guilherme Fregonezi: none known

\section{SOURCES OF SUPPORT}

\section{Internal sources}

- Federal University of Rio Grande do Norte, Brazil.

\section{External sources}

- No sources of support supplied

\section{DIFFERENCES BETWEEN PROTOCOL AND REVIEW}

We used Covidence software for the selection of studies, data extraction, and assessment of risk of bias (Covidence). We included another review author (TS) to help the third review author (VR) in the evaluation of discrepancies and providing advice in case of disagreement on the selection of studies, data extraction, and assessment of risk of bias.

We conducted an extensive search, and are therefore confident that we have identified all relevant studies in the field. However, we did not use Science Citation Index Cited Reference Search for forward tracking of important articles. Due to technical problems with OpenDOAR repository, we used CORE for this repository content search.

We only identified individually randomized trials for this review, so we did not need to analyze for unit of analysis issues as planned in our protocol (Mendes 2018).

Our protocol prespecified a number of subgroup analyses including type of effect and duration of use of device. However, as we analyzed the outcome data only as endpoint values (and not changes from baseline), we decided not to perform a subgroup analysis for type of effect. Regarding the subgroup analysis duration of use of device, we decided to define some primary outcomes based on different periods (such as walking speed up to six months of device use, walking speed between six and 12 months of device use) instead of carrying out the proposed subgroup analysis. This change was based on the fact that we could gain a better understanding of the effect of $\mathrm{MN}$ on different periods of use without unit of analysis error (Higgins 2011c), considering that studies could have repeated observations on participants for the same study. We did not perform subgroup analysis for the effect of $\mathrm{MN}$ when applied to lower limb or upper limb or for the effect of MN when used by participants in different phases of stroke because there were no data available for MN applied to upper limb, and there were no data for participants less than three months since stroke onset. 


\section{N D EX TERMS}

\section{Medical Subject Headings (MeSH)}

Activities of Daily Living; Electric Stimulation Therapy [ ${ }^{\star}$ methods]; Motor Activity [physiology]; Quality of Life; Randomized Controlled Trials as Topic; Recovery of Function; Stroke [therapy]; Stroke Rehabilitation [ ${ }^{\star}$ methods]

\section{MeSH check words}

Humans 